\title{
JASN
}

\section{Prevalence Estimates of Predicted Pathogenic COL4A3 - COL4A5 Variants in a Population Sequencing Database and Their Implications for Alport Syndrome}

\begin{tabular}{|c|c|}
\hline Journal: & Journal of the American Society of Nephrology \\
\hline Manuscript ID & JASN-2020-07-1065.R3 \\
\hline Manuscript Type: & Original Article - Basic Research \\
\hline $\begin{array}{r}\text { Date Submitted by the } \\
\text { Author: }\end{array}$ & 29-Apr-2021 \\
\hline Complete List of Authors: & $\begin{array}{l}\text { Gibson, Joel; The University of Melbourne Department of Medicine Austin } \\
\text { Health and Northern Health } \\
\text { Fieldhouse, Rachel; Garvan Institute of Medical Research, Kinghorn } \\
\text { Centre for Clinical Genomics } \\
\text { Chan, Melanie; University College London, Department of Renal } \\
\text { Medicine; Queen Mary University of London, Genomics England } \\
\text { Sadeghi-Alavijeh, Omid; University College London, Department of Renal } \\
\text { Medicine; Queen Mary University of London, Genomics England } \\
\text { Burnett, Leslie; Garvan Institute of Medical Research, Kinghorn Centre } \\
\text { for Clinical Genomics } \\
\text { Izzi, Valerio; University of Oulu, Center for Cell-Matrix Research and } \\
\text { Biocenter Oulu } \\
\text { Persikov, Anton; Princeton University Princeton Center for Quantitative } \\
\text { Biology Lewis-Sigler Institute for Integrative Genomics } \\
\text { Gale, Daniel; University College London, Department of Renal Medicine; } \\
\text { Queen Mary University of London, Genomics England } \\
\text { Storey, Helen; Guy's Hospital, Molecular Genetics, Viapath Laboratories } \\
\text { Savige, Judy; The University of Melbourne Department of Medicine } \\
\text { Austin Health and Northern Health }\end{array}$ \\
\hline Keywords: & $\begin{array}{l}\text { Alport-s syndrome, Thin basement membrane nephropathy, Haematuria, } \\
\text { COL4A5, COL4A3, COL4A4, collagen IV, genetic renal disease, } \\
\text { glomerular basement membrane, Gly substitutions }\end{array}$ \\
\hline
\end{tabular}

\section{SCHOLARONE Manuscripts}


Authors: Gibson, Joel; Fieldhouse, Rachel; Chan, Melanie; Sadeghi-Alavijeh, Omid; Burnett, Leslie; Izzi, Valerio; Persikov, Anton; Gale, Daniel; Storey, Helen ; Savige, Judy

Title: Prevalence Estimates of Predicted Pathogenic COL4A3 â€" COL4A5 Variants in a Population Sequencing Database and Their Implications for Alport Syndrome

Running title: Prevalence of COL4A3-COL4A5 variants

Manuscript Type: Original Article - Basic Research

Manuscript Category: Genetics and development

Funders: FUNDREF :No data available.

Financial Disclosure: No D. Gale reports Honoraria from Alexion, Otsuka; and Other Interests/Relationships as a Trustee for AlportUK. J. Savige reports Other Interests/Relationships with Alport Foundation Australia, and the PKD Australia Scientific Board. L. Burnett reports Research Funding from Takeda (Shire): Investigator Initiated Grant for genomic screening of Gaucher disease Scientific Advisor or Membership as a Member of Genetics Advisory Committee, Royal College of Pathologists of Australasia (RCPA), Member of Genetics Services Executive Committee, NSW Health Genetics Network, Member of Community Genetics NSW Advisory Committee, These roles are honorary; Other Interests/Relationships as a Member of KidGen Renal Genetics Flagship of Australian Genomics, Member of CIRCA Clinical Immunogenomics Research Consortium Australasia, Non-Executive Director, AACB Services Pty Ltd, Australasian Association of Clinical Biochemists, These roles are honorary. The remaining authors have no disclosures.

Study Group/Organization Name: CUST_STUDY_GROUP/ORGANIZATION_NAME :No data available.

Study Group Members' Names: CUST_STUDY_GROUP_MEMBERS :No data available.

\section{Total number of words: 3800}

Abstract: $<b>$ Background: $</ b>$ The prevalence of Alport syndrome varies from one in 5,000 to one in 53,000 . This study estimated the frequencies of predicted pathogenic <i>COL4A3â $€^{\prime \prime} \operatorname{COL} 4 A 5</ i>$ variants in sequencing databases of populations without known kidney disease.

$\langle b>$ Methods $:</ b>$ Predicted pathogenic variants were identified using filtering steps based on the ACMG/AMP criteria that considered collagen IV Î $\pm 3 a ̂ €^{\prime \prime} \mid \hat{ \pm} \pm 5$ position 1 Gly to be critical domains. The population frequencies of predicted pathogenic $<i>C O L 4 A 3 a ̂ \epsilon^{\prime \prime} C O L 4 A 5</ i>$ variants were then determined per mean number of sequenced alleles. Population frequencies for compound heterozygous and digenic combinations were calculated from the results for heterozygous variants.

$<\mathrm{b}>$ Results: $</ \mathrm{b}><\mathrm{i}>\mathrm{COL} 4 \mathrm{~A} 3 \mathrm{a} \epsilon^{\prime \prime} \mathrm{COL} 4 \mathrm{~A} 5</ \mathrm{i}>$ variants resulting in position $1 \mathrm{Gly}$ substitutions were confirmed associated with haematuria ( $p$ each $<0.0001$ ). Predicted pathogenic COL4A5 variants were found in at least one in 2,320 individuals. p.(Gly624Asp), represented nearly half $(16 / 33,48 \%)$ the variants in Europeans. Most $<i>\operatorname{COL} 4 A 5</ i>$ variants $(54 / 59,92 \%)$ had a biochemical feature that potentially mitigated clinical impact. 
Predicted pathogenic heterozygous $<\mathrm{i}>\mathrm{COL} 4 \mathrm{~A} 3</ \mathrm{i}>$ and $<\mathrm{i}>\mathrm{COL} 4 \mathrm{~A} 4</ \mathrm{i}>$ variants affected one in 106 of the population, consistent with the finding of Thin basement membrane nephropathy in normal donor kidney biopsies. Predicted pathogenic compound heterozygous variants occurred in one in 88,866 individuals and digenic variants in at least one in 44,793.

$<b>$ Conclusions: $</ b>$ The population frequencies for Alport syndrome are suggested by the frequencies of predicted pathogenic $<i>\operatorname{COL} 4 A 3 a ̂ €^{\prime \prime} C O L 4 A 5</ i>$ variants but must be adjusted for the disease penetrance of individual variants, as well as the likelihood of already diagnosed disease and non-Gly substitutions. Disease penetrance may depend on other genetic and environmental factors. 


\section{Significance Statement}

The population frequencies of Alport syndrome vary greatly in different reports. This study examined a population sequencing database of individuals not known to have kidney disease using filtering steps corresponding to the ACMG/AMP criteria for 'predicted pathogenic' variants in COL4A3-COL4A5 that considered collagen chain position 1 Gly residues 'critical domains.' Predicted pathogenic COL4A5 variants occurred in at least one in 2,320 individuals. Heterozygous COL4A3 or COL4A4 variants affected one in 106; compound heterozygous COL4A3 or COL4A4 variants affected one in 88,866 . The actual prevalences are even greater since they also include already-diagnosed disease and other variants not examined here. The high frequency of predicted pathogenic COL4A3- COL4A5 variants suggests that other genetic and environmental factors mitigate the corresponding clinical manifestations of disease. 


\title{
Prevalence Estimates of Predicted Pathogenic COL4A3 - COL4A5 Variants in a Population Sequencing Database and their Implications for Alport Syndrome
}

\author{
${ }^{1}$ Joel Gibson, ${ }^{2}$ Rachel Fieldhouse, ${ }^{3,4}$ Melanie MY Chan, ${ }^{3,4}$ Omid Sadeghi-Alavijeh, \\ ${ }^{2}$ Leslie Burnett, ${ }^{5}$ Valerio Izzi, ${ }^{6}$ Anton V Persikov, ${ }^{4}$ Genomics England Research \\ Consortium, ${ }^{3,4}$ Daniel P Gale, ${ }^{7}$ Helen Storey, ${ }^{1}$ Judy Savige \\ ${ }^{1}$ The University of Melbourne Department of Medicine, Melbourne Health and \\ Northern Health, Royal Melbourne Hospital, Parkville VIC 3050 AUSTRALIA; \\ ${ }^{2}$ Kinghorn Centre for Clinical Genomics, Garvan Institute of Medical Research, \\ Darlinghurst, NSW, 2010, AUSTRALIA; ${ }^{3}$ Department of Renal Medicine, University \\ College London, London, UK; ${ }^{4}$ Genomics England, Queen Mary University of \\ London, London, UK; ${ }^{5}$ Center for Cell-Matrix Research and Biocenter Oulu, \\ University of Oulu, FINALND;' 6 Lewis-Sigler Institute for Integrative Genomics, \\ Princeton University, New Jersey, 08544, USA; ${ }^{7}$ Molecular Genetics, Viapath \\ Laboratories, $5^{\text {th }}$ floor Tower Wing, Guy's Hospital, London, SE1 9RT, UK.
}

Short running title: Population Frequency of Alport Syndrome

\author{
Address for Correspondence: \\ Prof Judy Savige \\ The University of Melbourne \\ Department of Medicine (Melbourne Health and Northern Health) \\ Royal Melbourne Hospital \\ Parkville VIC 3050 \\ AUSTRALIA \\ Email: jasavige@unimelb.edu.au \\ Tel: + 61383443260
}




\begin{abstract}
Background: The prevalence of Alport syndrome varies from one in 5,000 to one in 53,000 . This study estimated the frequencies of predicted pathogenic COL4A3COL4A5 variants in sequencing databases of populations without known kidney disease.

Methods: Predicted pathogenic variants were identified using filtering steps based on the ACMG/AMP criteria that considered collagen IV $\square 3-\square 5$ position 1 Gly to be critical domains. The population frequencies of predicted pathogenic COL4A3COL4A5 variants were then determined per mean number of sequenced alleles. Population frequencies for compound heterozygous and digenic combinations were calculated from the results for heterozygous variants.
\end{abstract}

Results: COL4A3-COL4A5 variants resulting in position 1 Gly substitutions were confirmed associated with haematuria ( $p$ each $<0.0001$ ). Predicted pathogenic COL4A5 variants were found in at least one in 2,320 individuals. p.(Gly624Asp), represented nearly half $(16 / 33,48 \%)$ the variants in Europeans. Most COL4A5 variants $(54 / 59,92 \%)$ had a biochemical feature that potentially mitigated clinical impact.

Predicted pathogenic heterozygous COL4A3 and COL4A4 variants affected one in 106 of the population, consistent with the finding of thin basement membrane nephropathy in normal donor kidney biopsies. Predicted pathogenic compound heterozygous variants occurred in one in 88,866 individuals and digenic variants in at least one in 44,793 .

Conclusions: The population frequencies for Alport syndrome are suggested by the frequencies of predicted pathogenic COL4A3-COL4A5 variants, but must be adjusted for the disease penetrance of individual variants as well as for the likelihood of already diagnosed disease and non-Gly substitutions. Disease penetrance may depend on other genetic and environmental factors. 


\section{Introduction}

Alport syndrome is an inherited renal disease characterised by progressive kidney failure, hearing loss, lenticonus and fleck retinopathy ${ }^{1}$. X-linked $(X L)$ inheritance results from pathogenic variants in $C O L 4 A 5^{2}$, and autosomal recessive (AR) disease from two pathogenic variants in COL4A3 or COL4A4 in trans ${ }^{3}$. The COL4A3 - COL4A5 genes code for the collagen IV $\square \square, \square \square$ and $\square \square$ chains that trimerise to form a network that represents the major component of the basement membranes of the glomerulus, cochlea, lens and retina.

Individuals with heterozygous pathogenic COL4A3 or COL4A4 variants are carriers for AR Alport syndrome and sometimes diagnosed with Thin basement membrane nephropathy 4,5 , even without a renal biopsy being performed. The term 'autosomal dominant' (AD) Alport syndrome is also used but affected individuals typically have persistent haematuria and not the hearing loss, ocular abnormalities, or typical glomerular basement membrane (GBM) lamellation ${ }^{6}$. In contrast to XL and AR disease, the risk of renal failure for heterozygous pathogenic COL4A3 or COL4A4 variants is uncertain because most reported series comprise only hospital-based patients ${ }^{7}$. Digenic Alport syndrome occurs with heterozygous pathogenic variants in two different $C O L 4 A 3-C O L 4 A 5$ genes $^{8}$, and clinical features are more severe than with only a heterozygous $C O L 4 A 3$ or $C O L 4 A 4$ variant ${ }^{8}$. In addition, any variant or combination of variants in COL4A3 - COL4A5 may result in focal and segmental glomerulosclerosis probably secondary to a defective GBM and podocyte $\operatorname{loss}^{9,10}$.

Alport syndrome has been considered rare because it affects fewer than one in 2,000 individuals $^{11}$, and the US has less than 200,000 cases $^{7}$. Its exact prevalence is not known ${ }^{12}$. Population-based studies have suggested frequencies of one in 5,000 in Utah ${ }^{13}$; one in 17,000 in southern Sweden ${ }^{14}$; and one in 53,000 in Finland ${ }^{15}$. However persistent haematuria and GBM thinning that correspond to heterozygous COL4A3 or COL4A4 variants occur in $1-2.5 \%$ of children and adults on population screening ${ }^{16,17}$, and, depending on the definition of thinning, in $4 \%$ to $7 \%$ of normal donor kidney transplant biopsies ${ }^{18}$ respectively.

Alport syndrome is suspected where persistent haematuria occurs together with a family history of haematuria or renal impairment; or an abnormal GBM appearance or collagen IV chain composition ${ }^{19}$. Haematuria is highly penetrant ${ }^{20,21}$ but many affected individuals are undiagnosed. These are often men with X-linked disease and late onset kidney failure, or where there is no family history or the kidney biopsy is too scarred to demonstrate the characteristic features. Although women with $X-$ linked disease are affected twice as often as men their recognition is often even more problematic ${ }^{22}$, because they often have only haematuria and a thinned rather than lamellated $\mathrm{GBM}^{22}$.

The most sensitive method for the diagnosis of Alport syndrome is genetic testing ${ }^{19}$, ${ }^{23}$ with Massively Parallel Sequencing, which detects causative variants in up to $90 \%$ of cases $^{23}$. Identifying likely pathogenic variants in the COL4A3-COL4A5 genes in a dataset of individuals not known to have kidney disease, such as gnomAD, gnomAD controls, Exome Variant Server (EVS) or TOPMed, indicates more accurately the population frequencies of undiagnosed COL4A5, heterozygous COL4A3 or COL4A4 variants, and hence compound heterozygous and digenic disease. However these databases share some datasets and include no clinical information. Conversely, the 
Genomics England 100,000 Genomes Project is an independent dataset that includes clinical information but its recruitment of individuals with inherited kidney disease precludes its use in determining population frequencies.

Genomic variants are usually assessed for pathogenicity using the American College of Medical Genetics and Genomics/Association for Molecular Pathology (ACMG/AMP) criteria ${ }^{24}$. Nonsense, canonical splice site variants and frameshift changes in the COL4A3 - COL4A5 genes are likely to be pathogenic since loss of function is a recognised disease mechanism for these genes ${ }^{24}$. However the commonest variants are missense (databases.lovd.nl/ shared/genes/). The interpretation of missense variants from a population not known to have kidney disease is complicated by the absence of clinical, segregation, and functional data (Suppl Table 1), and compounded by the lack of expert panel assertions for most variants (https://clinvarminer.genetics.utah.edu/).

However affecting a 'critical domain' also represents one of the ACMG/AMP criteria for pathogenicity (PM1) ${ }^{24}$, and in osteogenesis imperfecta, position 1 Gly residues in the collagen I $\square 1$ chain Gly-Xaa-Yaa repeats are considered critical because of their disruptive effect on the molecule ${ }^{25}$. Gly is the smallest amino acid, position 1 Gly residues are found within the interior of the collagen triple helix, and their replacement by any larger residues disturbs the helix formation. The commonest reported missense variants in COL4A3 - COL4A5 result in position $1 \mathrm{Gly}$ substitutions in the intermediate domain of the collagen IV $\square 3$, $\square 4$ and $\square 5$ chains ${ }^{12}$, ${ }^{25}$, and the recent Chandos House meeting of the Alport Variant Consortium recommended that these are generally considered pathogenic ${ }^{26}$.

There are nevertheless also important differences in the biochemistry of collagen IV and I. The collagen IV chains retain their non-collagenous amino and carboxy termini, and include multiple non-collagenous interruptions, in the Gly-Xaa-Yaa repeats of the intermediate domains (Suppl Table 2) ${ }^{27-29}$. Gly substitutions in the amino and carboxy termini, the interruptions and positions 2 and 3 in the Gly-XaaYaa- repeats ${ }^{29}$ are not considered critical ${ }^{25,30 .}$

This study used the UK 100,000 Genomes Project to confirm that predicted pathogenic position 1 Gly substitutions in the collagen IV $\square 3$, $\square 4$ and $\square 5$ chains were associated with haematuria. It then identified predicted pathogenic COL4A5 variants in the large, unbiased gnomAD2.1.1 dataset and confirmed the strategy using the gnomad control, EVS and TOPMed cohorts. The amount of overlap between the datasets was determined from the number of shared variants. A similar approach has been used previously to determine the population frequency of polycystic kidney and liver disease ${ }^{31}$.

This study then identified predicted pathogenic COL4A3 and COL4A4 variants in gnomAD, and used these to calculate the frequencies of compound heterozygous and digenic variants. These population frequencies provide an approximate guide to the prevalence of $\mathrm{XL}, \mathrm{AR}$ (compound heterozygous) and digenic Alport syndrome and Thin basement membrane nephropathy but the precise prevalence must also take into account the penetrance of individual variants as well as the occurrence of already diagnosed disease and missense variants due to non-Gly substitutions. 


\section{Methods \\ Datasets \\ gnomAD. The Genome Aggregation Database (gnomADv2.1.1,} www.gnomAD. broadinstitute.org) ${ }^{32}$ is a large dataset that includes COL4A3 - 5 alleles from unrelated adults with equal numbers of males and females who have undergone exomic (in about $90 \%$ ) or genomic sequencing in disease and population studies. It includes individuals with adult-onset diseases such as diabetes, cardiac disease and psychiatric conditions, and excludes those with severe paediatric diseases and their close family members. Participants were not known to have inherited kidney disease and individuals with any severe disease were expected less frequently than in the general population. gnomAD indicates variants that are homozygous but variants are not otherwise linked to an individual and it is not possible to identify a different second variant in COL4A3 - COL4A5 and hence compound heterozygous or digenic variants directly. Ancestries are available but the clinical phenotypes are not recorded.

Three other datasets were also examined for predicted pathogenic variants in COL4A5. These were the gnomAD controls (vs2.1.1, www.gnomAD.broadinstitute.org) which comprises alleles from the normal individuals, recruited as controls for gnomAD studies; Exome Variant Server (EVS) (www.evs.gs.washington.edu/EVS/) which includes alleles from exomic sequencing of individuals recruited for mainly cardiac and pulmonary studies; and TOPMed (www.bravo.sph.umich.edu/freeze8/hg38/) with alleles from genomic sequencing of individuals recruited for heart, lung, blood and sleep studies. Although participants in these cohorts were not known to have inherited kidney disease, they had not been specifically phenotyped for renal disease and it was uncertain how many had underlying renal disease or impaired kidney function. The gnomAD dataset includes all the 'gnomAD control' cohort, and some of the studies in the other cohorts. The amount of overlap was assumed to include the COL4A3 - COL4A5 variants randomly and was quantitated from the number of shared variants with the same gender.

In contrast, the Genomics England 100,000 Genomes Project database (www.genomicsengland.co.uk/) ${ }^{33}$ includes whole genome sequencing data from individuals with rare diseases including inherited kidney disease and their unaffected relatives. This dataset also includes clinical phenotypes including haematuria noted in the hospital records. This database was used to examine whether substitutions affecting position 1 Gly substitutions in the collagen IV $\square 3, \square 4$ and $\square 5$ chains were associated with haematuria.

Ethics approval was obtained at the time of recruitment into participation in each of the constituent studies. The databases are freely-accessible online and were examined between 24 December 2019 and 1 February 2021.

\section{COL4A3-5 variants}

COL4A5 variants were described using the GRCh37/hg19 sequence with 53 exons and the reference sequence for the collagen IV $\square 5$ chain (NM_033380.2, LRG_232t2). COL4A3 and COL4A4 variants were described using the GRCh37/hg19 sequence and the reference sequences for collagen IV $\alpha 3$ and $\alpha 4$ chains (NM 000091.4, LRG_230t1) and (NM 000092.4, LRG_231t1,) respectively. 
The locations of the non-collagenous termini and interruptions in the collagenous domains of each chain were noted (Suppl Table 2) ${ }^{27-29}$.

\section{Filtering steps to determine predicted pathogenic variants based on the ACMG/AMP criteria}

Variants were filtered to exclude variants that failed any step in order to be confident of the corresponding population frequencies. Variants were filtered according to their effect on the canonical transcripts using the following steps and ACMG criteria

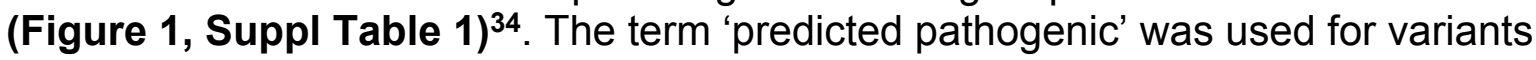
that were not excluded, to differentiate them from the 'pathogenic' and 'likely pathogenic' classes derived from the ACMG/AMP classification in individuals with clinical features of disease.

- Synonymous variants and intronic variants other than those affecting a $+/-1$ or 2 splice site were excluded.

- Variants that resulted in a protein-truncating change, frameshift, or canonical splice site change were graded PVS1 because loss of function is a known disease mechanism for COL4A3 - COL4A5-associated disease ${ }^{24}$.

- Missense variants resulting in position 1 Gly substitutions within the intermediate collagenous domains of the collagen IV $\square \square \square \square \square \square$ chains since missense variants are a common mechanism for COL4A3 - COL4A5-associated disease with a low rate of benign missense variation (PP2). Variants that resulted in a position 1 Gly substitution were considered to affect a critical and well-established functional domain (PM1).

- Missense variants were examined with individual computational tools: Polyphen2 ( PP2, score >0.8, www.genetics.bwh.harvard.edu/pph2/); SIFT (Sorting Intolerant from Tolerant, Deleterious or Polymorphism, www.sift.bii.a-star.edu.sg/); and Mutation Taster, (MT, Disease-causing, or Polymorphism, www.mutationtaster.org/). Missense variants were also examined for conservation among vertebrates (www.asia.ensembl.org/) (PP3).

- Variants occurred fewer than an arbitrary cut off of 30 times in gnomAD (PM2) ${ }^{34}$. The formula used to determine the cutoff is normally based on the disease prevalence and contribution of the gene which were not known ${ }^{35}$. While the cut-off varies for different genes, this level has been used for other heterogeneous genes resulting in $A D$ kidney disease.

- Variants were assessed for previous reports as Likely Pathogenic or Pathogenic in a reputable source such as ClinVar (www.ncbi.nlm.nih.gov/clinvar/) or LOVD (www.databases.lovd.nl/shared/genes/COL4A3-5 (PP5) despite this practice being controversial $^{36,37}$. Variants in ClinVar reported as Benign, Likely Benign, Variant of Uncertain Significance or Conflicting, were excluded. Variants in LOVD reported as Benign or Likely Benign were excluded. Variants that resulted in the same amino acid change as a previously-established pathogenic variant regardless of nucleotide change in ClinVar (www.ncbi.nlm.nih.gov/clinvar/) or LOVD

(www.databases.lovd.nl/shared/genes/COL4A3-5 were also noted (PS1). In these cases the submitters' evaluations were accepted since their assessments included the associated clinical data, that we were not able to access. Anyway all variants were assessed independently for pathogenicity using an objective online tool (Varsome). 
- Variants were examined to determine if they resulted in a novel missense change at an amino acid where a different change was previously reported as pathogenic (PM5).

All COL4A3-COL4A5 variants were also assessed by Varsome (www.varsome.com/) using the ACMG/AMP criteria ${ }^{38}$, and COL4A5 variants in gnomAD were assessed in Alamut (www.interactive-biosoftware.com/alamut-visual/). These semi-automated online tools were chosen because they are objective, not subject to individual laboratory practice, and are widely available. They used slightly different criteria from our filtering steps, for example, numerous computational tools, GERP scores for conservation, and weighting of scores. Variants assessed as Benign, Likely Benign or a Variant of Uncertain Significance (VUS) by Varsome or Alamut were excluded.

Predicted pathogenic COL4A3 - COL4A5 variants resulting in position 1 Gly substitutions in the 100,000 Genomes Project database and haematuria Individuals in the 100,000 Genomes Project database with haematuria were identified from haematuria-related Human Phenotype Ontology terms after excluding those with cancer of the kidney or bladder, or kidney stones. Predicted pathogenic variants resulting in a position 1 Gly substitution in COL4A3 - COL4A5 were identified from all Gly substitutions in these genes using our filtering strategy, and the total number of individuals with a predicted pathogenic variant were compared in the cohorts with haematuria and without (chi square with Yates' correction, two tailed, Graphpad).

Prevalence of predicted pathogenic variants in COL4A5, COL4A3 and COL4A4 The population frequencies of predicted pathogenic variants were calculated based on the mean number of alleles examined. For COL4A5, which is located on the $\mathrm{X}$ chromosome, the corresponding number of individuals was determined from the number of alleles after correcting for the number of males since they have only one allele for COL4A5. The total number of individuals recruited in the whole cohort was greater than the number calculated from the number of alleles examined because some regions of a gene were not sequenced or failed quality testing.

Prevalence of variants in different ancestries and founder variants

The frequencies of predicted pathogenic COL4A5, COL4A3 and COL4A4 variants were noted in people of different ancestries (African, Latino, Ashkenazi Jew, East Asian, European Finnish, European non-Finnish, South Asians and Others, who did not unambiguously cluster with any of the major populations by Principal Component Analysis). Any founder variants (arbitrarily set at $\geq 18$ alleles) were noted.

\section{Biochemical features likely to mitigate clinical phenotypes for predicted pathogenic COL4A5 variants}

The cohort with predicted pathogenic COL4A5 variants were then assessed for any biochemical features associated with clinically milder phenotypes that might explain the lack of previous diagnosis. Mitigating features included female gender; 22 location within the 20 amino terminal exons including the amino non-collagenous domain (204 residues) ${ }^{39}$; position adjacent to a non-collagenous domain or interruption 30 ; and Gly substitutions with a less disruptive residue, such as Ala, Ser or Cys 40, 41. 


\section{Calculation of population frequencies for heterozygous, compound heterozygous and digenic COL4A3 and COL4A4 variants}

The population frequencies of predicted pathogenic heterozygous COL4A3 and COL4A4 variants were derived from the sum of the allele frequencies for each variant assuming that they were inherited independently and that no or very few individuals had inherited two.

The population frequency of AR Alport syndrome represents the sum of the frequencies of predicted pathogenic homozygous and compound heterozygous variants in trans in COL4A3 plus COL4A4. Previous reports suggest homozygous inheritance represents $30 \%$ of all AR Alport syndrome ${ }^{42}$. Homozygous variants are indicated in gnomAD and usually result from relationships that are consanguineous or consanguineous by descent. However in Alport syndrome homozygous predicted pathogenic variants result in early onset kidney failure which would have precluded recruitment into gnomAD. The likelihood of individual homozygous $C O L 4 A 3$ or $C O L 4 A 4$ variants occurring by chance was very small and not calculated here.

It was not possible to identify compound heterozygous and digenic variants from the databases because variants were not linked to any individual. In addition compound heterozygous and digenic variants were likely to be underrepresented because they too are associated with early onset kidney failure. Thus the population frequencies of predicted pathogenic compound heterozygous variants in COL4A3 or COL4A4 were calculated simply from the likelihood of the occurrence of each allele occurring independently.

Digenic variants were calculated from two predicted pathogenic variants in COL $4 A 3$ and COLAA4 in cis or trans and from a predicted pathogenic variant in COLAA5 and either COL4A3 or COL4A4. 


\section{Results \\ Predicted pathogenic COL4A3- COL4A5 variants resulting in position 1 Gly substitutions in the 100,000 Genomes Project database and haematuria}

This cohort comprised 2,221 individuals with documented haematuria and 37,200 with none. It included 19 predicted pathogenic COL4A5 variants that affected a position 1 Gly and were associated with haematuria and 9 that were not, consistent with an association with haematuria (chi-square 95.83, $\mathrm{p}<0.0001$ ). COL4A3 and COL4A4 variants resulting in predicted pathogenic position 1 Gly substitutions were also associated with haematuria ( $p<0.0001$ for each, Table 1, Suppl Tables 3 - 5).

\section{COL4A5}

\section{Predicted pathogenic COL4A5 variants in gnomAD}

Fifty-four variants in COL4A5 (frameshift, nonsense and canonical splice site variants, position 1 Gly substitutions) were identified for further assessment (Table 2). These included one deletion resulting in a termination codon and 6 canonical splice site variants, all predicted pathogenic and found in 8 individuals. There were also 47 position 1 Gly substitutions, but 13 were excluded on filtering (including p.(Gly953Val), Suppl Table 3), resulting in 34 Gly substitutions in 51 individuals (Table 2).

Thus, gnomAD included 59 individuals with a predicted pathogenic COL4A5 variant in a mean allele number of 170,190 (113,460 individuals) or one in 1,923 individuals. If the population frequency were calculated from the total gnomAD cohort of 136,920 individuals at the time of examination, then the population frequency was one in 2,320 .

Two of the predicted pathogenic variants found in gnomAD (p.(Gly624Asp and p.(Gly752Val)) were also present in the 100,000 Genomes Project database but none of the 4 individuals with these variants had haematuria.

\section{Population frequencies of predicted pathogenic COL4A5 variants in different ancestries in gnomAD}

The population frequencies of predicted pathogenic COL4A5 variants differed in people of different ancestries (Table 3). Predicted pathogenic COL4A5 variants were more common in people of European (one in 1,800), African (1 in 1,733) or East Asian (1 in 2,310) ancestry. In general, COL4A5 variants were less common in Ashkenazim (1 in 4,961) and not found in Finns.

The commonest predicted pathogenic variant, p.(Gly624Asp), was considered Pathogenic by ClinVar**and has been reported pathogenic many times ${ }^{43}$. It was present 16 times in 59,512 individuals of European ancestry corresponding to a prevalence of one in 2,479 . Nearly half $(16 / 33)$ of all Europeans with a predicted pathogenic COL4A5 variant had p.(Gly624Asp) and it was not present in other ancestries. This variant is associated with late onset kidney failure in men ${ }^{44,45}$, and a $50 \%$ cumulative probability of end-stage kidney failure by the age of 54 , which is nearly 30 years later than with other variants ${ }^{46}$. The variant is located immediately adjacent to an interruption in the collagenous sequence and was found in 12 females and only 4 males, which are both factors that helped explain its association with a milder phenotype and why it had not been detected previously in individuals recruited into gnomAD. 


\section{Founder COL4A5 variants in gnomAD}

Most COL4A5 variants resulting in a Gly substitution were found once only but five were found more often, usually in a single ancestral group: p.(Gly624Asp) $(n=16)$ and p.(Gly626Ser)(n=7) in Europeans; p.(Gly961Val)(n=5) and p.(Gly1074Ser)(n=7) in Latinos; and p.(Gly953Val) in South and East Asians. All these variants are located adjacent to a non-collagenous interruption, and are less likely to be disruptive than other position 1 Gly substitutions (Suppl Table 2).

\section{Predicted pathogenic COL4A5 variants in other cohorts}

In the normal control subset of gnomAD comprising a mean of 87,876 alleles or 58,584 individuals, 24 individuals had 17 predicted pathogenic variants, occurring in one in 2,441 individuals (Table 2). All these variants were also found in the whole gnomAD cohort as expected.

In the EVS dataset comprising a mean of 10,563 alleles or 7,042 individuals, 3 individuals had predicted pathogenic variants, corresponding to a population frequency of one in 2,347 (Suppl Table 6). Five of the 8 COL4A5 variants found in the EVS dataset were also present with a consistent gender in gnomAD suggesting at most an overlap of $5 / 8(63 \%)$ variants.

In the TOPMed cohort of a mean of 125,568 alleles or 83,713 individuals, 40 individuals had predicted pathogenic variants, corresponding to a population frequency of one in 2,093 (Suppl Table 7). Only two of the 45 COL4A5 variants found in the TOPMed dataset (p.(Gly524Asp) and p.(Gly1424Ser)) were also present with a consistent gender in gnomAD suggesting very little if any overlap in the datasets. The population frequency of predicted pathogenic variants COL4A5 variants from TOPMed might therefore be considered independent and confirmatory of the gnomAD results.

Thus, the population frequencies of predicted pathogenic COL4A5 variants varied from one in 2,320 (based on total participants recruited) or one in 1,923 (based on alleles examined) in gnomAD, one in 2,441 in the gnomAD normal controls, one in 2,347 in EVS, and one in 2,093 in TOPMed.

\section{Mitigating features for phenotypes of predicted pathogenic COL4A5 variants in gnomAD}

Features that might mitigate the clinical features, for example, result in late onset kidney failure were found for 54 of the 59 COL $4 A 5$ variants (92\%). These included being identified in a woman $(41 / 59,69 \%)$; and for Gly substitutions: being located in the 20 amino exons $(11 / 51,22 \%)$ or adjacent to a non-collagenous interruption $(23 / 51,45 \%)$; or a Gly substitution with a less disruptive residue $(19,37 \%)$ (Suppl Table 8).

\section{COL4A3}

\section{Predicted pathogenic COL4A3 variants in gnomAD}

Five hundred and seventy-four variants $(2.6 \%)$ were identified in COL4A3 in gnomAD for further assessment. Fifteen position 1 Gly substitutions were excluded because evidence was not consistent with pathogenicity (Suppl Table 9). Overall there were 559 predicted pathogenic variants in a mean of $245,889(0.23 \%)$ alleles 
corresponding to a population frequency of $0.45 \%$. These variants were position 1 Gly substitutions ( $n=380$, in $68 \%$ of variants, $0.16 \%$ of the population), frameshift $(n=110,20 \%$ of the variants, $0.04 \%)$, nonsense $(n=33,6 \%$ of the variants, $0.01 \%)$ or splicing variants $(n=36,6 \%$ of the variants, $0.01 \%)$. No predicted pathogenic COL4A3 variant was found in the homozygous form.

In the total cohort of 136,930 individuals, the population frequency of a predicted pathogenic COL $4 A 3$ variant was $0.41 \%$.

\section{Population frequencies of predicted pathogenic COL4A3 variants in different ancestries}

Most predicted pathogenic COL4A3 variants were present in a single ancestry. Predicted pathogenic heterozygous COL4A3 variants were commonest in people of Latino $(0.80 \%)$ or East Asian (0.64\%) ancestries, and least common in Askenazim $(0.14 \%)$ and Finns $(0.14 \%)$ (Table 4).

\section{COL4A4}

\section{Predicted pathogenic COL4A4 variants in gnomAD}

Five hundred and ninety-nine variants were identified in COL4A4 in gnomAD for further assessment. Twenty-two position 1 Gly substitutions were excluded because evidence was not consistent with pathogenicity (Suppl Table 9). The variants p.(Gly545Ala) and p.(Gly999Glu) were considered Benign and are discussed in the footnote to Suppl Table 9.

In COL4A4, there were 577 predicted pathogenic variants in in a mean of 233,916 alleles $(0.25 \%$ alleles $)$ corresponding to a population frequency of $0.49 \%$. Variants were position 1 Gly substitutions ( $n=458,82 \%$ of all variants, in $0.20 \%$ of the population), frameshift $(n=42,0.02 \%)$, nonsense $(n=48,0.02 \%)$ or splicing variants $(n=29,0.01 \%)$. No homozygous predicted pathogenic COL4A4 variant was found.

In the total cohort of 136,930 individuals, the population frequency of a predicted pathogenic COL $4 A 4$ variant was $0.42 \%$.

\section{Population frequencies of predicted pathogenic COL4A4 variants in different ancestries}

Heterozygous predicted pathogenic COL4A4 variants were commonest in people of Latino $(0.78 \%)$ and East Asian $(0.70 \%)$ ancestries, and least common in Finns $(0.10 \%)$ and Ashkenazim (0.18\%) (Table 4).

Most founder variants were found once only in any ancestral group, but there were also 7 more common COL4A4 founder variants, which were all position 1 Gly substitutions (Suppl Table 10). These were mainly found in one population each including Africans (p.(Gly445Ala), Latinos (p.(Gly481Ser), East Asians (p.(Gly816Glu), non-Finnish Europeans (p.(Gly1178Ser), p.(Ser969Ter) and Others (p.(Gly774Arg)). No founder variants were found in Ashkenazim or Finns. p.(Ser969Ter) was common in Europeans, occurring in 18 alleles of the 280,956 examined or one in 15,608 individuals but otherwise no founder variants were frameshift or splicing variants. 


\section{Population frequencies of heterozygous predicted pathogenic COL4A3 and COL4A4 variants}

The population frequency of heterozygous predicted pathogenic variants in COL4A3 or COL4A4 was $0.94 \%$ for all individuals (Suppl Table 11).

Heterozygous predicted pathogenic COL4A3 and COL4A4 variants were commonest in people of Latino (in 1.58\%), East Asian (1.34\%) or African (1.28\%) ancestries, and least common in Finns (0.24\%) and Ashkenazim (0.32\%).

\section{Association of heterozygous predicted pathogenic COL4A3 and COL4A4 variants in gnomAD with haematuria in the 100,000 Genomes Project dataset} Seventeen COL4A3 and 27 COL4A4 predicted pathogenic Gly substitutions found in gnomAD were also present in the 100,000 Genomes database. Fourteen individuals of the 2,221 with haematuria had one of these variants and 78 of the 37,200 without haematuria also had one (chi squared 14.17, $p=0.0002$ ) which indicated that these variants were associated with haematuria. This confirmed that the heterozygous predicted pathogenic COL4A3 and COL4A4 variants resulting in Gly substitutions found in in gnomAD were associated with haematuria.

\section{Calculated population frequencies of compound heterozygous and digenic COL4A3 and COL4A4 variants}

The population frequency of $0.94 \%$ for heterozygous predicted pathogenic COL $4 A 3$ or COL4A4 variants corresponded to about $0.9 \%$ of the population or one in 106 people.

The population frequency of two compound heterozygous predicted pathogenic variants in COL4A3 or COL4A4 was $1.13 \times 10^{-5}$, or $0.001 \%$, or 1 in 88,866 when the variants occurred independently.

The population frequency of a digenic predicted pathogenic COL4A3 and a COL4A4 variant was $2.23 \times 10^{-5}$ or $0.002 \%$ or 1 in 44,793 if each variant were in cis or in trans and the variants occurred independently. Digenic predicted pathogenic variants in COL4A5 plus COL4A3 or COL4A4 were much less common occurring in about one in 2,320 (for COL4A5) $x$ one in 106 (for COL4A3 or COL4A4) or one in 245,920 of the population. 


\section{Discussion}

Previous estimates of the population frequency of X-linked Alport syndrome have differed ${ }^{13-15}$, but examination of a large variant database from people without known kidney disease predicted pathogenic COL4A5 variants in at least one in 2,320 individuals and heterozygous pathogenic COL4A3 or COL4A4 variants of one in 106. The accuracy of population frequencies for these variants is suggested by the consistency of predicted pathogenic COL4A5 variants in a control subset, an overlapping cohort, and an independent dataset. However the population frequencies of the corresponding diseases while directly related to the prevalence of the predicted pathogenic COL4A3 - COL4A5 variants, must be corrected for the penetrance of clinical features for individual variants. Penetrance probably depends on other genetic and other factors, and we demonstrated for most COL4A5 variants that there were mitigating features that explained why variants had not been detected previously.

The population frequency of one in 106 for heterozygous predicted pathogenic variants in COL4A3 and COL4A4 corresponded to a population frequency for compound heterozygous COL4A3 or COL4A4 variants of one in 88,866 , and of digenic variants in each of COL4A3 and COL4A4 of one in 44,793, and a lesser contribution from COL4A5 and COL4A3 or COL4A4. The calculated frequency for compound heterozygous variants was less than expected for AR Alport syndrome ${ }^{7}$ which previous reports have suggested affects one in 6 Alport families ${ }^{47}$, or one in 40,000 of the population ${ }^{7,48}$. Recessive Alport syndrome results from compound heterozygous variants that occur by chance and from homozygous variants due to consanguinity or consanguinity by descent which account for a further $30 \%$ of cases ${ }^{42}$. Thus the overall population frequency for AR Alport syndrome must also take into account a further $30 \%$ from homozygous variants. Digenic COL4A3 - COL4A5 variants occur together by chance and the calculated frequencies are more accurate.

These population frequencies of predicted pathogenic variants are still underestimates since they do not include individuals with already diagnosed disease, with the large deletions or deep intronic splicing variants that are not detected with Whole Exome Sequencing ${ }^{23}$, or with pathogenic non- Gly substitutions, that were not evaluated ${ }^{12,49}$.

So what are the implications of these results for the prevalence of different modes of inheritance of Alport syndrome? In general, pathogenic COL4A5 variants are highly penetrant for haematuria and renal failure in males ${ }^{20}$, and for haematuria in females ${ }^{21}$. This study only examined high risk predicted pathogenic variants, that is, nonsense variants and position 1 Gly substitutions, but even so, many had biochemical features that potentially mitigated the clinical phenotype.

Interestingly, the population frequency of heterozygous predicted pathogenic COL $4 A 3$ and COL4A4 variants approximated the $1 \%$ estimated from the prevalence of persistent haematuria $^{16,17}$ and Thin membrane nephropathy in normal donor kidney biopsies ${ }^{18}$. However the penetrance of persistent haematuria with COL4A3 and COL4A4 variants is about $70 \%{ }^{7}$, and the penetrance of a thinned GBM, FSGS and renal impairment are not known. This means that the population frequencies of Thin basement membrane nephropathy or AD Alport syndrome deduced from the prevalence of heterozygous predicted pathogenic COL4A3 or COL4A4 variants must be adjusted for the incomplete penetrance associated with these variants. The prevalence of autosomal recessive Alport syndrome due to compound heterozygous and of digenic Alport syndrome is probably affected less because haematuria and renal impairment are more likely with two COL4A3 - COL4A5 variants ${ }^{8,50}$. 
Genetic testing is the most sensitive method for the detection of COL4A3 - 5 variants and hence for detection of the different forms of Alport syndrome ${ }^{19}$. Examination of data from the UK 100,000 Genomes Project confirmed that heterozygous predicted pathogenic position 1 Gly substitutions in COL4A5, COL4A3 or COL4A4 were each associated with haematuria and that these Gly residues were critical domains. While some position 1 Gly substitutions in COL4A3 - COL4A5 were not considered pathogenic and assertions from collagen I variants in osteogenesis imperfecta suggest that nearby amino acids determine whether chain flexibility from a non-Gly substitution is tolerated ${ }^{51}$.

The gnomAD and TOPMed datasets represented cohorts of individuals not known to have kidney disease that were used to determine the population frequencies of predicted pathogenic COLAA3 - COL4A5 variants. Sequencing was not available for all study participants at each residue, and in general the population frequencies were calculated from the mean number of alleles examined and the deduced number of individuals. However we have cited the more conservative value of COL4A5 variants in the whole cohort. There were also limitations in applying the ACMG/AMP criteria ${ }^{24}$ to variants where clinical data were not available, but while our strategy may have excluded some variants that were pathogenic, these small numbers were unlikely to have significantly affected the overall frequencies.

The large number of undetected COL4A5 variants identified in gnomAD may be explained by mitigating features that the associated clinical features were mild, such as haematuria or late onset kidney failure without extrarenal features. Thus, predicted pathogenic variants were found twice as often in women, which was consistent with $\mathrm{XL}$ inheritance, but different from the female representation in most previous reports ${ }^{12-14}$. Affected women often have only haematuria, but identifying affected women is still important because of their own risk of endstage kidney failure, because half their sons develop kidney failure ${ }^{22}$, and because treatment is effective ${ }^{52}$. Most other COL4A5 variants identified in gnomAD were associated with a clinically-mitigating feature such as location in the amino terminus or adjacent to an interruption, or a Gly substitution with Ala, Ser or Cys.

This study also demonstrated that the population frequencies of predicted pathogenic COL4A3 - COL4A5 variants varied in people of different ancestries. Predicted pathogenic variants in COL4A5 were more common in people from a European or African background, present at a low level in Ashkenazim and absent from Finns. This was consistent with the previouslyreported low population frequency in Finns ${ }^{15}$. In addition, heterozygous predicted pathogenic COL4A3 and COL4A4 variants affected more than $1 \%$ of people of Latino, African and East Asian ancestries, which was at least four times more common than in Ashkenazim and Finns. It may not be that $C O L 4 A 3-C O L 4 A 5$ variants are more common in some ancestries but rather that they are uncommon in others due to geographic and cultural isolation ${ }^{53}$.

In conclusion, predicted pathogenic COL4A5 variants occur at least one in 2,320 of the population but are often unrecognised. Heterozygous predicted pathogenic $C O L 4 A 3$ or COL4A4 variants occur in at least one in 106 of the population. The true prevalence of potentially pathogenic $C O L 4 A 3-C O L 4 A 5$ variants is even greater since they must also include already-diagnosed disease, variants that were not detected with exomic sequencing as well as non-Gly substitutions. However the prevalence of the different forms of inheritance of Alport syndrome depends on the penetrance of haematuria and renal impairment with these variants. Future studies should address penetrance of these clinical features. 


\section{Author contributions}

JG undertook the initial analysis and made major contributions to the initial draft. RF, LB, VI, AP and HS devised the list of interruptions, provided associated disease phenotypes, and helped in the assessments of predicted pathogenicity. MMYC, OSA and DPG helped with the acquisition of data, analysis and interpretation of data, and with revisions. VI, AP and HS also contributed to the acquisition of data, analysis and interpretation of data. JS designed the study, undertook some of the analysis, and drafted and revised the manuscript. All authors contributed to the writing, have approved the final submission and agree to be accountable for the data. JG undertook this study while an undergraduate student at the University of Melbourne.

\section{Disclosures}

D. Gale reports Honoraria from Alexion, Otsuka; and Other Interests/Relationships as a Trustee for AlportUK. J. Savige reports Other Interests/Relationships with Alport Foundation Australia, and the PKD Australia Scientific Board. L. Burnett reports Research Funding from Takeda (Shire): Investigator Initiated Grant for genomic screening of Gaucher disease Scientific Advisor or Membership as a Member of Genetics Advisory Committee, Royal College of Pathologists of Australasia (RCPA), Member of Genetics Services Executive Committee, NSW Health Genetics Network, Member of Community Genetics NSW Advisory Committee, These roles are honorary; Other Interests/Relationships as a Member of KidGen Renal Genetics Flagship of Australian Genomics, Member of CIRCA Clinical Immunogenomics Research Consortium Australasia, Non-Executive Director, AACB Services Pty Ltd, Australasian Association of Clinical Biochemists, These roles are honorary. The remaining authors have no disclosures.

\section{Acknowledgements}

The authors would like to thank the Genome Aggregation Database (gnomAD) and the groups that provided exome and genome variant data to this resource. A full list of contributing groups can be found at http://gnomad.broadinstitute.org/about. The authors would like to thank the NHLBI GO Exome Sequencing Project and its ongoing studies that produced and provided exome variant calls for comparison: the Lung GO Sequencing Project (HL-102923), the WHI Sequencing Project (HL-102924), the Broad GO Sequencing Project (HL-102925), the Seattle GO Sequencing Project (HL-102926) and the Heart GO Sequencing Project (HL-103010). Finally, the authors would also like to thank the hosts of the many websites used to evaluate variants for predicted pathogenicity, Varsome in particular, and the LOVD database, its contributors and administrators.

This research was also made possible through access to the data and findings generated by the 100,000 Genomes Project. The 100,000 Genomes Project is managed by Genomics England Limited (a wholly owned company of the Department of Health and Social Care). The 100,000 Genomes Project is funded by the National Institute for Health Research and NHS England. The Wellcome Trust, Cancer Research UK and the Medical Research council have also funded research infrastructure. The 100,000 Genomes Project uses data provided by patients and collected by the National Health Service as part of their care and support. 


\section{References}

1. Gubler, M, Levy, M, Broyer, M, Naizot, C, Gonzales, G, Perrin, D, Habib, R: Alport's syndrome. A report of 58 cases and a review of the literature. Am J Med, 70: 493-505, 1981.

2. Barker, DF, Hostikka, SL, Zhou, J, Chow, LT, Oliphant, AR, Gerken, SC, Gregory, MC, Skolnick, MH, Atkin, $\mathrm{CL}$, Tryggvason, $\mathrm{K}$ : Identification of mutations in the COL4A5 collagen gene in Alport syndrome. Science, 248: 1224-1227, 1990.

3. Mochizuki, T, Lemmink, HH, Mariyama, M, Antignac, C, Gubler, MC, Pirson, Y, Verellen-Dumoulin, C, Chan, B, Schroder, $\mathrm{CH}$, Smeets, HJ, et al.: Identification of mutations in the alpha 3(IV) and alpha 4(IV) collagen genes in autosomal recessive Alport syndrome. Nat Genet, 8: 77-81, 1994.

4. Buzza, M, Wilson, D, Savige, J: Segregation of hematuria in thin basement membrane disease with haplotypes at the loci for Alport syndrome. Kidney Int, 59: 1670-1676, 2001.

5. Buzza, M, Dagher, H, Wang, YY, Wilson, D, Babon, JJ, Cotton, RG, Savige, J: Mutations in the COL4A4 gene in thin basement membrane disease. Kidney Int, 63: 447-453, 2003.

6. Savige, J: Should We Diagnose Autosomal Dominant Alport Syndrome When There Is a Pathogenic Heterozygous COL4A3 or COL4A4 Variant? Kidney Int Rep, 3: 1239-1241, 2018.

7. Savige, J, Rana, K, Tonna, S, Buzza, M, Dagher, H, Wang, YY: Thin basement membrane nephropathy. Kidney Int, 64: 1169-1178, 2003.

8. Mencarelli, MA, Heidet, L, Storey, H, van Geel, M, Knebelmann, B, Fallerini, C, Miglietti, N, Antonucci, MF, Cetta, F, Sayer, JA, van den Wijngaard, A, Yau, S, Mari, F, Bruttini, M, Ariani, F, Dahan, K, Smeets, B, Antignac, C, Flinter, F, Renieri, A: Evidence of digenic inheritance in Alport syndrome. J Med Genet, 52: 163-174, 2015.

9. Voskarides, K, Damianou, L, Neocleous, V, Zouvani, I, Christodoulidou, S, Hadjiconstantinou, V, Ioannou, K, Athanasiou, Y, Patsias, C, Alexopoulos, E, Pierides, A, Kyriacou, K, Deltas, C: COL4A3/COL4A4 mutations producing focal segmental glomerulosclerosis and renal failure in thin basement membrane nephropathy. J Am Soc Nephrol, 18: 3004-3016, 2007.

10. Wickman, L, Hodgin, JB, Wang, SQ, Afshinnia, F, Kershaw, D, Wiggins, RC: Podocyte Depletion in Thin GBM and Alport Syndrome. PLoS One, 11: e0155255, 2016.

11. Baldovino, S, Moliner, AM, Taruscio, D, Daina, E, Roccatello, D: Rare Diseases in Europe: from a Wide to a Local Perspective. Isr Med Assoc J, 18: 359-363, 2016.

12. Hertz, JM, Thomassen, M, Storey, H, Flinter, F: Clinical utility gene card for: Alport syndrome - update 2014. Eur J Hum Genet, 23, 2015.

13. Hasstedt, SJ, Atkin, CL: X-linked inheritance of Alport syndrome: family P revisited. Am J Hum Genet, 35 : 1241-1251, 1983.

14. Persson, U, Hertz, JM, Wieslander, J, Segelmark, M: Alport syndrome in southern Sweden. Clin Nephrol, 64: 85-90, 2005.

15. Pajari, $\mathrm{H}$, Kaariainen, $\mathrm{H}$, Muhonen, T, Koskimies, O: Alport's syndrome in 78 patients: epidemiological and clinical study. Acta Paediatr, 85: 1300-1306, 1996.

16. Dodge, WF, West, EF, Smith, EH, Bruce, H, 3rd: Proteinuria and hematuria in schoolchildren: epidemiology and early natural history. J Pediatr, 88: 327-347, 1976.

17. Ritchie, CD, Bevan, EA, Collier, SJ: Importance of occult haematuria found at screening. Br Med J (Clin Res Ed), 292: 681-683, 1986.

18. Dische, FE, Anderson, VE, Keane, SJ, Taube, D, Bewick, M, Parsons, V: Incidence of thin membrane nephropathy: morphometric investigation of a population sample. J Clin Pathol, 43: 457-460, 1990.

19. Savige, J, Gregory, M, Gross, O, Kashtan, C, Ding, J, Flinter, F: Expert guidelines for the management of Alport syndrome and thin basement membrane nephropathy. J Am Soc Nephrol, 24: 364-375, 2013.

20. Jais, JP, Knebelmann, B, Giatras, I, De Marchi, M, Rizzoni, G, Renieri, A, Weber, M, Gross, O, Netzer, KO, Flinter, F, Pirson, Y, Verellen, C, Wieslander, J, Persson, U, Tryggvason, K, Martin, P, Hertz, JM, Schroder, C, Sanak, M, Krejcova, S, Carvalho, MF, Saus, J, Antignac, C, Smeets, H, Gubler, MC: Xlinked Alport syndrome: natural history in 195 families and genotype- phenotype correlations in males. $J$ Am Soc Nephrol, 11: 649-657, 2000.

21. Jais, JP, Knebelmann, B, Giatras, I, De Marchi, M, Rizzoni, G, Renieri, A, Weber, M, Gross, O, Netzer, KO, Flinter, F, Pirson, Y, Dahan, K, Wieslander, J, Persson, U, Tryggvason, K, Martin, P, Hertz, JM, Schroder, C, Sanak, M, Carvalho, MF, Saus, J, Antignac, C, Smeets, H, Gubler, MC: X-linked Alport syndrome: natural history and genotype-phenotype correlations in girls and women belonging to 195 families: a "European Community Alport Syndrome Concerted Action" study. J Am Soc Nephrol, 14: 2603-2610, 2003.

22. Savige, J, Colville, D, Rheault, M, Gear, S, Lennon, R, Lagas, S, Finlay, M, Flinter, F: Alport Syndrome in Women and Girls. Clin J Am Soc Nephrol, 11: 1713-1720, 2016. 
23. Savige, J, Ariani, F, Mari, F, Bruttini, M, Renieri, A, Gross, O, Deltas, C, Flinter, F, Ding, J, Gale, DP, Nagel, M, Yau, M, Shagam, L, Torra, R, Ars, E, Hoefele, J, Garosi, G, Storey, H: Expert consensus guidelines for the genetic diagnosis of Alport syndrome. Pediatr Nephrol, 2018.

24. Richards, S, Aziz, N, Bale, S, Bick, D, Das, S, Gastier-Foster, J, Grody, WW, Hegde, M, Lyon, E, Spector, E, Voelkerding, K, Rehm, HL, Committee, ALQA: Standards and guidelines for the interpretation of sequence variants: a joint consensus recommendation of the American College of Medical Genetics and Genomics and the Association for Molecular Pathology. Genet Med, 17: 405-424, 2015.

25. Myllyharju, J, Kivirikko, KI: Collagens and collagen-related diseases. Ann Med, 33: 7-21, 2001.

26. Savige, J, Storey, H, Watson, E, Hertz, JM, Deltas, C, Renieri, A, Mari, F, Hilbert, P, Plevova, P, Byers, P, Cerkauskaite, A, Gregory, M, Cerkauskiene, R, Ljubanovic, DG, Becherucci, F, Errichiello, C, Massella, L, Aiello, V, Lennon, R, Hopkinson, L, Koziell, A, Lungu, A, Rothe, HM, Hoefele, J, Zacchia, M, Martic, TN, Gupta, A, van Eerde, A, Gear, S, Landini, S, Palazzo, V, Al-Rabadi, L, Claes, K, Corveleyn, A, Van Hoof, E, van Geel, M, Williams, M, Ashton, E, Belge, H, Ars, E, Bierzynska, A, Gangemi, C, LipskaZietkiewicz, BS: Consensus statement on standards and guidelines for the molecular diagnostics of Alport syndrome: refining the ACMG criteria. Eur J Hum Genet, 2021.

27. Mariyama, M, Leinonen, A, Mochizuki, T, Tryggvason, K, Reeders, ST: Complete primary structure of the human alpha 3(IV) collagen chain. Coexpression of the alpha 3(IV) and alpha 4(IV) collagen chains in human tissues. J Biol Chem, 269: 23013-23017, 1994.

28. Leinonen, A, Mariyama, M, Mochizuki, T, Tryggvason, K, Reeders, ST: Complete primary structure of the human type IV collagen alpha 4(IV) chain. Comparison with structure and expression of the other alpha (IV) chains. J Biol Chem, 269: 26172-26177, 1994.

29. Zhou, J, Hertz, JM, Leinonen, A, Tryggvason, K: Complete amino acid sequence of the human alpha 5 (IV) collagen chain and identification of a single-base mutation in exon 23 converting glycine 521 in the collagenous domain to cysteine in an Alport syndrome patient. J Biol Chem, 267: 12475-12481, 1992.

30. Demosthenous, P, Voskarides, K, Stylianou, K, Hadjigavriel, M, Arsali, M, Patsias, C, Georgaki, E, Zirogiannis, P, Stavrou, C, Daphnis, E, Pierides, A, Deltas, C, Hellenic Nephrogenetics Research, C: Xlinked Alport syndrome in Hellenic families: phenotypic heterogeneity and mutations near interruptions of the collagen domain in COL4A5. Clin Genet, 81: 240-248, 2012.

31. Lanktree, MB, Haghighi, A, Guiard, E, lliuta, IA, Song, X, Harris, PC, Paterson, AD, Pei, Y: Prevalence Estimates of Polycystic Kidney and Liver Disease by Population Sequencing. J Am Soc Nephrol, 29: 2593-2600, 2018.

32. Lek, M, Karczewski, KJ, Minikel, EV, Samocha, KE, Banks, E, Fennell, T, O'Donnell-Luria, AH, Ware, JS, Hill, AJ, Cummings, BB, Tukiainen, T, Birnbaum, DP, Kosmicki, JA, Duncan, LE, Estrada, K, Zhao, F, Zou, J, Pierce-Hoffman, E, Berghout, J, Cooper, DN, Deflaux, N, DePristo, M, Do, R, Flannick, J, Fromer, M, Gauthier, L, Goldstein, J, Gupta, N, Howrigan, D, Kiezun, A, Kurki, MI, Moonshine, AL, Natarajan, P, Orozco, L, Peloso, GM, Poplin, R, Rivas, MA, Ruano-Rubio, V, Rose, SA, Ruderfer, DM, Shakir, K, Stenson, PD, Stevens, C, Thomas, BP, Tiao, G, Tusie-Luna, MT, Weisburd, B, Won, HH, Yu, D, Altshuler, DM, Ardissino, D, Boehnke, M, Danesh, J, Donnelly, S, Elosua, R, Florez, JC, Gabriel, SB, Getz, G, Glatt, SJ, Hultman, CM, Kathiresan, S, Laakso, M, McCarroll, S, McCarthy, MI, McGovern, D, McPherson, R, Neale, BM, Palotie, A, Purcell, SM, Saleheen, D, Scharf, JM, Sklar, P, Sullivan, PF, Tuomilehto, J, Tsuang, MT, Watkins, HC, Wilson, JG, Daly, MJ, MacArthur, DG, Exome Aggregation, C: Analysis of protein-coding genetic variation in 60,706 humans. Nature, 536: 285-291, 2016.

33. The National Genomics Research and Healthcare Knowledgebase v5, Genomics England. 5, 2019.

34. van der Ven, AT, Connaughton, DM, Ityel, H, Mann, N, Nakayama, M, Chen, J, Vivante, A, Hwang, DY, Schulz, J, Braun, DA, Schmidt, JM, Schapiro, D, Schneider, R, Warejko, JK, Daga, A, Majmundar, AJ, Tan, W, Jobst-Schwan, T, Hermle, T, Widmeier, E, Ashraf, S, Amar, A, Hoogstraaten, CA, Hugo, H, Kitzler, TM, Kause, F, Kolvenbach, CM, Dai, R, Spaneas, L, Amann, K, Stein, DR, Baum, MA, Somers, MJG, Rodig, NM, Ferguson, MA, Traum, AZ, Daouk, GH, Bogdanovic, R, Stajic, N, Soliman, NA, Kari, JA, El Desoky, S, Fathy, HM, Milosevic, D, Al-Saffar, M, Awad, HS, Eid, LA, Selvin, A, Senguttuvan, P, Sanna-Cherchi, S, Rehm, HL, MacArthur, DG, Lek, M, Laricchia, KM, Wilson, MW, Mane, SM, Lifton, RP, Lee, RS, Bauer, SB, Lu, W, Reutter, HM, Tasic, V, Shril, S, Hildebrandt, F: Whole-Exome Sequencing Identifies Causative Mutations in Families with Congenital Anomalies of the Kidney and Urinary Tract. $J$ Am Soc Nephrol, 29: 2348-2361, 2018.

35. Kelly, MA, Caleshu, C, Morales, A, Buchan, J, Wolf, Z, Harrison, SM, Cook, S, Dillon, MW, Garcia, J, Haverfield, E, Jongbloed, JDH, Macaya, D, Manrai, A, Orland, K, Richard, G, Spoonamore, K, Thomas, M, Thomson, K, Vincent, LM, Walsh, R, Watkins, H, Whiffin, N, Ingles, J, van Tintelen, JP, Semsarian, C, Ware, JS, Hershberger, R, Funke, B: Adaptation and validation of the ACMG/AMP variant classification framework for MYH7-associated inherited cardiomyopathies: recommendations by ClinGen's Inherited Cardiomyopathy Expert Panel. Genet Med, 20: 351-359, 2018. 
36. Biesecker, LG, Harrison, SM, ClinGen Sequence Variant Interpretation Working, G: The ACMG/AMP reputable source criteria for the interpretation of sequence variants. Genet Med, 20: 1687-1688, 2018.

37. Richards, CS, Aziz, N, Bale, S, Bick, D, Das, S, Gastier-Foster, J, Grody, WW, Hegde, M, Lyon, E, Spector, E, Voelkerding, K, Rehm, HL, Group, AAloSVW: Response to Biesecker and Harrison. Genet Med, 20: 1689-1690, 2018.

38. Abou Tayoun, AN, Pesaran, T, DiStefano, MT, Oza, A, Rehm, HL, Biesecker, LG, Harrison, SM, ClinGen Sequence Variant Interpretation Working, G: Recommendations for interpreting the loss of function PVS1 ACMG/AMP variant criterion. Hum Mutat, 39: 1517-1524, 2018.

39. Hashimura, Y, Nozu, K, Kaito, H, Nakanishi, K, Fu, XJ, Ohtsubo, H, Hashimoto, F, Oka, M, Ninchoji, T, Ishimori, S, Morisada, N, Matsunoshita, N, Kamiyoshi, N, Yoshikawa, N, lijima, K: Milder clinical aspects of X-linked Alport syndrome in men positive for the collagen IV alpha5 chain. Kidney Int, 85: 1208-1213, 2014.

40. Savige, J, Storey, H, Il Cheong, H, Gyung Kang, H, Park, E, Hilbert, P, Persikov, A, Torres-Fernandez, C, Ars, E, Torra, R, Hertz, JM, Thomassen, M, Shagam, L, Wang, D, Wang, Y, Flinter, F, Nagel, M: X-Linked and Autosomal Recessive Alport Syndrome: Pathogenic Variant Features and Further Genotype-Phenotype Correlations. PLoS One, 11: e0161802, 2016.

41. Persikov, AV, Pillitteri, RJ, Amin, P, Schwarze, U, Byers, PH, Brodsky, B: Stability related bias in residues replacing glycines within the collagen triple helix (Gly-Xaa-Yaa) in inherited connective tissue disorders. Hum Mutat, 24: 330-337, 2004.

42. Storey, H, Savige, J, Sivakumar, V, Abbs, S, Flinter, FA: COL4A3/COL4A4 mutations and features in individuals with autosomal recessive Alport syndrome. J Am Soc Nephrol, 24: 1945-1954, 2013.

43. Slajpah, M, Gorinsek, B, Berginc, G, Vizjak, A, Ferluga, D, Hvala, A, Meglic, A, Jaksa, I, Furlan, P, Gregoric, A, Kaplan-Pavlovcic, S, Ravnik-Glavac, M, Glavac, D: Sixteen novel mutations identified in COL4A3, COL4A4, and COL4A5 genes in Slovenian families with Alport syndrome and benign familial hematuria. Kidney Int, 71: 1287-1295, 2007.

44. Macheroux, EP, Braunisch, MC, Pucci Pegler, S, Satanovskij, R, Riedhammer, KM, Gunthner, R, Gross, O, Nagel, M, Renders, L, Hoefele, J: The Hypomorphic Variant p.(Gly624Asp) in COL4A5 as a Possible Cause for an Unexpected Severe Phenotype in a Family With X-Linked Alport Syndrome. Front Pediatr, 7: 485, 2019.

45. Zurowska, AM, Bielska, O, Daca-Roszak, P, Jankowski, M, Szczepanska, M, Roszkowska-Bjanid, D, KuzmaMroczkowska, E, Panczyk-Tomaszewska, M, Moczulska, A, Drozdz, D, Hadjipanagi, D, Deltas, C, Ostalska-Nowicka, D, Rabiega, A, Taraszkiewicz, J, Taranta-Janusz, K, Wieczorkiewicz-Plaza, A, Jobs, K, Mews, J, Musial, K, Jakubowska, A, Nosek, H, Jander, AE, Koutsofti, C, Stanislawska-Sachadyn, A, Kuleszo, D, Zietkiewicz, E, Lipska-Zietkiewicz, BS: Mild X-linked Alport syndrome due to the COL4A5 G624D variant originating in the Middle Ages is predominant in Central/East Europe and causes kidney failure in midlife. Kidney Int, 2020.

46. AM, Z: Mild X-linked Alport syndrome due to the COL4A5 G624D variant origtinating in the Middle Ages is predominant in Central/East Europe and causes kidney failure in mid-life. Kidney Int, 2021.

47. Feingold, J, Bois, E, Chompret, A, Broyer, M, Gubler, MC, Grunfeld, JP: Genetic heterogeneity of Alport syndrome. Kidney Int, 27: 672-677, 1985.

48. Lemmink, HH, Nillesen, WN, Mochizuki, T, Schroder, CH, Brunner, HG, van Oost, BA, Monnens, LA, Smeets, $\mathrm{HJ}$ : Benign familial hematuria due to mutation of the type IV collagen alpha4 gene. J Clin Invest, 98 : 1114-1118, 1996.

49. Persikov, AV, Ramshaw, JA, Kirkpatrick, A, Brodsky, B: Amino acid propensities for the collagen triple-helix. Biochemistry, 39: 14960-14967, 2000.

50. Fallerini, C, Baldassarri, M, Trevisson, E, Morbidoni, V, La Manna, A, Lazzarin, R, Pasini, A, Barbano, G, Pinciaroli, AR, Garosi, G, Frullanti, E, Pinto, AM, Mencarelli, MA, Mari, F, Renieri, A, Ariani, F: Alport syndrome: impact of digenic inheritance in patients management. Clin Genet, 92: 34-44, 2017.

51. Makareeva, E, Mertz, EL, Kuznetsova, NV, Sutter, MB, DeRidder, AM, Cabral, WA, Barnes, AM, McBride, DJ, Marini, JC, Leikin, S: Structural heterogeneity of type I collagen triple helix and its role in osteogenesis imperfecta. J Biol Chem, 283: 4787-4798, 2008.

52. Gross, O, Licht, C, Anders, HJ, Hoppe, B, Beck, B, Tonshoff, B, Hocker, B, Wygoda, S, Ehrich, JH, Pape, L, Konrad, M, Rascher, W, Dotsch, J, Muller-Wiefel, DE, Hoyer, P, Study Group Members of the Gesellschaft fur Padiatrische, N, Knebelmann, B, Pirson, Y, Grunfeld, JP, Niaudet, P, Cochat, P, Heidet, L, Lebbah, S, Torra, R, Friede, T, Lange, K, Muller, GA, Weber, M: Early angiotensin-converting enzyme inhibition in Alport syndrome delays renal failure and improves life expectancy. Kidney Int, 81: 494-501, 2012.

53. Kaariainen, H, Muilu, J, Perola, M, Kristiansson, K: Genetics in an isolated population like Finland: a different basis for genomic medicine? J Community Genet, 8: 319-326, 2017.

54. Kashtan, CE: Alport Syndrome. Gene Reviews 
In: Adam MP, Ardinger HH, Pagon RA, et al., editors. GeneReviews ${ }^{\circledR}$ [Internet]. Seattle (WA): University of Washington, Seattle; 1993-2020. Available from: https://www.ncbi.nlm.nih.gov/books/NBK1207/. 2001.

55. Pierides, A, Voskarides, K, Athanasiou, Y, loannou, K, Damianou, L, Arsali, M, Zavros, M, Pierides, M, Vargemezis, V, Patsias, C, Zouvani, I, Elia, A, Kyriacou, K, Deltas, C: Clinico-pathological correlations in 127 patients in 11 large pedigrees, segregating one of three heterozygous mutations in the COL4A3/ COL4A4 genes associated with familial haematuria and significant late progression to proteinuria and chronic kidney disease from focal segmental glomerulosclerosis. Nephrol Dial Transplant, 24: 2721-2729, 2009.

56. Matthaiou, M, Poulii, T, Matthaiou, M, Matthaiou, M, Poulli, T, Deltas, C: Prevalence of clinical, pathological and molecular features of glomerular basement membrane nephropathy caused by COL4A3 or COL4A4 mutations: a systematic review. Clinical Kidney Journal 1-12, 2020.

57. Gillion, V, Dahan, K, Cosyns, JP, Hilbert, P, Jadoul, M, Goffin, E, Godefroid, N, De Meyer, M, Mourad, M, Pirson, Y, Kanaan, N: Genotype and Outcome After Kidney Transplantation in Alport Syndrome. Kidney Int Rep, 3: 652-660, 2018.

58. Groopman, EE, Marasa, M, Cameron-Christie, S, Petrovski, S, Aggarwal, VS, Milo-Rasouly, H, Li, Y, Zhang, J, Nestor, J, Krithivasan, P, Lam, WY, Mitrotti, A, Piva, S, Kil, BH, Chatterjee, D, Reingold, R, Bradbury, D, DiVecchia, M, Snyder, H, Mu, X, Mehl, K, Balderes, O, Fasel, DA, Weng, C, Radhakrishnan, J, Canetta, P, Appel, GB, Bomback, AS, Ahn, W, Uy, NS, Alam, S, Cohen, DJ, Crew, RJ, Dube, GK, Rao, MK, Kamalakaran, S, Copeland, B, Ren, Z, Bridgers, J, Malone, CD, Mebane, CM, Dagaonkar, N, Fellstrom, BC, Haefliger, C, Mohan, S, Sanna-Cherchi, S, Kiryluk, K, Fleckner, J, March, R, Platt, A, Goldstein, DB, Gharavi, AG: Diagnostic Utility of Exome Sequencing for Kidney Disease. N Engl J Med, 380: 142-151, 2019.

59. Fallerini, C, Dosa, L, Tita, R, Del Prete, D, Feriozzi, S, Gai, G, Clementi, M, La Manna, A, Miglietti, N, Mancini, R, Mandrile, G, Ghiggeri, GM, Piaggio, G, Brancati, F, Diano, L, Frate, E, Pinciaroli, AR, Giani, M, Castorina, P, Bresin, E, Giachino, D, De Marchi, M, Mari, F, Bruttini, M, Renieri, A, Ariani, F: Unbiased next generation sequencing analysis confirms the existence of autosomal dominant Alport syndrome in a relevant fraction of cases. Clin Genet, 86: 252-257, 2014.

60. Bullich, G, Domingo-Gallego, A, Vargas, I, Ruiz, P, Lorente-Grandoso, L, Furlano, M, Fraga, G, Madrid, A, Ariceta, G, Borregan, M, Pinero-Fernandez, JA, Rodriguez-Pena, L, Ballesta-Martinez, MJ, Llano-Rivas, I, Menica, MA, Ballarin, J, Torrents, D, Torra, R, Ars, E: A kidney-disease gene panel allows a comprehensive genetic diagnosis of cystic and glomerular inherited kidney diseases. Kidney Int, 94: 363371, 2018.

61. Gross, O, Tonshoff, B, Weber, LT, Pape, L, Latta, K, Fehrenbach, H, Lange-Sperandio, B, Zappel, H, Hoyer, P, Staude, H, Konig, S, John, U, Gellermann, J, Hoppe, B, Galiano, M, Hoecker, B, Ehren, R, Lerch, C, Kashtan, CE, Harden, M, Boeckhaus, J, Friede, T, German Pediatric Nephrology Study, G, Investigators, EP-TA: A multicenter, randomized, placebo-controlled, double-blind phase 3 trial with open-arm comparison indicates safety and efficacy of nephroprotective therapy with ramipril in children with Alport's syndrome. Kidney Int, 97: 1275-1286, 2020.

62. Hertz, JM, Juncker, I, Persson, U, Matthijs, G, Schmidtke, J, Petersen, MB, Kjeldsen, M, Gregersen, N: Detection of mutations in the COL4A5 gene by SSCP in X-linked Alport syndrome. Hum Mutat, 18: 141148, 2001.

63. Plant, KE, Green, PM, Vetrie, D, Flinter, FA: Detection of mutations in COL4A5 in patients with Alport syndrome. Hum Mutat, 13: 124-132, 1999.

64. King, K, Flinter, FA, Green, PM: A two-tier approach to mutation detection in the COL4A5 gene for Alport syndrome. Hum Mutat, 27: 1061, 2006.

65. Cruz-Robles, D, Garcia-Torres, R, Antignac, C, Forestier, L, de la Puente, SG, Correa-Rotter, R, GarciaLopez, E, Orozco, L: Three novel mutations in the COL4A5 gene in Mexican Alport syndrome patients. Clin Genet, 56: 242-243, 1999.

66. Bekheirnia, MR, Reed, B, Gregory, MC, McFann, K, Shamshirsaz, AA, Masoumi, A, Schrier, RW: Genotypephenotype correlation in X-linked Alport syndrome. J Am Soc Nephrol, 21: 876-883, 2010.

67. Mohammad, M, Nanra, R, Colville, D, Trevillian, P, Wang, Y, Storey, H, Flinter, F, Savige, J: A female with Xlinked Alport syndrome and compound heterozygous COL4A5 mutations. Pediatr Nephrol, 29: 481-485, 2014.

68. Knebelmann, B, Breillat, C, Forestier, L, Arrondel, C, Jacassier, D, Giatras, I, Drouot, L, Deschenes, G, Grunfeld, JP, Broyer, M, Gubler, MC, Antignac, C: Spectrum of mutations in the COL4A5 collagen gene in X-linked Alport syndrome. Am J Hum Genet, 59: 1221-1232, 1996.

69. Inoue, Y, Nishio, H, Shirakawa, T, Nakanishi, K, Nakamura, H, Sumino, K, Nishiyama, K, lijima, K, Yoshikawa, N: Detection of mutations in the COL4A5 gene in over $90 \%$ of male patients with $\mathrm{X}$-linked Alport's syndrome by RT-PCR and direct sequencing. Am J Kidney Dis, 34: 854-862, 1999. 
Copyright 2021 by ASN, Published Ahead of Print on 6/18/21, Accepted/Unedited Version

70. Zhang, H, Ding, J, Wang, F, Zhao, D: Mutation detection of COL4An gene based on mRNA of peripheral blood lymphocytes and prenatal diagnosis of Alport syndrome in China. Nephrology (Carlton), 16: 377380, 2011. 
Copyright 2021 by ASN, Published Ahead of Print on 6/18/21, Accepted/Unedited Version

Table 1: Association of predicted pathogenic COL4A3 - COL4A5 variants resulting in position 1 Gly substitutions with haematuria in 100,000 Genomes Project database

\begin{tabular}{|c|c|c|c|c|c|}
\hline \multirow[t]{2}{*}{ Gene } & \multirow{2}{*}{$\begin{array}{l}\text { Number of } \\
\text { variants } \\
\text { resulting in } \\
\text { position } 1 \text { Gly } \\
\text { substitutions in } \\
100,000 \\
\text { Genomes } \\
\text { Project }\end{array}$} & \multirow{2}{*}{$\begin{array}{l}\text { Number of } \\
\text { variants } \\
\text { resulting in } \\
\text { position } 1 \text { Gly } \\
\text { substitutions } \\
\text { excluded after } \\
\text { filtering }\end{array}$} & \multicolumn{2}{|c|}{$\begin{array}{l}\text { Number of individuals with } \\
\text { a predicted pathogenic } \\
\text { position } 1 \text { Gly substitution }\end{array}$} & \multirow[t]{2}{*}{$\begin{array}{l}\text { Chi-square (with } \\
\text { Yate's correction), } \\
\text { two-tailed p value }\end{array}$} \\
\hline & & & $\begin{array}{l}\text { In total with } \\
\text { haematuria } \\
(n=2,221)\end{array}$ & $\begin{array}{l}\text { In total } \\
\text { without } \\
\text { haematuria } \\
(n=37,200)\end{array}$ & \\
\hline COL4A5 & 20 & $1(5 \%)$ & 12 & 9 & $95.83, p<0.0001$ \\
\hline COL4A3 & 87 & $14(16 \%)$ & 21 & 85 & $37.54, p<0.0001$ \\
\hline COL4A4 & 84 & $13(15 \%)$ & 19 & 95 & $24.14, p<0.0001$ \\
\hline
\end{tabular}


Copyright 2021 by ASN, Published Ahead of Print on 6/18/21, Accepted/Unedited Version

Table 2: Assessment of COL4A5 variants in the gnomAD database and gnomAD control subset

\begin{tabular}{|c|c|c|c|c|c|c|c|c|c|c|c|c|}
\hline \multicolumn{11}{|c|}{ Total gnomAD database } & \multicolumn{2}{|c|}{$\begin{array}{c}\text { gnomAD control } \\
\text { subset }\end{array}$} \\
\hline $\begin{array}{c}\text { Position } \\
\text { hg19 and } \\
\text { Protein } \\
\text { consequenc } \\
\text { e }\end{array}$ & $\underset{\text { pt }}{\text { Transcri }}$ & $\begin{array}{l}\text { Affected } \\
\text { individual } \\
\text { s gender, } \\
\text { total } \\
\text { alleles }\end{array}$ & PP2 & SIFT & MT & $\begin{array}{c}\text { Conserve } \\
\mathrm{d}\end{array}$ & $\begin{array}{l}\text { Previously } \\
\text { reported; } \\
\text { alternative } \\
\text { pathogenic } \\
\text { residue } \\
\text { (LOVD) }\end{array}$ & $\begin{array}{l}\text { Clin } \\
\text { Var }\end{array}$ & $\begin{array}{l}\text { Pathogenicity } \\
\text { (Varsome) }\end{array}$ & $\begin{array}{l}\text { Patho- } \\
\text { genicity } \\
\text { (Alamut) }\end{array}$ & $\begin{array}{l}\text { Evidenc } \\
\text { e } \\
\text { support } \\
\text { s } \\
\text { patho- } \\
\text { genicity }\end{array}$ & $\begin{array}{c}\text { Affected } \\
\text { individuals } \\
\text { gender, total } \\
\text { alleles }\end{array}$ \\
\hline $\begin{array}{l}107783018 \\
\text { p.(Gly42Ser) }\end{array}$ & $\begin{array}{l}\text { C. } 124 G> \\
\text { A }\end{array}$ & $\begin{array}{c}1 \mathrm{M} \\
21,780\end{array}$ & 0 & Tol & DC & Gly & NO & $\begin{array}{l}\text { Not } \\
\text { found }\end{array}$ & $\begin{array}{c}\text { Likely pathogenic, } \\
\text { PM1, } \\
\text { PM2, PP2,PP3 }\end{array}$ & Weak & NO & \\
\hline $\begin{array}{l}107783019 \\
\text { p.(Gly42Ala) }\end{array}$ & $\begin{array}{l}\text { c. } 125 G> \\
\text { C }\end{array}$ & $\begin{array}{c}1 \mathrm{~F} \\
182,802\end{array}$ & 0.003 & Tol & DC & Gly & NO & $\begin{array}{l}\text { Not } \\
\text { found }\end{array}$ & $\begin{array}{l}\text { Likely pathogenic, } \\
\text { PM1,PM2,PP2,PP } \\
3\end{array}$ & Weak & $\mathrm{NO}$ & $\begin{array}{c}1 \mathrm{~F} \\
80,278\end{array}$ \\
\hline $\begin{array}{l}107783019 \\
\text { p.(Gly42Asp) }\end{array}$ & $\begin{array}{l}\text { C. } 125 G> \\
\text { A }\end{array}$ & $\begin{array}{c}1 \mathrm{~F} \\
182,802\end{array}$ & 0.739 & Tol & DC & Gly & NO & $\begin{array}{l}\text { Not } \\
\text { found }\end{array}$ & $\begin{array}{l}\text { Likely pathogenic, } \\
\text { PM1,PM2,PP2,PP } \\
3\end{array}$ & Weak & $\mathrm{NO}$ & \\
\hline $\begin{array}{l}107802357 \\
\text { p.(Gly69Arg) }\end{array}$ & $\begin{array}{l}\text { c. } 205 G> \\
\text { C }\end{array}$ & $\begin{array}{c}1 \mathrm{~F} \\
183,072\end{array}$ & 0.01 & Del & DC & Gly & NO & $\begin{array}{l}\text { Not } \\
\text { found }\end{array}$ & $\begin{array}{l}\text { Likely pathogenic, } \\
\text { PM1,PM2,PP2,PP } \\
3\end{array}$ & Strong & YES (1F) & \\
\hline $\begin{array}{l}107807131 \\
\text { p.(Gly84Glu) }\end{array}$ & $\begin{array}{l}\text { C. } 251 G> \\
\text { A }\end{array}$ & $\begin{array}{c}1 \mathrm{~F} \\
182,367\end{array}$ & 1 & Del & DC & Gly & NO & $\begin{array}{l}\text { Not } \\
\text { found }\end{array}$ & $\begin{array}{c}\text { Likely pathogenic, } \\
\text { PM1,PM2,PM5,PP } \\
2, \\
\text { PP3 }\end{array}$ & Strong & $\begin{array}{l}\text { YES } \\
(1 F)\end{array}$ & \\
\hline $\begin{array}{l}107811896 \\
\text { p.(Gly105Ala) }\end{array}$ & $\begin{array}{l}\text { c. } 314 G> \\
\text { C }\end{array}$ & $\begin{array}{c}1 \mathrm{M} \\
183,404\end{array}$ & 1 & Del & DC & Gly & NO & $\begin{array}{l}\text { Not } \\
\text { found }\end{array}$ & $\begin{array}{c}\text { Likely pathogenic, } \\
\text { PM1,PM2,PP2,PP } \\
3\end{array}$ & $\begin{array}{c}\text { Moderat } \\
\text { e }\end{array}$ & $\begin{array}{l}\text { YES } \\
(1 \mathrm{M})\end{array}$ & $\begin{array}{c}1 \mathrm{M} \\
80,333\end{array}$ \\
\hline $\begin{array}{l}107812008 \\
\text { p.(Gly114Ala) }\end{array}$ & $\begin{array}{l}\text { c. } 341 G> \\
C\end{array}$ & $\begin{array}{c}1 \mathrm{~F} \\
183,513\end{array}$ & 1 & Del & DC & Gly & $\begin{array}{l}\text { NO, but } \\
\text { p.(Gly114Arg) } \\
\text { reported in } \\
\text { LOVD } \\
\end{array}$ & $\begin{array}{l}\text { Not } \\
\text { found }\end{array}$ & $\begin{array}{c}\text { Likely pathogenic, } \\
\text { PM1,PM2,PM5,PP } \\
2, \\
\text { PP3 }\end{array}$ & $\begin{array}{c}\text { Moderat } \\
\mathrm{e}\end{array}$ & $\begin{array}{l}\text { YES } \\
(1 F)\end{array}$ & $\begin{array}{c}1 \mathrm{~F} \\
80,345\end{array}$ \\
\hline $\begin{array}{l}107812044 \\
\text { p.(Gly126Glu) }\end{array}$ & $\begin{array}{l}\text { C. } 377 G> \\
\text { A }\end{array}$ & $\begin{array}{c}1 \mathrm{~F} \\
21,871\end{array}$ & 1 & Del & DC & Gly & NO & $\begin{array}{l}\text { Not } \\
\text { found }\end{array}$ & $\begin{array}{l}\text { Likely pathogenic, } \\
\text { PM1,PM2,PP2,PP } \\
3\end{array}$ & $\begin{array}{c}\text { Moderat } \\
\text { e }\end{array}$ & $\begin{array}{l}\text { YES } \\
(1 F)\end{array}$ & $\begin{array}{c}1 \mathrm{~F} \\
7600\end{array}$ \\
\hline $\begin{array}{l}107814670 \\
\text { p.(Gly138Ser) }\end{array}$ & $\begin{array}{l}\text { C.412G> } \\
\text { A }\end{array}$ & $\begin{array}{c}1 \mathrm{~F} \\
183,271\end{array}$ & 1 & Tol & DC & Gly & $\begin{array}{c}\text { YES } \\
\text { Fallerini, } 2014 \\
\text { 59; and } \\
\text { p(Gly138Asp) }\end{array}$ & $\begin{array}{l}\text { Pathog } \\
\text { enic } \\
\text { (no } \\
\text { asserti }\end{array}$ & $\begin{array}{c}\text { Likely pathogenic, } \\
\text { PM1, } \\
\text { PM2,PM5,PP2,PP } \\
\text { 3, PP\% }\end{array}$ & Weak & NO & \\
\hline
\end{tabular}


Copyright 2021 by ASN, Published Ahead of Print on 6/18/21, Accepted/Unedited Version

\begin{tabular}{|c|c|c|c|c|c|c|c|c|c|c|c|c|}
\hline & & & & & & & in LOVD & $\begin{array}{c}\text { on } \\
\text { criteria) }\end{array}$ & & & & \\
\hline $\begin{array}{l}107819195 \\
\text { p.(Gly201Ala) }\end{array}$ & $\begin{array}{l}\text { c. } 602 G> \\
\text { C }\end{array}$ & $\begin{array}{c}1 \mathrm{M} \\
168,800\end{array}$ & 0.999 & Del & DC & Gly & \begin{tabular}{|c|} 
NO, but \\
p(Gly201Val), \\
Hertz 2001 62 \\
\end{tabular} & $\begin{array}{c}\text { Not } \\
\text { found }\end{array}$ & $\begin{array}{c}\text { Likely pathogenic, } \\
\text { PM1,PM2,PM5,PP } \\
\text { 2,PP3 }\end{array}$ & $\begin{array}{c}\text { Moderat } \\
\text { e }\end{array}$ & $\begin{array}{l}\text { YES } \\
(1 \mathrm{M})\end{array}$ & \\
\hline $\begin{array}{l}107823808 \\
\text { p.(Gly276Ser }\end{array}$ & $\begin{array}{l}\text { c. } 826 G> \\
A\end{array}$ & $\begin{array}{c}1 \mathrm{~F}, \\
183,230\end{array}$ & 1 & Tol & DC & Gly & NO & $\begin{array}{l}\text { Not } \\
\text { found }\end{array}$ & $\begin{array}{l}\text { Likely pathogenic, } \\
\text { PM1,PM2,PP2,PP } \\
3\end{array}$ & Weak & & $\begin{array}{c}1 \mathrm{~F} \\
80,319\end{array}$ \\
\hline $\begin{array}{l}107823912 \\
\text { p.(Gly279Arg } \\
\text { ) }\end{array}$ & $\begin{array}{l}\text { c. } 835 G> \\
C\end{array}$ & $\begin{array}{c}1 \mathrm{M}, \\
183,017\end{array}$ & 1 & Tol & DC & Gly & NO & $\begin{array}{l}\text { Not } \\
\text { found }\end{array}$ & $\begin{array}{l}\text { Likely pathogenic, } \\
\text { PM1,PM2,PP2,PP } \\
3 \\
\end{array}$ & $\begin{array}{l}\text { Moderat } \\
\text { e }\end{array}$ & $\mathrm{NO}$ & \\
\hline $\begin{array}{l}107823913 \\
\text { p.(Gly279Ala) }\end{array}$ & $\begin{array}{l}\text { c. } 836 G> \\
\text { C }\end{array}$ & $\begin{array}{c}1 \mathrm{M} \\
183,034\end{array}$ & 1 & Del & DC & Gly & NO & $\begin{array}{l}\text { Not } \\
\text { found }\end{array}$ & $\begin{array}{l}\text { Likely pathogenic, } \\
\text { PM1,PM2,PP2,PP } \\
3\end{array}$ & Weak & $\begin{array}{l}\text { YES } \\
(1 \mathrm{M})\end{array}$ & $\begin{array}{c}1 \mathrm{M} \\
80,323\end{array}$ \\
\hline p.(Gly283Val) & $\begin{array}{l}\text { c.848G> } \\
T\end{array}$ & $\begin{array}{c}1 \mathrm{~F} \\
183,010\end{array}$ & 1 & Del & DC & Gly & NO & $\begin{array}{l}\text { Not } \\
\text { found }\end{array}$ & $\begin{array}{l}\text { Likely pathogenic, } \\
\text { PM1,PM2,PP2,PP } \\
3\end{array}$ & Strong & $\begin{array}{l}\text { YES } \\
(1 F)\end{array}$ & \\
\hline p.(Gly286Cys & $\begin{array}{l}\text { c. } 856 \mathrm{G}> \\
\mathrm{T}\end{array}$ & $\begin{array}{c}1 \mathrm{~F} \\
182,986\end{array}$ & 1 & Del & DC & Gly & $\mathrm{NO}$ & $\begin{array}{l}\text { Not } \\
\text { found }\end{array}$ & $\begin{array}{l}\text { Likely pathogenic, } \\
\text { PM1,PM2,PP2,PP } \\
3\end{array}$ & Strong & $\begin{array}{l}\text { YES } \\
(1 F)\end{array}$ & $\begin{array}{c}1 \mathrm{~F} \\
80,324\end{array}$ \\
\hline $\begin{array}{l}107829879 \\
\text { p.(Gly356Ala) }\end{array}$ & $\begin{array}{l}\text { c. } 1067 G \\
>C\end{array}$ & $\begin{array}{c}1 \mathrm{~F} \\
21,220\end{array}$ & 0.997 & Del & DC & Gly & NO & $\begin{array}{l}\text { Not } \\
\text { found }\end{array}$ & $\begin{array}{l}\text { Likely pathogenic, } \\
\text { PM1,PM2,PP2,PP } \\
3 \\
\end{array}$ & Strong & $\begin{array}{l}\text { YES } \\
(1 \mathrm{~F})\end{array}$ & \\
\hline $\begin{array}{l}107829879 \\
\text { p.(Gly356Glu }\end{array}$ & $\begin{array}{l}\text { c. } 1067 G \\
>A\end{array}$ & $\begin{array}{c}1 \mathrm{M} \\
183,308\end{array}$ & 0.99 & Del & DC & Gly & NO & $\begin{array}{l}\text { Not } \\
\text { found }\end{array}$ & $\begin{array}{l}\text { Likely pathogenic, } \\
\text { PM1,PM2,PP2,PP } \\
3 \\
\end{array}$ & Strong & $\begin{array}{l}\text { YES } \\
(1 \mathrm{M})\end{array}$ & \\
\hline $\begin{array}{l}\text { p.(Gly512Glu } \\
\text { ) }\end{array}$ & $\begin{array}{l}\text { c. } 1535 G \\
>A\end{array}$ & $\begin{array}{c}1 \mathrm{M} \\
145,435\end{array}$ & 1 & Del & DC & Gly & NO & $\begin{array}{l}\text { Not } \\
\text { found }\end{array}$ & $\begin{array}{l}\text { Likely pathogenic, } \\
\text { PM1,PM2,PP2,PP } \\
3 \\
\end{array}$ & Strong & $\begin{array}{l}\text { YES } \\
(1 \mathrm{M})\end{array}$ & \\
\hline $\begin{array}{l}107840625 \\
\text { p.(Gly536Ser }\end{array}$ & $\begin{array}{l}\text { c. } 1606 G \\
>A\end{array}$ & $\begin{array}{c}1 \mathrm{~F} \\
183,226\end{array}$ & 1 & Del & DC & Gly & $\begin{array}{c}\text { NO, but } \\
\text { p(Gly536Asp) } \\
\text { p(Gly536Val } \\
\text { ), in LOVD }\end{array}$ & $\begin{array}{l}\text { Not } \\
\text { found }\end{array}$ & $\begin{array}{c}\text { Likely pathogenic, } \\
\text { PM1,PM2, } \\
\text { PM5,PP2,PP3 }\end{array}$ & Strong & $\begin{array}{l}\text { YES } \\
(1 F)\end{array}$ & \\
\hline $\begin{array}{l}107844640 \\
\text { p.(Gly624Asp } \\
\text { ) }\end{array}$ & $\begin{array}{l}\text { c. } 1871 G \\
>A\end{array}$ & $\begin{array}{l}4 \mathrm{M}, 12 \mathrm{~F} \\
182,998\end{array}$ & 1 & Del & DC & Gly & $\begin{array}{l}\text { YES } \\
\text { Slajpah, } \\
2007^{43}\end{array}$ & $\begin{array}{l}\text { Pathog } \\
\text { enic ** }\end{array}$ & $\begin{array}{c}\text { Likely pathogenic } \\
\text { PM1,PP2,PP3 and } \\
\text { PP5 }\end{array}$ & Strong & $\begin{array}{l}\text { YES } \\
(4 \mathrm{M}, 12 \mathrm{~F}\end{array}$ & $\begin{array}{l}2 \mathrm{M}, 6 \mathrm{~F}, \\
80,268\end{array}$ \\
\hline $\begin{array}{l}107842028 \\
\text { p.(Gly626Ser } \\
\text { ) }\end{array}$ & $\begin{array}{l}\text { c. } 1876 G \\
>A\end{array}$ & $\begin{array}{c}2 \mathrm{M}, 5 \mathrm{~F} \\
204,674\end{array}$ & 1 & Del & DC & Gly & $\begin{array}{c}\text { YES Hertz, } \\
2001^{62}\end{array}$ & $\begin{array}{l}\text { Likely } \\
\text { benign } \\
\text { * }\end{array}$ & $\begin{array}{c}\text { Likely pathogenic, } \\
\text { PM1,PM2,PM5,PP } \\
\text { 2,PP3, BP6 }\end{array}$ & Strong & NO & $\begin{array}{l}1 \mathrm{M}, 4 \mathrm{~F}, \\
87,809\end{array}$ \\
\hline 107844640 & c. $1966 G$ & $1 \mathrm{M}$ & 1 & Del & DC & Gly & NO & Not & Likely pathogenic, & Strong & YES & \\
\hline
\end{tabular}


Copyright 2021 by ASN, Published Ahead of Print on 6/18/21, Accepted/Unedited Version

\begin{tabular}{|c|c|c|c|c|c|c|c|c|c|c|c|c|}
\hline $\begin{array}{l}\text { p.(Gly656Ser } \\
\text { ) }\end{array}$ & $>\mathrm{A}$ & 181,478 & & & & & & found & $\begin{array}{c}\text { PM1,PM2,PP2,PP } \\
3\end{array}$ & & $(1 \mathrm{M})$ & \\
\hline $\begin{array}{l}107845177 \\
\text { p.(Gly702Ser }\end{array}$ & $\begin{array}{l}\text { C. } 2104 G \\
>A\end{array}$ & $\begin{array}{c}1 \mathrm{~F} \\
129,179\end{array}$ & 1 & Del & DC & Gly & $\begin{array}{c}\text { NO, but } \\
\text { p(Gly702Val), } \\
\text { in LOVD } \\
\text { (VUS) }\end{array}$ & $\begin{array}{l}\text { Not } \\
\text { found }\end{array}$ & $\begin{array}{l}\text { Likely pathogenic, } \\
\text { PM1,PM2,PP2,PP } \\
3\end{array}$ & Strong & $\begin{array}{l}\text { YES } \\
(1 F)\end{array}$ & $\begin{array}{c}1 \mathrm{M} \\
55,168\end{array}$ \\
\hline $\begin{array}{l}107846274 \\
\text { p.(Gly743Ser }\end{array}$ & $\begin{array}{l}\text { C. } 2227 G \\
>A\end{array}$ & $\begin{array}{c}2 \mathrm{~F} \\
148,007\end{array}$ & 1 & Del & DC & Gly & $\begin{array}{c}\text { NO, but } \\
\text { p.(Gly743Asp } \\
\text { ) Plant, } 1999 \\
63 \\
\end{array}$ & $\begin{array}{l}\text { Not } \\
\text { found }\end{array}$ & $\begin{array}{c}\text { Likely pathogenic, } \\
\text { PM1,PM2, } \\
\text { PM5,PP2,PP3 }\end{array}$ & Weak & $\mathrm{NO}$ & $\begin{array}{c}2 \mathrm{~F} \\
63,637\end{array}$ \\
\hline $\begin{array}{l}107849982 \\
\text { p.(Gly752Val) }\end{array}$ & $\begin{array}{l}\text { c. } 2255 G \\
>T\end{array}$ & $\begin{array}{c}2 \mathrm{M} \\
183,227\end{array}$ & 1 & Del & DC & Gly & NO & $\begin{array}{l}\text { Not } \\
\text { found }\end{array}$ & $\begin{array}{l}\text { Likely pathogenic, } \\
\text { PM1,PM2,PP2,PP } \\
3\end{array}$ & Strong & $\begin{array}{l}\text { YES } \\
(2 \mathrm{M})\end{array}$ & \\
\hline $\begin{array}{l}107850087 \\
\text { p.(Gly787Ala) }\end{array}$ & $\begin{array}{l}\text { c. } 2360 G \\
>C\end{array}$ & $\begin{array}{c}1 \mathrm{~F} \\
183,169\end{array}$ & 1 & Del & DC & Gly & $\begin{array}{c}\text { NO, but } \\
\text { p(Gly787V } \\
\text { al), King } \\
200664\end{array}$ & $\begin{array}{l}\text { Not } \\
\text { found }\end{array}$ & $\begin{array}{c}\text { Likely pathogenic, } \\
\text { PM1,PM2, } \\
\text { PM5,PP2,PP3 }\end{array}$ & Strong & $\begin{array}{l}\text { YES } \\
(1 F)\end{array}$ & \\
\hline $\begin{array}{l}107858210 \\
\text { p.(Gly822Glu } \\
\text { ) }\end{array}$ & $\begin{array}{l}\text { C. } 2465 G \\
>A\end{array}$ & $\begin{array}{c}1 \mathrm{M} \\
182,957\end{array}$ & 1 & Del & DC & Gly & $\begin{array}{c}\text { NO, but } \\
\text { p(Gly822Ar } \\
\text { g), Cruz- } \\
\text { Robles, } \\
199965\end{array}$ & $\begin{array}{l}\text { Not } \\
\text { found }\end{array}$ & $\begin{array}{c}\text { Likely pathogenic, } \\
\text { PM1,PM2, } \\
\text { PM5,PP2,PP3 }\end{array}$ & Strong & $\begin{array}{l}\text { YES } \\
(1 \mathrm{M})\end{array}$ & \\
\hline $\begin{array}{l}107865033 \\
\text { p.(Gly893Val) }\end{array}$ & $\begin{array}{l}\text { C. } 2678 G \\
>T\end{array}$ & $\begin{array}{c}1 \mathrm{~F} \\
180,196\end{array}$ & 1 & Del & DC & Gly & \begin{tabular}{|c|} 
YES \\
Bekheirnia, \\
$2010^{66}$, and \\
p(Gly893Arg \\
) \\
Mohamma \\
d, $2014{ }^{67}$ \\
\end{tabular} & $\begin{array}{l}\text { Pathogenic } \\
\text { (no } \\
\text { assertion } \\
\text { criteria) }\end{array}$ & $\begin{array}{c}\text { Likely pathogenic, } \\
\text { PM1,PM2, } \\
\text { PM5,PP2,PP3,PP } \\
5\end{array}$ & Weak & $\begin{array}{l}\text { YES } \\
(1 F)\end{array}$ & \\
\hline p.(Gly953Val) & $\begin{array}{l}\text { c. } 2858 G \\
>T\end{array}$ & $\begin{array}{c}249 \mathrm{M}, \\
442 \mathrm{~F} \\
204,819\end{array}$ & 1 & Del & $\mathrm{P}$ & Gly & \begin{tabular}{|c|} 
YES \\
Knebelmann \\
$19966^{68}$ \\
\end{tabular} & $\begin{array}{l}\text { Conflict } \\
\text { ing } \\
\text { interpre } \\
\text { tation, } \\
\text { B, LB, } \\
\text { VUS } \\
\end{array}$ & $\begin{array}{c}\text { Benign, } \\
\text { PM1,PP2,PP3,BS } \\
\text { 1,BS2 }\end{array}$ & Benign & $\mathrm{NO}$ & $\begin{array}{c}125 \mathrm{M}, 230 \mathrm{~F}, \\
87,876\end{array}$ \\
\hline $\begin{array}{l}107866020 \\
\text { p.(Gly961Val) }\end{array}$ & $\begin{array}{l}\text { c. } 2882 \mathrm{G} \\
>\mathrm{T}\end{array}$ & $\begin{array}{l}2 \mathrm{M}, 3 \mathrm{~F} \\
182,593\end{array}$ & 0.001 & Del & $P$ & Gly & NO & $\begin{array}{l}\text { Not } \\
\text { found }\end{array}$ & $\begin{array}{c}\text { Variant of } \\
\text { Uncertain } \\
\text { Significance, } \\
\text { PM1,PP2,BP4 }\end{array}$ & Weak & $\mathrm{NO}$ & $\begin{array}{l}1 \mathrm{M}, 1 \mathrm{~F} \\
80,233\end{array}$ \\
\hline $\begin{array}{l}107866037 \\
\text { p.(Gly967Arg } \\
\text { ) }\end{array}$ & $\begin{array}{l}\text { C. } 2899 G \\
>A\end{array}$ & $\begin{array}{c}1 \mathrm{~F} \\
181,344\end{array}$ & 1 & Del & DC & Gly & NO & $\begin{array}{l}\text { Not } \\
\text { found }\end{array}$ & $\begin{array}{c}\text { Likely } \\
\text { pathogenic,PM1,P }\end{array}$ & $\begin{array}{c}\text { Moderat } \\
\text { e }\end{array}$ & $\begin{array}{l}\text { YES } \\
(1 F)\end{array}$ & \\
\hline
\end{tabular}


Copyright 2021 by ASN, Published Ahead of Print on 6/18/21, Accepted/Unedited Version

\begin{tabular}{|c|c|c|c|c|c|c|c|c|c|c|c|c|}
\hline & & & & & & & & & M2,PP2,PP3 & & & \\
\hline $\begin{array}{l}107869494 \\
p(\text { Gly1054As } \\
p)\end{array}$ & $\begin{array}{l}\text { c. } 3161 G \\
>A\end{array}$ & $\begin{array}{c}1 \mathrm{M} \\
183,204\end{array}$ & 1 & Del & DC & Gly & LOVD & $\begin{array}{l}\text { Not } \\
\text { found }\end{array}$ & $\begin{array}{l}\text { Likely pathogenic, } \\
\text { PM1,PM2,PP2,PP } \\
3\end{array}$ & $\begin{array}{c}\text { Moderat } \\
\mathrm{e}\end{array}$ & $\begin{array}{l}\text { YES } \\
(1 \mathrm{M})\end{array}$ & \\
\hline $\begin{array}{l}\text { 107869553 } \\
\text { p(Gly1074Ar } \\
\text { g) }\end{array}$ & $\begin{array}{l}\text { c. } 3220 G \\
>C\end{array}$ & $\begin{array}{c}1 \mathrm{M} \\
183,246\end{array}$ & 0.073 & Del & DC & Gly & NO & $\begin{array}{l}\text { Not } \\
\text { found }\end{array}$ & $\begin{array}{l}\text { Likely pathogenic, } \\
\text { PM1,PM2,PP2,PP } \\
3 \\
\end{array}$ & Weak & NO & \\
\hline $\begin{array}{l}107869553 \\
\text { p.(Gly1074Se } \\
\text { r) }\end{array}$ & $\begin{array}{l}\text { c. } 3220 G \\
>A\end{array}$ & $\begin{array}{c}4 \mathrm{M}, 3 \mathrm{~F} \\
204,841\end{array}$ & 0.129 & Del & DC & Gly & $\mathrm{NO}$ & $\begin{array}{l}\text { Not } \\
\text { found }\end{array}$ & $\begin{array}{c}\text { Variant of } \\
\text { Uncertain } \\
\text { Significance, } \\
\text { PM1,PP2,PP3, } \\
\text { BS2 }\end{array}$ & Weak & $\mathrm{NO}$ & $\begin{array}{l}1 \mathrm{M}, 2 \mathrm{~F}, \\
80,317\end{array}$ \\
\hline $\begin{array}{l}\text { p.(Gly1134Cy } \\
\text { s) }\end{array}$ & $\begin{array}{l}\text { c. } 3400 G \\
>T\end{array}$ & $\begin{array}{c}1 \mathrm{~F} \\
180,336\end{array}$ & 1 & Del & DC & Gly & NO & $\begin{array}{l}\text { Not } \\
\text { found }\end{array}$ & $\begin{array}{l}\text { Likely pathogenic, } \\
\text { PM1,PM2,PP2,PP } \\
3\end{array}$ & Weak & $\begin{array}{l}\text { YES } \\
(1 F)\end{array}$ & $\begin{array}{c}1 \mathrm{~F} \\
79,040\end{array}$ \\
\hline $\begin{array}{l}\text { p.(Gly1170Se } \\
\text { r) }\end{array}$ & $\begin{array}{l}\text { c. } 3508 G \\
>A\end{array}$ & $\begin{array}{c}1 \mathrm{~F} \\
174,685\end{array}$ & 1 & Del & DC & Gly & $\begin{array}{c}\text { YES Inoue, } \\
199969\end{array}$ & $\begin{array}{l}\text { Patho- } \\
\text { genic } \\
\text { (no } \\
\text { asserti } \\
\text { on } \\
\text { criteria) }\end{array}$ & $\begin{array}{c}\text { Pathogenic, } \\
\text { PS1,PM1,PM2,PM } \\
\text { 5,PP2,PP3,PP5 }\end{array}$ & Weak & $\begin{array}{l}\text { YES } \\
(1 F)\end{array}$ & $\begin{array}{c}1 \mathrm{~F} \\
76,459\end{array}$ \\
\hline $\begin{array}{l}107909824 \\
\text { p.(Gly1185Se } \\
\text { r) }\end{array}$ & $\begin{array}{l}\text { c. } 3553 G \\
>A\end{array}$ & $\begin{array}{c}1 F \\
159,615\end{array}$ & 0.99 & Del & DC & Gly & $\mathrm{NO}$ & $\begin{array}{l}\text { Not } \\
\text { found }\end{array}$ & $\begin{array}{l}\text { Pathogenic,PVS1, } \\
\text { PM1,PM2,PP2,PP } \\
3\end{array}$ & Weak & $\begin{array}{l}\text { YES } \\
(1 F)\end{array}$ & \\
\hline $\begin{array}{l}107911689 \\
\text { p.(Gly1249Ar } \\
\text { g) }\end{array}$ & $\begin{array}{l}\text { c. } 3745 G \\
>A\end{array}$ & $\begin{array}{c}1 \mathrm{~F} \\
174,771\end{array}$ & 0.99 & Del & DC & Gly & NO & $\begin{array}{l}\text { Not } \\
\text { found }\end{array}$ & $\begin{array}{l}\text { Likely pathogenic, } \\
\text { PM1,PM2,PP2,PP } \\
3\end{array}$ & Weak & $\begin{array}{l}\text { YES } \\
(1 F)\end{array}$ & \\
\hline $\begin{array}{l}107920766 \\
\text { p.(Gly1282GI } \\
u) \\
\end{array}$ & $\begin{array}{l}\text { c. } 3845 G \\
>A\end{array}$ & $\begin{array}{c}1 \mathrm{M} \\
183,125\end{array}$ & 1 & Del & DC & Gly & $\begin{array}{c}\text { NO, but } \\
p(\text { Gly1282V } \\
\text { al), in LOVD }\end{array}$ & $\begin{array}{l}\text { Not } \\
\text { found }\end{array}$ & $\begin{array}{l}\text { Likely pathogenic, } \\
\text { PM1,PM2,PP2,PP } \\
3\end{array}$ & Weak & $\begin{array}{l}\text { YES } \\
(1 \mathrm{M})\end{array}$ & \\
\hline $\begin{array}{l}107920774 \\
\text { p.(Gly1285Se } \\
\text { r) }\end{array}$ & $\begin{array}{l}\text { c. } 3853 G \\
>A\end{array}$ & $\begin{array}{c}1 \mathrm{~F} \\
183,121\end{array}$ & 0.026 & Tol & DC & Gly & $\mathrm{NO}$ & $\begin{array}{l}\text { Not } \\
\text { found }\end{array}$ & $\begin{array}{l}\text { Likely pathogenic, } \\
\text { PM1,PM2,PP2,PP } \\
3\end{array}$ & Weak & $\mathrm{NO}$ & $\begin{array}{c}1 \mathrm{~F} \\
80,288\end{array}$ \\
\hline $\begin{array}{l}107920820 \\
\text { p.(Gly1300Al } \\
\text { a) }\end{array}$ & $\begin{array}{l}\text { c. } 3899 G \\
>C\end{array}$ & $\begin{array}{c}1 \mathrm{~F} \\
182,971\end{array}$ & 0.999 & Del & DC & Gly & $\mathrm{NO}$ & $\begin{array}{l}\text { Not } \\
\text { found }\end{array}$ & $\begin{array}{l}\text { Likely pathogenic, } \\
\text { PM1,PM2,PP2,PP } \\
3 \\
\end{array}$ & Weak & $\begin{array}{l}\text { YES } \\
(1 F)\end{array}$ & \\
\hline $\begin{array}{l}107920829 \\
\text { p.(Gly1303Al } \\
\text { a) }\end{array}$ & $\begin{array}{l}\text { c. } 3908 G \\
>C\end{array}$ & $\begin{array}{c}1 \mathrm{M} \\
182,951\end{array}$ & 1 & Del & DC & Gly & NO & $\begin{array}{l}\text { Not } \\
\text { found }\end{array}$ & $\begin{array}{l}\text { Likely pathogenic, } \\
\text { PM1,PM2,PP2,PP } \\
3 \\
\end{array}$ & Weak & $\begin{array}{l}\text { YES } \\
(1 \mathrm{M})\end{array}$ & \\
\hline 107923928 & c. $3962 G$ & $1 \mathrm{~F}$ & 1 & Del & $\mathrm{DC}$ & Gly & $\mathrm{NO}$ & Not & Likely pathogenic, & Weak & YES & \\
\hline
\end{tabular}


Copyright 2021 by ASN, Published Ahead of Print on 6/18/21, Accepted/Unedited Version

\begin{tabular}{|c|c|c|c|c|c|c|c|c|c|c|c|c|}
\hline $\begin{array}{l}\text { p.(Gly1321Va } \\
\text { I) }\end{array}$ & $>T$ & 182,976 & & & & & & found & $\begin{array}{c}\text { PM1,PM2,PP2,PP } \\
3\end{array}$ & & (1F) & \\
\hline $\begin{array}{l}107923937 \\
\text { p.(Gly1324GI } \\
\text { u) }\end{array}$ & $\begin{array}{l}\text { c. } 3971 G \\
>A\end{array}$ & $\begin{array}{c}1 \mathrm{~F} \\
182,990\end{array}$ & 1 & Del & DC & Gly & NO & $\begin{array}{l}\text { Not } \\
\text { found }\end{array}$ & $\begin{array}{l}\text { Likely pathogenic, } \\
\text { PM1,PM2,PP2,PP } \\
3\end{array}$ & Weak & $\begin{array}{l}\text { YES } \\
(1 F)\end{array}$ & $\begin{array}{c}1 \mathrm{~F} \\
80,251\end{array}$ \\
\hline $\begin{array}{l}\text { p.(Gly1333Cy } \\
\text { s) }\end{array}$ & $\begin{array}{l}\text { c.3997G } \\
>T\end{array}$ & $\begin{array}{c}1 \mathrm{M}, \\
182,997\end{array}$ & 1 & Del & DC & Gly & $\begin{array}{l}\text { NO, but } \\
\text { p(Gly1333S } \\
\text { er), Plant, } \\
199963,70 \\
\end{array}$ & $\begin{array}{l}\text { Not } \\
\text { found }\end{array}$ & $\begin{array}{c}\text { Pathogenic, } \\
\text { PVS1,PM1,PM2,P } \\
\text { M5,PP2,PP3 }\end{array}$ & Weak & $\begin{array}{l}\text { YES } \\
(1 \mathrm{M})\end{array}$ & $\begin{array}{c}1 \mathrm{M} \\
80,243\end{array}$ \\
\hline $\begin{array}{l}107925082 \\
\text { p.(Gly1394Cy } \\
\text { s) }\end{array}$ & $\begin{array}{l}\text { c. } 4180 G \\
>T\end{array}$ & $\begin{array}{c}2 \mathrm{~F} \\
182,474\end{array}$ & 1 & Del & DC & Gly & NO & $\begin{array}{l}\text { Not } \\
\text { found }\end{array}$ & $\begin{array}{l}\text { Pathogenic and } \\
\text { predicted to result } \\
\text { in termination } \\
\text { codon, PP3 }\end{array}$ & $\begin{array}{c}\text { Moderat } \\
\mathrm{e}\end{array}$ & $\begin{array}{l}\text { YES } \\
(2 \mathrm{~F})\end{array}$ & \\
\hline $\begin{array}{l}107929314 \\
\text { p.(Gly1424Se } \\
\text { r) }\end{array}$ & $\begin{array}{l}\text { C. } 4270 G \\
>A\end{array}$ & $\begin{array}{c}1 \mathrm{~F} \\
182,592\end{array}$ & 1 & Del & DC & Gly & $\begin{array}{c}\text { YES } \\
\text { LOVD, and } \\
p(\text { Gly1424 } \\
\text { Glu) Zhang, } \\
2011 \\
\end{array}$ & $\begin{array}{l}\text { Not } \\
\text { found }\end{array}$ & $\begin{array}{c}\text { Likely pathogenic, } \\
\text { PM2,PM3,PM5,PP } \\
\text { 2,PP3 }\end{array}$ & Weak & $\begin{array}{l}\text { YES } \\
(1 F)\end{array}$ & \\
\hline 107802385 & $\begin{array}{l}\text { c. } 231+2 \\
T>C\end{array}$ & $\begin{array}{c}1 \mathrm{M}, \\
182,806\end{array}$ & & & & & NO & $\begin{array}{l}\text { Not } \\
\text { found }\end{array}$ & $\begin{array}{c}\text { Pathogenic, } \\
\text { PVS1,PM2,PP3 }\end{array}$ & $\begin{array}{l}\text { Exon } 3 \\
\text { skip likely, } \\
\text { likely } \\
\text { abolition of } \\
\text { donor site } \\
\text { (3/4 tools) }\end{array}$ & $\begin{array}{l}\text { YES } \\
(1 \mathrm{M})\end{array}$ & $\begin{array}{c}1 F \\
80,152\end{array}$ \\
\hline 107821614 & $\begin{array}{l}\text { c. } 780+1 \\
G>T\end{array}$ & $\begin{array}{c}1 \mathrm{~F} \\
183,138\end{array}$ & & & & & NO & $\begin{array}{l}\text { Not } \\
\text { found }\end{array}$ & $\begin{array}{c}\text { Pathogenic, } \\
\text { PVS1,PM2,PP3 }\end{array}$ & $\begin{array}{l}\text { Exon } 13 \\
\text { skip likely, } \\
\text { likely } \\
\text { abolition of } \\
\text { donor site } \\
\text { (4/4 tools) }\end{array}$ & $\begin{array}{l}\text { YES } \\
\text { (1F) }\end{array}$ & $\begin{array}{c}1 \mathrm{M}, \\
80,312\end{array}$ \\
\hline 107863487 & $\begin{array}{l}\text { c. } 1340- \\
1 \mathrm{G}>\mathrm{A}\end{array}$ & $\begin{array}{c}1 \mathrm{~F} \\
183,216\end{array}$ & & & & & NO & $\begin{array}{l}\text { Likely } \\
\text { pathog } \\
\text { enic*}\end{array}$ & $\begin{array}{c}\text { Pathogenic, } \\
\text { PVS1,PM2,PP3,P } \\
\text { P5 }\end{array}$ & $\begin{array}{c}\text { Exon21 } \\
\text { skip likely, } \\
\text { likely } \\
\text { abolition of } \\
\text { acceptor } \\
\text { site (4/4 } \\
\text { tools) } \\
\end{array}$ & $\begin{array}{l}\text { YES } \\
\text { (1F) }\end{array}$ & $\begin{array}{c}1 \mathrm{M}, \\
80,317\end{array}$ \\
\hline 107863487 & $\begin{array}{l}\text { c. } 2510- \\
2 A>G\end{array}$ & $\begin{array}{c}1 \mathrm{~F} \\
181,241\end{array}$ & & & & & NO & $\begin{array}{l}\text { Pathog } \\
\text { enic } \\
\text { (no } \\
\text { asserti } \\
\text { on } \\
\text { criteria) }\end{array}$ & $\begin{array}{c}\text { Pathogenic, } \\
\text { PVS1,PM2,PP3,P } \\
\text { P5 }\end{array}$ & $\begin{array}{c}\text { Exon } 31 \\
\text { skip likely, } \\
\text { likely } \\
\text { abolition of } \\
\text { acceptor } \\
\text { site }(3 / 4 \\
\text { tools) }\end{array}$ & $\begin{array}{l}\text { YES } \\
(1 \mathrm{~F})\end{array}$ & $\begin{array}{c}1 \mathrm{M}, \\
80,268\end{array}$ \\
\hline 107917984 & c. $3808^{+}$ & $1 \mathrm{~F}$ & & & & & NO & Not & Pathogenic, & $\begin{array}{l}\text { Exon } 43 \\
\text { skip likely, }\end{array}$ & YES & \\
\hline
\end{tabular}




\begin{tabular}{|c|c|c|c|c|c|c|c|c|c|c|c|c|}
\hline & $1 G>C$ & 169,376 & & & & & & found & $\begin{array}{c}\text { PVS1,PM2,PP3,P } \\
\text { P5 }\end{array}$ & $\begin{array}{c}\text { likely } \\
\text { abolition of } \\
\text { donor site } \\
(3 / 4)\end{array}$ & $(1 F)$ & \\
\hline 107929360 & $\begin{array}{l}\text { c. } 4315+ \\
1 G>A\end{array}$ & $\begin{array}{c}2 F \\
200,378\end{array}$ & & & & & NO & $\begin{array}{l}\text { Pathog } \\
\text { enic*}^{*}\end{array}$ & $\begin{array}{c}\text { Pathogenic, } \\
\text { PVS1,PM2,PP3,P } \\
\text { P5 }\end{array}$ & $\begin{array}{l}\text { Exon } 48 \\
\text { skip likely, } \\
\text { likely } \\
\text { abolition of } \\
\text { donor site } \\
\text { (3/4 tools) } \\
\end{array}$ & $\begin{array}{l}\text { YES } \\
(2 F)\end{array}$ & \\
\hline \multirow[t]{2}{*}{$\begin{array}{l}107898559 \\
\text { p.(Gly1083Te } \\
\text { r) }\end{array}$} & $\begin{array}{l}\text { c. } 3247 \\
3248 \\
\text { delGG }\end{array}$ & $\begin{array}{c}1 \mathrm{~F} \\
182,859\end{array}$ & & & & & NO & $\begin{array}{l}\text { Not } \\
\text { found }\end{array}$ & $\begin{array}{c}\text { Pathogenic, } \\
\text { PVS1,PM2,PP3 }\end{array}$ & $\begin{array}{l}\text { Likely } \\
\text { abolition of } \\
\text { acceptor } \\
\text { site (4/4 } \\
\text { tools) } \\
\text { interrupts } \\
\text { reading } \\
\text { frame } \\
\text { prematurel } \\
y\end{array}$ & YES & $\begin{array}{c}1 \mathrm{~F} \\
80,169\end{array}$ \\
\hline & $\begin{array}{l}34 \text { variants } \\
\text { assessed }\end{array}$ & $\begin{array}{c}88 \\
\text { individuals } \\
\text { potentially } \\
\text { affected } \\
(29 \mathrm{M}, 59 \mathrm{~F})\end{array}$ & $\begin{array}{l}47 \\
\text { variant } \\
\text { s } \\
\text { patho- } \\
\text { genic }\end{array}$ & $\begin{array}{l}47 \\
\text { variant } \\
\text { s } \\
\text { patho- } \\
\text { genic }\end{array}$ & $\begin{array}{l}52 \\
\text { variant } \\
\mathrm{s} \\
\text { patho- } \\
\text { genic }\end{array}$ & $\begin{array}{c}45 \\
\text { missense } \\
\text { variants } \\
\text { con- } \\
\text { served }\end{array}$ & $\begin{array}{l}16 \text { previous } \\
\text { reports of } \\
\text { pathogenicity }\end{array}$ & $\begin{array}{l}9 \\
\text { decisions } \\
; \\
7 \\
\text { pathogen } \\
\text { ic }\end{array}$ & $\begin{array}{l}51 \text { variants predicted } \\
\text { pathogenic in } \\
\text { Varsome }\end{array}$ & $\begin{array}{l}30 \text { variants } \\
\text { moderately } \\
\text { or strongly } \\
\text { pathogenic }\end{array}$ & $\begin{array}{l}9 \\
\text { decisions; } \\
7 \\
\text { pathogenic }\end{array}$ & $\begin{array}{l}23 \text { predicted } \\
\text { pathogenic } \\
\text { alleles } \\
(9 \mathrm{M}, 14 \mathrm{~F}) \text { in } \\
\text { mean } 73,849 \\
\text { alleles } \\
\text { examined }\end{array}$ \\
\hline
\end{tabular}

Evidence for pathogenicity is highlighted in red and evidence against pathogenicity is in blue. M male, F female. PP2 (Polyphen-2), SIFT (Sorting Intolerant from Tolerant), Mutation taster are computational tools to assess pathogenicity, and scores of $>0.80$, Del (for deleterious) or DC (disease-causing) are consistent with pathogenicity respectively.

Conservation of Gly was examined to vertebrates (in birds). ClinVar - uses P pathogenic, LP Likely pathogenic, VUS variant of uncertain significance, $B$ - benign, LB likely benign, and star system used for quality of assertion * to ****. Varsome uses ACMG/AMP grading of P, LP,VUS,LB and B and criteria of PVS, PM, PP, BS etc. While individual tools may be consistent with a pathogenic or benign classification these are not individually sufficient to assert variant pathogenicity. The tools used here have been to principally exclude variants where the evidence is not totally supportive of pathogenicity. 
Table 3: Prevalence of predicted pathogenic variants in COL4A5 in people of different ancestries in gnomAD

\begin{tabular}{|l|l|l|l|}
\hline Ethnicity & $\begin{array}{l}\text { Number of } \\
\text { individuals tested }\end{array}$ & $\begin{array}{l}\text { Number of } \\
\text { predicted } \\
\text { pathogenic } \\
\text { variants }\end{array}$ & $\begin{array}{l}\text { Prevalence of } \\
\text { predicted } \\
\text { pathogenic } \\
\text { variants in } \\
\text { COL4A5 }\end{array}$ \\
\hline African & 7 & One in 1,733 \\
\hline Ashkenazi Jewish & 4,961 & 1 & One in 4,961 \\
\hline East Asian & 9,243 & 4 & One in 2,310 \\
\hline European & 59,512 & 33 & One in 1,800 \\
\hline Finnish & 12,242 & 0 & None \\
\hline Latino & 18,105 & 6 & One in 3,018 \\
\hline South Asian & 12,948 & 4 & One in 3,237 \\
\hline Other & 3,140 & 4 & One in 785 \\
\hline Total & 136,920 & 59 & One in 2,320 \\
\hline
\end{tabular}


Table 4: Prevalence of predicted pathogenic variants in COL4A3 and COL4A4 in people of different ancestries in gnomAD

\begin{tabular}{|c|c|c|c|c|c|c|c|c|c|c|}
\hline COL4A3 & $\begin{array}{l}\text { Probably } \\
\text { pathogenic } \\
\text { variants/mea } \\
\text { n total alleles } \\
\text { examined }\end{array}$ & $\begin{array}{l}\text { Prevalenc } \\
\text { e }\end{array}$ & African & Latino & $\begin{array}{l}\text { Ashkenazi } \\
\text { m }\end{array}$ & $\begin{array}{l}\text { East } \\
\text { Asian }\end{array}$ & $\begin{array}{l}\text { Europea } \\
\text { n Finnish }\end{array}$ & $\begin{array}{l}\text { European } \\
\text { non- } \\
\text { Finnish }\end{array}$ & Other & $\begin{array}{l}\text { South } \\
\text { Asians }\end{array}$ \\
\hline $\begin{array}{l}\text { Position 1 } \\
\text { Gly } \\
\text { substitution } \\
\text { s }\end{array}$ & $380 / 234,398$ & $0.16 \%$ & $\begin{array}{l}32 / 15,28 \\
0\end{array}$ & $96 / 32,139$ & $5 / 9400$ & $\begin{array}{l}46 / 16,84 \\
2\end{array}$ & $7 / 20,376$ & $\begin{array}{l}140 / 106,28 \\
8\end{array}$ & $8 / 5739$ & $\begin{array}{l}46 / 28,33 \\
3\end{array}$ \\
\hline $\begin{array}{l}\text { Nonsense } \\
\text { variants }\end{array}$ & $33 / 245,590$ & $0.01 \%$ & $8 / 18,361$ & $4 / 32,413$ & $2 / 9,487$ & $2 / 17,393$ & $1 / 21,567$ & $12 / 111,937$ & $0 / 6,117$ & $4 / 28,315$ \\
\hline $\begin{array}{l}\text { Splicing } \\
\text { variants }\end{array}$ & $36 / 264,528$ & $0.01 \%$ & $4 / 14,588$ & $5 / 30,169$ & $0 / 8,840$ & $4 / 15,827$ & $1 / 19,207$ & $13 / 99,841$ & $2 / 5,421$ & $7 / 26,560$ \\
\hline $\begin{array}{l}\% \text { predicted } \\
\text { pathogenic } \\
\text { alleles/mean } \\
\text { total alleles }\end{array}$ & $559 / 245,889$ & $0.23 \%$ & $\begin{array}{l}47 / 15,75 \\
5 \\
(0.30 \%)\end{array}$ & $\begin{array}{l}131 / 32,71 \\
3(0.40 \%)\end{array}$ & $\begin{array}{l}7 / 9,425 \\
(0.07 \%)\end{array}$ & $\begin{array}{l}54 / 16,82 \\
9 \\
(0.32 \%)\end{array}$ & $\begin{array}{l}15 / 22,228 \\
(0.07 \%)\end{array}$ & $\begin{array}{l}213 / 111,25 \\
3 \\
(0.19 \%)\end{array}$ & $\begin{array}{l}15 / 6,16 \\
6 \\
(0.24 \%)\end{array}$ & $\begin{array}{l}75 / 28,71 \\
1 \\
(0.26 \%)\end{array}$ \\
\hline \multicolumn{11}{|l|}{ COL4A4 } \\
\hline $\begin{array}{l}\text { Position 1 } \\
\text { Gly } \\
\text { substitution } \\
\text { s }\end{array}$ & $458 / 234,586$ & $0.20 \%$ & $\begin{array}{l}44 / 15,80 \\
5\end{array}$ & $\begin{array}{l}114 / 31,85 \\
0\end{array}$ & $6 / 9,292$ & $\begin{array}{l}52 / 16,79 \\
3\end{array}$ & $9 / 20,406$ & $\begin{array}{l}174 / 106,59 \\
0\end{array}$ & $7 / 5,759$ & $\begin{array}{l}52 / 28,09 \\
0\end{array}$ \\
\hline $\begin{array}{l}\text { Frameshift } \\
\text { variants }\end{array}$ & $42 / 223,504$ & $0.02 \%$ & $5 / 14,880$ & $2 / 30,446$ & $1 / 8,878$ & $3 / 16,014$ & $1 / 19,435$ & $24 / 101,495$ & $0 / 5,482$ & $6 / 26,870$ \\
\hline Nonsense & $48 / 228,799$ & $0.02 \%$ & $2 / 15,211$ & $2 / 31,170$ & $1 / 9,098$ & $2 / 16,386$ & $0 / 19,838$ & $29 / 104,040$ & $3 / 5,609$ & $4 / 27,444$ \\
\hline
\end{tabular}


Copyright 2021 by ASN, Published Ahead of Print on 6/18/21, Accepted/Unedited Version

\begin{tabular}{|c|c|c|c|c|c|c|c|c|c|c|}
\hline variants & & & & & & & & & & \\
\hline $\begin{array}{l}\text { Splicing } \\
\text { variants }\end{array}$ & $29 / 246,884$ & $0.01 \%$ & $2 / 15,273$ & $5 / 34,351$ & $0 / 10,009$ & $3 / 17,838$ & $0 / 21,428$ & $14 / 111,706$ & $0 / 6,006$ & $5 / 30,271$ \\
\hline $\begin{array}{l}\text { \% predicted } \\
\text { pathogenic } \\
\text { alleles/mean } \\
\text { total alleles }\end{array}$ & $577 / 233,916$ & $0.25 \%$ & $\begin{array}{l}53 / 15,67 \\
5 \\
(0.34 \%)\end{array}$ & $\begin{array}{l}123 / 31,91 \\
7 \\
(0.39 \%)\end{array}$ & $\begin{array}{l}8 / 9,216 \\
(0.09 \%)\end{array}$ & $\begin{array}{l}60 / 16,75 \\
8 \\
(0.35 \%)\end{array}$ & $\begin{array}{l}10 / 20,309 \\
(0.05 \%)\end{array}$ & $\begin{array}{l}241 / 106,07 \\
3 \\
(0.23 \%)\end{array}$ & $\begin{array}{l}10 / 5,71 \\
4 \\
(0.18 \%)\end{array}$ & $\begin{array}{l}67 / 28,10 \\
4 \\
(0.24 \%)\end{array}$ \\
\hline $\begin{array}{l}\% \\
\text { individuals } \\
\text { with } \\
\text { COL4A4 } \\
\text { variant }\end{array}$ & & $0.49 \%$ & $0.68 \%$ & $0.78 \%$ & $0.18 \%$ & $0.70 \%$ & $0.10 \%$ & $0.46 \%$ & $0.36 \%$ & $0.48 \%$ \\
\hline $\begin{array}{l}\text { \% with } \\
\text { COL4A3 or } \\
\text { COL4A4 } \\
\text { variant }\end{array}$ & & $0.94 \%$ & $1.28 \%$ & $1.58 \%$ & $0.32 \%$ & $1.34 \%$ & $0.24 \%$ & $0.84 \%$ & $0.84 \%$ & $1.00 \%$ \\
\hline
\end{tabular}

These population frequencies are based on the mean total alleles examined for each variant and not on the total number of individuals examined. 


\section{Figure Legend}

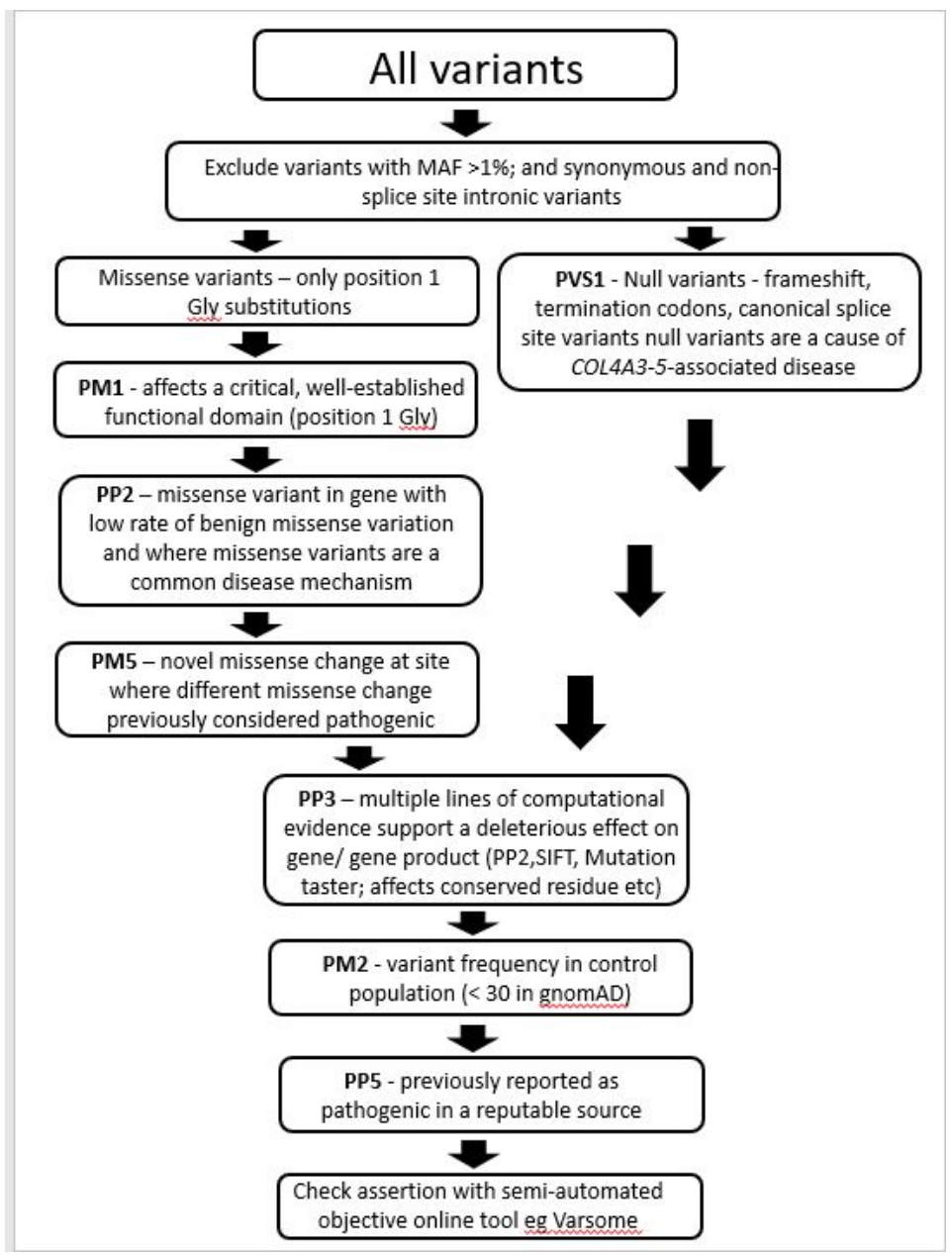

Figure 1: Filtering steps in assessment of individual COL4A3 - COL4A5 variants using ACMG/AMP criteria. PVS1 - very strong evidence of pathogenicity; PM1, PM2, PM5 - Moderate evidence of pathogenicity; PP2, PP3, PP5 - Supporting evidence of pathogenicity24. MAF - Minor Allele Frequency 
Supplementary Tables

Suppl Table 1: Applicability of ACMG/AMP criteria for COL4A3 - COL4A5 variants in population frequency databases 24

Suppl Table 2: Non-collagenous domains and interruptions in collagen IV $\square 5, \square 3$ and $\square 4$ chains $28,26,27$

Suppl Table 3: Assessment of COL4A5 variants in the 100,000 Genomes Project database and correlation with haematuria

Suppl Table 4: Assessment of COL4A3 variants in the 100,000 Genomes Project database and correlation with haematuria

Suppl Table 5: Assessment of COL4A4 variants in the 100,000 Genomes Project database and correlation with haematuria

Suppl Table 6: Predicted pathogenic variants in COL4A5 in the Exome Variant Server database

Suppl Table 7: COL4A5 predicted pathogenic variants in the TOPMed database Suppl Table 8: Mitigating features for the clinical effects of COL4A5predicted pathogenic variants resulting in position 1 Gly substitutions in gnomAD: associations with gender, location, and replacement residues Suppl Table 9: Variants in COL4A3 and COL4A4 in gnomAD with inconsistent assessments subsequently excluded from population frequency studies Suppl Table 10: Predicted pathogenic founder variants in COL4A3 and COL4A4 in different ancestries in gnomAD

Suppl Table 11: Estimated population frequencies for predicted pathogenic heterozygous, compound heterozygous, and digenic variants 


\section{Supplementary Tables}

Suppl Table 1: Applicability of ACMG/AMP criteria for COL4A3 - COL4A5 variants in population frequency databases ${ }^{24}$

Suppl Table 2: Non-collagenous domains and interruptions in collagen IV $\alpha 5, \alpha 3$ and $\alpha 4$ chains $^{28,26,27}$

Suppl Table 3: Assessment of COL4A5 variants in the 100,000 Genomes Project database and correlation with haematuria

Suppl Table 4: Assessment of COL4A3 variants in the 100,000 Genomes Project database and correlation with haematuria

Suppl Table 5: Assessment of COL4A4 variants in the 100,000 Genomes Project database and correlation with haematuria

Suppl Table 6: Predicted pathogenic variants in COL4A5 in the Exome Variant Server database

Suppl Table 7: COL4A5 predicted pathogenic variants in the TOPMed database

Suppl Table 8: Mitigating features for the clinical effects of COL4A5predicted pathogenic variants resulting in position $1 \mathrm{Gly}$ substitutions in gnomAD: associations with gender, location, and replacement residues

Suppl Table 9: Variants in COL4A3 and COL4A4 in gnomAD with inconsistent assessments subsequently excluded from population frequency studies

Suppl Table 10: Predicted pathogenic founder variants in COLAA3 and COL4A4 in different ancestries in gnomAD

Suppl Table 11: Estimated population frequencies for predicted pathogenic heterozygous, compound heterozygous, and digenic variants 


\section{Suppl Table 1: Applicability of ACMG/AMP criteria for COL4A3 - COL4A5 variants in population frequency databases ${ }^{24}$}

\begin{tabular}{|c|c|c|}
\hline Pathogenic criteria & Description & $\begin{array}{l}\text { Applicability } \\
\text { to } \\
\text { population } \\
\text { frequency } \\
\text { databases } \\
\text { for } C O L 4 A 3 \\
\text { - COL4A5 } \\
\end{array}$ \\
\hline \multicolumn{3}{|c|}{ Very strong evidence of pathogenicity (PVS) } \\
\hline PVS1 & $\begin{array}{l}\text { Null variant (nonsense, frameshift, *canonical +/- } 1 \text { or } 2 \text { splice } \\
\text { site, initiation codon, single or multi-exon deletion) }\end{array}$ & YES \\
\hline \multicolumn{3}{|c|}{ Strong evidence of pathogenicity (PS) } \\
\hline PS1 & $\begin{array}{l}\text { Same amino acid change as described previously regardless of } \\
\text { nucleotide change }\end{array}$ & YES \\
\hline PS2 & $\begin{array}{l}\text { De novo variant in a patient with the disease and no family } \\
\text { history }\end{array}$ & NO \\
\hline PS3 & $\begin{array}{l}\text { Well-established in vitro or in vivo functional studies } \\
\text { supporting a damaging effect on the gene or gene product }\end{array}$ & $\begin{array}{l}\text { No (not } \\
\text { available } \\
\text { for } \\
\text { COL4A3- } \\
\text { COL4A5) }\end{array}$ \\
\hline PS4 & $\begin{array}{l}\text { Prevalence of variant in affected individuals is significantly } \\
\text { increased compared with prevalence in controls }\end{array}$ & NO \\
\hline \multicolumn{3}{|c|}{ Moderate evidence of pathogenicity (PM) } \\
\hline PM1 & $\begin{array}{l}\text { Located in a mutational hotspot and/or critical and well- } \\
\text { established functional domain }\end{array}$ & YES** \\
\hline PM2 & Absent from controls (or low prevalence) & YES \\
\hline PM3 & For AR diseases, detected in trans with a pathogenic variant & NO \\
\hline PM4 & $\begin{array}{l}\text { Protein length changes due in in-frame deletions/insertions } \\
\text { or stop loss variants }\end{array}$ & YES \\
\hline PM5 & $\begin{array}{l}\text { Novel amino acid change in an amino acid residue where a } \\
\text { different missense change determined to be pathogenic has } \\
\text { been seen before }\end{array}$ & YES \\
\hline PM6 & $\begin{array}{l}\text { Assumed de novo, but without confirmation of paternity and } \\
\text { maternity }\end{array}$ & NO \\
\hline \multicolumn{3}{|c|}{ Supporting evidence of pathogenicity (PP) } \\
\hline PP1 & $\begin{array}{l}\text { Co-segregation with disease in multiple affected family } \\
\text { members in causative gene }\end{array}$ & NO \\
\hline PP2 & $\begin{array}{l}\text { Missense variant where these are a common mechanism of } \\
\text { disease }\end{array}$ & YES \\
\hline PP3 & $\begin{array}{l}\text { Multiple lines of computational evidence support a } \\
\text { deleterious effect (conservation, evolutionary, splicing impact } \\
\text { etc) }\end{array}$ & YES \\
\hline PP4 & $\begin{array}{l}\text { Patient's phenotype or family history is highly specific for a } \\
\text { disease with a single genetic aetiology }\end{array}$ & NO \\
\hline PP5 & $\begin{array}{l}\text { Reputable source recently reports variant as pathogenic but } \\
\text { the evidence is not available to the laboratory }\end{array}$ & YES \\
\hline
\end{tabular}




\begin{tabular}{|c|c|c|}
\hline \multicolumn{3}{|c|}{ Stand alone evidence of benign impact (BA) } \\
\hline BA1 & Allele frequency is above $5 \%$ in EVS, 1000 genomes or ExAC & NO \\
\hline \multicolumn{3}{|c|}{ Strong evidence of benign impact (BS) } \\
\hline BS1 & Allele frequency is greater than expected for disorder & YES \\
\hline BS2 & $\begin{array}{l}\text { Observed in a healthy adult individual for X-linked disorder } \\
\text { with full penetrance expected at an early age }\end{array}$ & NO \\
\hline BS3 & $\begin{array}{l}\text { Well-established in vitro or in vivo functional studies shows no } \\
\text { damaging effect on protein function or splicing }\end{array}$ & NO \\
\hline BS4 & Lack of segregation in affected members of a family & NO \\
\hline \multicolumn{3}{|c|}{ Supporting evidence of benign impact (BP) } \\
\hline BP1 & $\begin{array}{l}\text { Missense variant in a gene for which primarily truncating } \\
\text { variants are known to cause disease }\end{array}$ & NO \\
\hline BP2 & $\begin{array}{l}\text { Observed in trans with a pathogenic variant for a fully } \\
\text { penetrant dominant gene/disorder, or in cis with a pathogenic } \\
\text { variant in any inheritance pattern }\end{array}$ & NO \\
\hline BP3 & $\begin{array}{l}\text { In-frame deletions/insertions in a repetitive region without a } \\
\text { known function }\end{array}$ & YES \\
\hline BP4 & $\begin{array}{l}\text { Multiple lines of computational evidence suggest no impact } \\
\text { on gene or gene product (conservation, evolutionary, splicing } \\
\text { impact etc) }\end{array}$ & YES \\
\hline BP5 & $\begin{array}{l}\text { Variant found in a case with an alternate molecular basis for } \\
\text { disease }\end{array}$ & NO \\
\hline BP6 & $\begin{array}{l}\text { Reputable source recently reports variant as benign but the } \\
\text { evidence is not available for an independent evaluation }\end{array}$ & YES \\
\hline BP7 & $\begin{array}{l}\text { A synonymous variant for which splicing prediction algorithms } \\
\text { predict no impact to the splice consensus sequence nor the } \\
\text { creation of a new splice site AND the nucleotide is not highly } \\
\text { conserved }\end{array}$ & YES \\
\hline
\end{tabular}

PVS1, PS1, PM1 etc are explained in the Table

*A recent publication has warned against the use of PP3 for canonical splice-site variants but these are still used by Varsome ${ }^{36}$, and we did not alter Varsome assessments.

** https://www.acgs.uk.com/media/11285/uk-practice-guidelines-for-variant-classification2019-v1-0-3.pdf 
Suppl Table 2: Non-collagenous domains and interruptions in collagen IV $\alpha 5, \alpha 3$ and $\alpha 4$ chains $^{28,26,27}$

\begin{tabular}{|c|c|c|}
\hline Collagen IV $\alpha 5$ chain & Collagen IV $\alpha 3$ chain & Collagen IV $\alpha 4$ chain \\
\hline $\begin{array}{l}\text { Amino -NC domain } \\
(1-41)\end{array}$ & Amino NC (1-42) & Amino NC (1-61) \\
\hline I (160-167) & I (160-170) & I (176-183) \\
\hline II (220-223) & II (222-223) & II (235-236) \\
\hline III (243-257) & III (245-258) & III (258-269) \\
\hline IV $(282 \mid 283)^{*}$ & IV (283-287) & IV (294-295) \\
\hline V (344-355) & V (345-353) & V (359-366) \\
\hline VI (390-393) & VI (387-388) & VI (400-401) \\
\hline VII (416-419) & VII (413-414) & VII (429-432) \\
\hline VIII (442-453) & VIII (442-445) & VIII (457-462) \\
\hline IX (479-487) & IX (476-483) & IX (493-496) \\
\hline$X(549-554)$ & X (544-547) & $X(560-565)$ \\
\hline XI (595-599) & XI (587-589) & XI (605-609) \\
\hline XII (625) & XII (617-618) & XII (631-632) \\
\hline XIII (657-662) & XIII (649-655) & XIII (666-673) \\
\hline XIV (706) & XIV (698-699) & XIV (716-718) \\
\hline XV (753-756) & XV (745-749) & XV (740-741) \\
\hline XVI (818) & XVI (810-811) & XVI (763-764) \\
\hline XVII (853-856) & XVII (848-849) & XVII (828-830) \\
\hline XVIII (954-960) & XVIII (946-951) & XVIII (966-971) \\
\hline XIX (1070-1073) & XIX (1060-1064) & XIX (1014) \\
\hline XX (1189) & XX (1176-1179) & XX (1078-1081) \\
\hline XXI (1245-8) & XXI (1234-1235) & XXI (1196-1197) \\
\hline XXII (1373-78) & XXII (1263-1264) & XXII (1222-1223) \\
\hline \multirow{5}{*}{$\begin{array}{l}\text { Carboxy-NC domain } \\
(1461-1691)\end{array}$} & XXIII (1352-1357) & XXIII (1251-1257) \\
\hline & Carboxy- NC (1439-1670) & XXIV (1285-1288) \\
\hline & & XXV (1370-1379) \\
\hline & & XXVI (1404-1405) \\
\hline & & Carboxy-NC (1460-1690) \\
\hline
\end{tabular}

*This is not an interruption but rather is formed from two Gly residues adjacent to each other. 


\section{Suppl Table 3: Assessment of COL4A5 variants in the 100,000 Genomes Project database and correlation with haematuria}

\begin{tabular}{|c|c|c|c|c|c|c|c|c|c|c|c|c|}
\hline $\begin{array}{l}\text { Protein } \\
\text { consequence }\end{array}$ & Transcript & PP2 & SIFT & M T & Cons & $\begin{array}{l}\text { gnomAD } \\
\text { (Hem/ } \\
\text { Het, } \\
\text { total } \\
\text { alleles) }\end{array}$ & $\begin{array}{l}\text { Previously } \\
\text { reported; or } \\
\text { substitution } \\
\text { with other } \\
\text { residue (LOVD) }\end{array}$ & ClinVar & Varsome & $\begin{array}{l}\text { Predicted } \\
\text { pathogenic }\end{array}$ & $\begin{array}{l}\text { Number in } \\
100,000 \\
\text { Genomes } \\
\text { Project with } \\
\text { haematuria }\end{array}$ & $\begin{array}{l}\text { Number in } \\
100,000 \\
\text { Genomes } \\
\text { Project } \\
\text { without } \\
\text { haematuria }\end{array}$ \\
\hline p.(Gly93Cys) & c. $277 \mathrm{G}>\mathrm{T}$ & 1 & 0 & 1 & Gly & None & NO & & P (PM1,PM2,PM5,PP2,PP3) & YES & 1 & 0 \\
\hline p.(Gly108Glu) & $\begin{array}{c}\text { c.323G }> \\
\text { A }\end{array}$ & 1 & 0 & 1 & Gly & None & NO & & P (PM1,PM2,PM5,PP2,PP3) & YES & 1 & 0 \\
\hline p.(Gly138Cys) & c. $412 \mathrm{G}>\mathrm{T}$ & 1 & 0 & 1 & Gly & None & $\begin{array}{l}\text { NO; other - } \\
\text { LP/P }\end{array}$ & LP* & $\begin{array}{l}\text { LP (PM1,PM2,PM5,PP2, } \\
\text { PP3, PP5) }\end{array}$ & YES & 0 & 1 \\
\hline p.(Gly307Ser) & $\begin{array}{c}\text { c.919G }> \\
\text { A }\end{array}$ & 1 & 0 & 1 & Gly & None & YES- LP, other-P & & LP (PM1,PM2,PP2,PP3) & YES & 0 & 1 \\
\hline p.(Gly325Arg) & $\begin{array}{c}\text { c.973G }> \\
\text { A }\end{array}$ & 0.999 & 0 & 1 & Gly & None & YES - P & $\mathrm{P}^{*}$ & $\begin{array}{l}\text { P (PS1,PM1,PM2,PM5,PP2, } \\
\text { PP3,PP5) }\end{array}$ & YES & 1 & 0 \\
\hline p.(Gly426Arg) & $\begin{array}{c}\text { c.1276G> } \\
\text { A }\end{array}$ & 1 & 0.001 & 1 & Gly & None & YES - P & & P (PS1,PM1,PM2,PP2,PP3) & YES & 1 & 0 \\
\hline p.(Gly491Arg) & $\begin{array}{c}\text { c.1471G> } \\
\mathrm{A}\end{array}$ & 0.999 & 0.003 & 1 & Gly & None & Other - P & & LP(PM1,PM2,PM5,PP2,PP3) & YES & 0 & 1 \\
\hline p.(Gly533Arg) & $\begin{array}{c}\text { c.1597G> } \\
\mathrm{A}\end{array}$ & 1 & 0 & 1 & Gly & None & Other - P & & LP (PM1,PM2,PM5,PP2,PP3) & YES & 1 & 0 \\
\hline p.(Gly635Arg) & $\begin{array}{c}\text { c.1903G> } \\
\text { C }\end{array}$ & 1 & 0 & 1 & Gly & None & LP; other -P & & P (PM1,PM2,PM5,PP2,PP3) & YES & 1 & 0 \\
\hline GN p.(Gly624Asp) & $\begin{array}{c}\text { c.1871G> } \\
\mathrm{A}\end{array}$ & 1 & 0.021 & 1 & Gly & $\begin{array}{l}16 / \\
182,998\end{array}$ & Slajpah (2007) & $\mathrm{P}^{* *}$ & P (PM1,PP2,PP3,PP5) & YES & 0 & 1 \\
\hline p.(Gly653Val) & $\begin{array}{c}\text { c.1958G> } \\
\mathrm{T} \\
\end{array}$ & 1 & 0 & 1 & Gly & None & Other $\times 2-\mathrm{P}$ & & $\begin{array}{l}\text { LP (PM1, PM2, PM5, } \\
\text { PP2,PP3) }\end{array}$ & YES & 1 & 0 \\
\hline p.(Gly693Val) & $\begin{array}{c}\text { c. } 2078 \mathrm{G}> \\
\mathrm{T}\end{array}$ & 1 & & & Gly & None & Other $\times 2-\mathrm{LP}$ & & $\begin{array}{l}\text { LP (PM1,PM2, PM5,PP2, } \\
\text { PP3) }\end{array}$ & YES & 1 & 0 \\
\hline p.(Gly707Val) & $\begin{array}{c}\mathrm{c} .2120 \mathrm{G}> \\
\mathrm{T}\end{array}$ & 1 & 0.002 & 1 & Gly & None & NO & & LP (PM1,PM2,PP2,PP3 & YES & 1 & 0 \\
\hline p.(Gly743Ala) & $\begin{array}{c}\text { c. } 2228 \mathrm{G}> \\
\text { C }\end{array}$ & 1 & 0 & 1 & Gly & None & Other - P & & LP (PM1,PM2,PM5,PP2,PP3) & YES & 0 & 1 \\
\hline
\end{tabular}




\begin{tabular}{|c|c|c|c|c|c|c|c|c|c|c|c|c|}
\hline GN p.(Gly752Val) & $\begin{array}{c}\text { c.2255G> } \\
T\end{array}$ & 1 & 0.031 & 1 & Gly & $\begin{array}{l}2 / \\
183,277\end{array}$ & Other - P & & LP (PM1,PM2,PP2,PP3) & YES & 0 & 3 \\
\hline p.(Gly953Val) & $\begin{array}{c}c .2858 G> \\
T\end{array}$ & 1 & 0.001 & 1 & Gly & $\begin{array}{l}705 / \\
204,819\end{array}$ & $\begin{array}{l}\text { Knebelman } \\
\text { (1996) }\end{array}$ & $\begin{array}{l}\text { Conflict } \\
\text { ing* }\end{array}$ & $\begin{array}{l}\text { VUS (PM1,PP2,PP3,PP5, } \\
\text { BS1,BS2) }\end{array}$ & NO & 5 & 63 \\
\hline p.(Gly1066Ser) & $\begin{array}{c}c .3196 G> \\
A\end{array}$ & 1 & 0 & 1 & Gly & None & Other $\times 3-\mathrm{P}$ & $\mathrm{P}^{*}$ & $\begin{array}{l}\text { P (PS1, PM1,PM2, } \\
\text { PM5,PP2,PP3,PP5) }\end{array}$ & YES & 1 & 0 \\
\hline p.(Gly1388Ser) & $\begin{array}{c}c .4162 \mathrm{G}> \\
\mathrm{A}\end{array}$ & 1 & 0.005 & 1 & Gly & None & NO & & LP (PM1,PM2,PP2,PP3) & YES & 0 & 1 \\
\hline p.(Gly1421Asp) & $\begin{array}{c}c .4262 \mathrm{G}> \\
\mathrm{A}\end{array}$ & 1 & 0 & 1 & Gly & None & Other - LP & & LP (PM1,PM2,PP2,PP3) & YES & 1 & 0 \\
\hline p.(Gly1433Ser) & $\begin{array}{c}\text { c. } 4297 \mathrm{G}> \\
\mathrm{A}\end{array}$ & 1 & 0 & 1 & Gly & None & $\begin{array}{l}\text { Other }-3 \text { in } 4- \\
\text { LP }\end{array}$ & & $\begin{array}{l}\text { P (PVS1, PM1, PM2, PM5, } \\
\text { PP2, PP3) }\end{array}$ & YES & 1 & 0 \\
\hline \multicolumn{11}{|c|}{ Total number with a predicted pathogenic variant with and without haematuria respectively } & 12 & 9 \\
\hline \multicolumn{11}{|c|}{ Total number with predicted non-pathogenic variant with or without haematuria respectively } & 5 & 63 \\
\hline \multicolumn{11}{|c|}{ Total number with haematuria and without haematuria respectively } & 2221 & 37,200 \\
\hline
\end{tabular}

GnomADindicates that the variant is also found in gnomAD database. Data highlighted in blue are those with evidence against pathogenicity, and conclusion and number of individuals from 100,000 Genomes Project with this variant with or without haematuria are. PP2 (Polyphen2), SIFT (Sorting Intolerant from Tolerant), Mutation taster are computational tools to assess pathogenicity, and scores of $>0.80$, Del (for deleterious) or DC (disease-causing) are consistent with pathogenicity respectively. Conservation of Gly was examined to vertebrates (in birds). ClinVar - uses P pathogenic, LP Likely pathogenic, VUS - variant of uncertain significance, B - benign, LB likely benign, and star system used for quality of assertion * to ****. Varsome uses ACMG/AMP grading of $\mathrm{P}, \mathrm{LP}, \mathrm{VUS}, \mathrm{LB}$ and $\mathrm{B}$ and criteria of PVS, PM, PP, BS etc. The tools used here have been to principally exclude variants where the evidence is not supportive of pathogenicity.

Of especial interest was p.(Gly953Val) which was not predicted pathogenic and excluded from the population frequency. It was assessed as pathogenic in only two of the three computational tools, and had a conflicting ClinVar assessment. It was present 691 times in the gnomAD cohort almost always in people of East or South Asian ancestry. Alamut considered it Likely Pathogenic based on 2 moderate (PM1-PM6) and > 4 supporting (PP1-PP5) criteria, but Varsome assessed it as Benign (PM1, PP2, PP3, BS1, BS2). This variant has been described previously as hypomorphic ${ }^{41}$, but has also been detected on the same allele as other pathogenic COL4A5 variants ${ }^{42,43}$. A recent study with clinical data from several families concluded that it was not pathogenic ${ }^{44}$. 
Suppl Table 4: Assessment of COL4A3 variants in the 100,000 Genomes Project database and correlation with haematuria

\begin{tabular}{|c|c|c|c|c|c|c|c|c|c|c|c|c|}
\hline $\begin{array}{l}\text { Protein } \\
\text { consequence }\end{array}$ & Transcript & PP2 & SIFT & M T & Cons & $\begin{array}{l}\text { gnomA } \\
\text { D } \\
\text { (Hem/ } \\
\text { Het, } \\
\text { total } \\
\text { alleles) }\end{array}$ & $\begin{array}{l}\text { Previously } \\
\text { reported; } \\
\text { or } \\
\text { substituti } \\
\text { on with } \\
\text { other } \\
\text { residue } \\
\text { (LOVD) }\end{array}$ & ClinVar & Varsome & $\begin{array}{l}\text { Predicted } \\
\text { pathogenic }\end{array}$ & $\begin{array}{l}\text { Number in } \\
100,000 \\
\text { Genomes } \\
\text { Project with } \\
\text { haematuria }\end{array}$ & $\begin{array}{l}\text { Number in } \\
100,000 \\
\text { Genomes } \\
\text { Project } \\
\text { without } \\
\text { haematuria }\end{array}$ \\
\hline p.(Gly46Arg) & c. $136 \mathrm{G}>\mathrm{A}$ & 1 & 0 & 0.999 & Gly & $\begin{array}{l}61 / \\
280,882\end{array}$ & LB & $\mathrm{LB}^{* *}$ & LP (PM1,PM2PP2,PP3,BP6) & NO & 0 & 10 \\
\hline p.(Gly49Arg) & c. $145 G>C$ & 1 & $\begin{array}{l}0.14 \\
\text { (Tol) }\end{array}$ & & Gly & None & VUS & & VUS (PM1,PM2, PP2) & NO & 0 & 1 \\
\hline p.(Gly49Glu) & c. $146 \mathrm{G}>\mathrm{A}$ & 1 & 0.002 & 0.9999 & Gly & None & NO & & LP (PM1,PM2,PP2,PP3) & YES & 0 & 1 \\
\hline p.(Gly58Arg) & c. $172 \mathrm{G}>\mathrm{C}$ & 1 & 0 & 0.9999 & Gly & $\begin{array}{l}1 / \\
240,996\end{array}$ & Other $\times 1 \mathrm{P}$ & & LP (PM1,PM2,PM5,PP2,PP3) & YES & 0 & 1 \\
\hline p.(Gly58Asp) & c. $173 \mathrm{G}>\mathrm{A}$ & 1 & 0 & 0.9999 & Gly & None & Other $\times 1 \mathrm{P}$ & & LP (PM1,PM2,PM5,PP2,PP3) & YES & 0 & 1 \\
\hline p.(Gly58Ser) & c. $172 \mathrm{G}>\mathrm{A}$ & 1 & 0 & 0.9999 & Gly & $\begin{array}{l}6 / \\
243,384 \\
\end{array}$ & Other $\mathrm{x} 1 \mathrm{P}$ & VUS* & LP (PM1,PM2,PM5,PP2,PP3) & YES & 0 & 6 \\
\hline p.(Gly67Ter) & c.199G $>\mathrm{T}$ & & & & Gly & None & NO & & P (PVS1,PM2,PP3) & YES & 0 & 1 \\
\hline p.(Gly70Arg) & c. $208 \mathrm{G}>\mathrm{C}$ & 1 & 0.002 & 0.9999 & Gly & None & NO & & LP (PM1,PM2,PP2,PP3) & YES & 0 & 1 \\
\hline GNp.(Gly94Glu) & c. $281 \mathrm{G}>\mathrm{A}$ & 1 & 0 & 0.9999 & Gly & $\begin{array}{l}1 / \\
249,140\end{array}$ & NO & & LP (PM1,PM2,PP2,PP3) & YES & 0 & 1 \\
\hline p.(Gly94Asp) & c. $281 \mathrm{G}>\mathrm{A}$ & 1 & & & Gly & None & NO & & LP (PM1,PM2,PP2,PP3) & YES & 0 & 1 \\
\hline p.(Gly97Ser) & c. $292 \mathrm{G}>\mathrm{A}$ & 1 & & & Gly & None & NO & & LP (PM1,PM2,PP2,PP3) & YES & 0 & 1 \\
\hline p.(Gly97Cys) & c. $292 \mathrm{G}>\mathrm{T}$ & 1 & 0 & 0.9999 & Gly & None & NO & & LP (PM1,PM2,PP2,PP3) & YES & 0 & 1 \\
\hline${ }^{\mathrm{GN}} \mathrm{p} .(\mathrm{Gly} 100 \mathrm{Arg})$ & c. $298 \mathrm{G}>\mathrm{A}$ & 1 & 0 & 0.9999 & Gly & $\begin{array}{l}1 / \\
249,204 \\
\end{array}$ & NO & & LP (PM1,PM2,PP2,PP3) & YES & 0 & 1 \\
\hline p.(Gly112Ser) & c. $334 \mathrm{G}>\mathrm{A}$ & 1 & 0 & 1 & Gly & None & NO & & LP (PM1,PM2,PP2,PP3) & YES & 0 & 1 \\
\hline p.(Gly115Asp) & c. $341 \mathrm{G}>\mathrm{A}$ & & 0 & 0.9999 & Gly & None & LP & $\mathrm{P}$ & LP (PM1,PM2,PP2,PP3,PP5) & YES & 0 & 1 \\
\hline p.(Gly118Arg) & c. $352 \mathrm{G}>\mathrm{A}$ & 1 & 0 & 0.9121 & Gly & & NO & & LP (PM1,PM2,PP2,PP3) & YES & 1 & 1 \\
\hline GNp.(Gly124Glu) & c. $371 \mathrm{G}>\mathrm{A}$ & 1 & 0 & 0.9999 & Gly & $\begin{array}{l}1 / \\
249,394\end{array}$ & NO & & LP (PM1,PM2,PP2,PP3) & YES & 0 & 2 \\
\hline p.(Gly139Arg) & c. $415 G>C$ & 1 & 0 & 0.9999 & Gly & None & $P$ & VUS** & LP (PM1,PM2,PP2,PP3) & YES & 1 & 1 \\
\hline p.(Gly148Val) & c. $443 G>T$ & 1 & 0.002 & 1 & Gly & $\begin{array}{l}4 / \\
280,874\end{array}$ & LP, VUS & & LP (PM1,PM2,PP2,PP3) & YES & 1 & 1 \\
\hline p.(Gly174Val) & c. $521 G>T$ & 1 & 0 & 1 & Gly & None & Other $\times 2 \mathrm{P}$ & & LP,PM1,PM2,PM5,PP2,PP3) & YES & 1 & 0 \\
\hline
\end{tabular}




\begin{tabular}{|c|c|c|c|c|c|c|c|c|c|c|c|c|}
\hline GNp.(Gly183Asp) & c. $548 \mathrm{G}>\mathrm{A}$ & 1 & 0.001 & 1 & Gly & $\begin{array}{l}1 / \\
249,322 \\
\end{array}$ & $\begin{array}{l}\text { Other } \times 3 \\
\mathrm{P}\end{array}$ & & LP (PM1,PM2,PP2,PP3) & YES & 0 & 1 \\
\hline p.(Gly201Glu) & c. $602 \mathrm{G}>\mathrm{A}$ & 1 & 0.002 & 1 & Gly & None & NO & & LP (PM1,PM2,PP2,PP3) & YES & 0 & 2 \\
\hline p.(Gly204Val) & c. $611 \mathrm{G}>\mathrm{T}$ & 1 & 0.002 & 1 & Gly & None & NO & & LP (PM1,PM2,PP2,PP3) & YES & 0 & 1 \\
\hline p.(Gly213Arg) & c. $637 \mathrm{G}>\mathrm{A}$ & 1 & 0.001 & 0.9999 & Gly & None & NO & & P (PS1,PM1,PM2,PP2,PP3) & YES & 0 & 1 \\
\hline p.(Gly242Glu) & c. $725 \mathrm{G}>\mathrm{A}$ & 1 & 0.003 & 0.9999 & Gly & None & $\mathrm{P}$ & LP* & LP (PM1,PM2,PP2,PP3,PP5) & YES & 0 & 1 \\
\hline p.(Gly291Glu) & c. $872 \mathrm{G}>\mathrm{A}$ & 1 & 0 & 1 & Gly & None & $\mathrm{P}$ & LP & LP (PM1,PM2,PP2,PP3,PP5) & YES & 0 & 1 \\
\hline GNp.(Gly300Arg) & c. $898 \mathrm{G}>\mathrm{A}$ & 1 & 0 & 0.9999 & Gly & $\begin{array}{l}5 / \\
249,426\end{array}$ & LP, VUS & & LP (PM1,PM2,PP2,PP3) & YES & 0 & 1 \\
\hline p.(Gly372Ser) & c. $1114 \mathrm{G}>\mathrm{A}$ & 1 & 0.006 & 0.9998 & Gly & None & NO & & P (PVS1,PM1,PM2,PP2,PP3) & YES & 0 & 1 \\
\hline p.(Gly378Glu) & c. $1133 G>A$ & 1 & $\begin{array}{r}0.000 \\
2 \\
\end{array}$ & 0.9936 & Gly & None & LP & & LP (PM1,PM2,PP2,PP3) & YES & 1 & 0 \\
\hline GNp.(Gly389Asp) & c. $1166 \mathrm{G}>\mathrm{A}$ & 1 & 0.002 & 0.9999 & Gly & $\begin{array}{l}1 / \\
248,214\end{array}$ & NO & & LP (PM1,PM2,PP2,PP3) & YES & 0 & 1 \\
\hline p.(Gly407Ala) & c. $1220 G>C$ & 1 & 0.008 & 1 & Ile & None & $\begin{array}{l}\text { Other } \times 1 \\
\text { VuS }\end{array}$ & & LP (PM1,PM2,PM5,PP2,PP3) & NO & 1 & 0 \\
\hline GNp.(Gly436Ala) & c. $1307 \mathrm{G}>\mathrm{C}$ & 1 & 0.01 & 0.9999 & Gly & $\begin{array}{l}5 / \\
280,838\end{array}$ & NO & & LP (PM1,PM2,PP2,PP3) & YES & 0 & 2 \\
\hline${ }^{\mathrm{GN}} \mathrm{p} .(\mathrm{Gly} 484 \mathrm{Arg})$ & c. $1450 \mathrm{G}>\mathrm{A}$ & 1 & 0.018 & 0.9999 & Gly & $\begin{array}{l}7 / \\
249,506\end{array}$ & NO & & P (PS1,PM1,PM2,PP2,PP3) & YES & 0 & 3 \\
\hline p.(Gly487Val) & c. $1460 G>T$ & 1 & 0.012 & 0.9999 & Gly & None & $\begin{array}{l}\text { Other } \times 1 \\
\mathrm{P}\end{array}$ & & LP (PM1,PM2,PM5,PP2,PP3) & YES & 0 & 1 \\
\hline p.(Gly487Ser) & c. $1459 \mathrm{G}>\mathrm{A}$ & 1 & $\begin{array}{r}0.038 \\
(\text { Dam) } \\
\end{array}$ & $\begin{array}{r}0.7711 \\
(\mathrm{Pol}) \\
\end{array}$ & Gly & $\begin{array}{l}31 / \\
280,912 \\
\end{array}$ & $\begin{array}{l}\text { Other } \times 1 \\
\mathrm{P}\end{array}$ & & LP (PM1,PM2,PM5,PP2,PP3) & NO & 0 & 4 \\
\hline p.(Gly511Arg) & c. $1531 \mathrm{G}>\mathrm{A}$ & 1 & 0.001 & 0.9999 & Gly & None & $\begin{array}{l}\text { Other } \times 1 \\
\text { LP }\end{array}$ & & LP (PM1,PM2,PP2,PP3) & YES & 0 & 1 \\
\hline p.(Gly511Ala) & c. $1532 \mathrm{G}>\mathrm{C}$ & 1 & 0.003 & 0.9999 & Gly & None & $\begin{array}{l}\text { Other } \times 1 \\
\text { LP }\end{array}$ & & LP (PM1,PM2,PP2,PP3) & YES & 0 & 1 \\
\hline p.(Gly532Cys) & c. $1594 G>T$ & 1 & 0.002 & 1 & Gly & None & $\begin{array}{l}\text { P or VUS; } \\
\text { other } x 1 \\
\text { LP }\end{array}$ & $\mathrm{P}, \mathrm{LP} * *$ & $\begin{array}{l}\text { P (PP5, } \\
\text { PM1,PM2,PM5,Pp2,PP3) }\end{array}$ & YES & 0 & 1 \\
\hline p.(Gly560Val) & c.1679G>T & 1 & 0 & 0.9999 & Gly & None & $\mathrm{LP}$ & & LP (PM1,PM2,PP2,PP3) & YES & 1 & 0 \\
\hline p.(Gly575Arg) & c. $1723 \mathrm{G}>\mathrm{A}$ & 1 & 0 & 0.9999 & Gly & None & NO & & LP (PM1,PM2,PP2,PP3) & YES & 1 & 0 \\
\hline p.(Gly575Glu) & c. $1724 \mathrm{G}>\mathrm{A}$ & 1 & 0 & 0.9999 & Gly & None & NO & & LP (PM1,PM2,PP2,PP3) & YES & 1 & 0 \\
\hline p.(Gly575Val) & c. $1724 G>T$ & 1 & 0 & 1 & Gly & None & NO & & LP (PM1,PM2,PP2,PP3) & YES & 0 & 1 \\
\hline p.(Gly602Asp) & c. $1805 \mathrm{G}>\mathrm{A}$ & 0.795 & 0.003 & 0.9999 & Gly & None & NO & & LP (PM1,PM2, PP2,PP3) & NO & 0 & 1 \\
\hline p.(Gly611Glu) & c. $1832 \mathrm{G}>\mathrm{A}$ & 1 & 0.002 & 0.9999 & Gly & None & VUS & & LP (PM1,PM2,PM5,PP2,PP3) & YES & 0 & 1 \\
\hline GN.(Gly619Arg) & c. $1855 \mathrm{G}>\mathrm{A}$ & 1 & 0.002 & 0.9999 & Gly & $\begin{array}{l}2 / \\
246,972\end{array}$ & $\begin{array}{l}\text { LP, } P \text {, or } \\
\text { VUS }\end{array}$ & & $\mathrm{P}$ (PS1,PM1,PM2,PP2,PP3) & YES & 1 & 0 \\
\hline
\end{tabular}




\begin{tabular}{|c|c|c|c|c|c|c|c|c|c|c|c|c|}
\hline GNp.(Gly637Arg) & c. $1909 \mathrm{G}>\mathrm{A}$ & 1 & 0 & 0.9999 & Gly & $\begin{array}{l}2 / \\
246,114\end{array}$ & NO & & $\mathrm{P}(\mathrm{PS} 1, \mathrm{PM} 1, \mathrm{PM} 2, \mathrm{PP} 2, \mathrm{PP} 3)$ & YES & 0 & 1 \\
\hline $\begin{array}{l}\text { p.(Gly680AspfsTe } \\
\text { r70) }\end{array}$ & $\begin{array}{l}\text { c.2031_203 } \\
\text { 8dup }\end{array}$ & 1 & & & Gly & $\begin{array}{l}2 / \\
244,894 \\
P\end{array}$ & $\mathrm{P}$ & & P (PVS1, PM2,PP3,PP5) & YES & 0 & 2 \\
\hline p.(Gly695Glu) & c. $2084 \mathrm{G}>\mathrm{A}$ & 1 & 0 & 0.9999 & Gly & None & $\begin{array}{l}\text { Other } \times 1 \\
\text { LP, P or } \\
\text { VUS }\end{array}$ & & LP (PM1,PM2,PM5,PP2,PP3) & YES & 0 & 1 \\
\hline p.(Gly695Arg) & c. $2083 \mathrm{G}>\mathrm{A}$ & 1 & 0 & 0.9999 & Gly & $\begin{array}{l}31 / \\
278,152\end{array}$ & $\begin{array}{l}\text { LP, P or } \\
\text { VUS }\end{array}$ & $\begin{array}{l}\text { Con- } \\
\text { flicting }\end{array}$ & P (PS1, PM1,PM2,PP2,PP3) & NO & 3 & 18 \\
\hline p.(Gly700Glu) & c.2099G >A & 1 & 0.001 & 0.9999 & Gly & None & NO & & LP (PM1,PM2,PP2,PP3) & YES & 0 & 1 \\
\hline $\begin{array}{l}\text { p.(Gly721ValfsTer } \\
\text { 26) }\end{array}$ & c.2162del & 1 & 0 & 1 & Gly & $\begin{array}{l}3 / \\
243,442\end{array}$ & NO & $\mathrm{P}^{*}$ & LP (PM1,PM2,PP2,PP3) & YES & 0 & 1 \\
\hline p.(Gly730Glu) & c. $2189 \mathrm{G}>\mathrm{A}$ & 1 & 0 & 0.9999 & Gly & None & NO & VUS & LP (PM1,PM2,PP2,PP3) & YES & 0 & 1 \\
\hline p.(Gly780Glu) & c. $2339 \mathrm{G}>\mathrm{A}$ & 1 & 0 & 1 & Gly & None & LP & & LP (PM1,PM2,PP2,PP3) & YES & 0 & 1 \\
\hline p.(Gly792Glu) & c. $2375 \mathrm{G}>\mathrm{A}$ & 1 & 0 & 1 & Gly & None & $\begin{array}{l}\text { P; other } \\
\text { x1 LP }\end{array}$ & & LP (PM1,PM2,PP2,PP3) & YES & 0 & 1 \\
\hline p.(Gly801Glu) & c. $2402 \mathrm{G}>\mathrm{A}$ & 1 & 0 & 1 & Gly & None & NO & & LP (PM1,PM2,PP2,PP3) & YES & 1 & 1 \\
\hline $\begin{array}{l}\text { p.(Gly807ArgfsTer } \\
\text { 28) }\end{array}$ & c.2417dup & 1 & 0.002 & 0.9999 & Gly & $\begin{array}{l}1 / \\
172,936\end{array}$ & $\mathrm{P}$ & $\mathrm{LP} * *$ & P (PVS1,PM2,PP3,PP5) & YES & 0 & 2 \\
\hline p.(Gly812Ser) & c. $2434 \mathrm{G}>\mathrm{A}$ & 0.339 & 0.019 & $\begin{array}{l}0.9986 \\
\text { (Pol) } \\
\end{array}$ & Gly & $\begin{array}{l}10 / \\
178,112 \\
\end{array}$ & VUS & & VUS (PM1,PM2,PP2,BP4) & NO & 0 & 6 \\
\hline GNp.(Gly818Arg) & c. $2452 \mathrm{G}>\mathrm{A}$ & 1 & 0 & 1 & Gly & $\begin{array}{l}2 / \\
174,872\end{array}$ & LP, VUS & $\mathrm{P}$ & $\mathrm{P}(\mathrm{PS} 1, \mathrm{PM} 1, \mathrm{PM} 2, \mathrm{PP} 2, \mathrm{PP} 3)$ & YES & 3 & 4 \\
\hline p.(Gly824Glu) & c. $2471 \mathrm{G}>\mathrm{A}$ & 1 & 0 & 1 & Gly & None & NO & & LP (PM1,PM2,PP2,PP3) & YES & 0 & 1 \\
\hline p.(Gly856Glu) & c. $2567 \mathrm{G}>\mathrm{A}$ & 0.997 & 0 & 1 & Gly & None & $\begin{array}{l}\text { LP, P or } \\
\text { VUS }\end{array}$ & & LP (PM1,PM2,PP2,PP3) & YES & 1 & 0 \\
\hline p.(Gly874Ter) & c.2620G>T & & & 1 & Gly & None & NO & & $\mathrm{P}$ (PVS1,PM2,PP3 & YES & 0 & 1 \\
\hline $\begin{array}{l}\text { p.(Gly874AspfsTe } \\
\text { r9) }\end{array}$ & c.2621del & 1 & & & Gly & $\begin{array}{l}10 / \\
280,726\end{array}$ & NO & LP* & P (PVS1,PM2,PP3,PP5) & YES & 1 & 6 \\
\hline p.(Gly883Arg) & c. $2647 \mathrm{G}>\mathrm{A}$ & 1 & 0 & 1 & Gly & None & NO & & P (PS1,PM1,PM2,PP2,PP3) & YES & 0 & 1 \\
\hline p.(Gly895Asp) & c. $2684 \mathrm{G}>\mathrm{A}$ & 1 & 0 & 1 & Gly & None & NO & $\mathrm{LP}$ & LP (PM1,PM2,PP2,PP3,PP5) & YES & 0 & 1 \\
\hline p.(Gly943Arg) & c. $2827 \mathrm{G}>\mathrm{A}$ & 1 & 0 & 0.9999 & Gly & $\begin{array}{l}2 / \\
249,332 \\
\end{array}$ & NO & & LP (PM1,PM2,PP2,Pp3) & YES & 0 & 4 \\
\hline p.(Gly1128Ala) & c. $3383 \mathrm{G}>\mathrm{C}$ & 0.999 & 0 & 1 & Gly & None & NO & & LP (PM1,PM2,PP2,Pp3) & YES & 0 & 1 \\
\hline p.(Gly1137Ser) & c. $3409 \mathrm{G}>\mathrm{A}$ & 1 & 0 & 1 & Gly & None & $\begin{array}{l}\text { Other } \times 1 \\
\mathrm{P}\end{array}$ & & LP (PM1,PM2,PM5,PP2,PP3) & YES & 0 & 1 \\
\hline p.(Gly1152Val) & c. $3455 G>T$ & 1 & 0 & 1 & Gly & None & NO & & LP (PM1,PM2,PM5,PP2,PP3) & YES & 0 & 1 \\
\hline
\end{tabular}




\begin{tabular}{|c|c|c|c|c|c|c|c|c|c|c|c|c|}
\hline p.(Gly1158Arg) & c. $3472 \mathrm{G}>\mathrm{C}$ & 1 & 0 & 1 & Gly & $\begin{array}{l}1 / \\
249,490\end{array}$ & VUS & $\begin{array}{l}\text { Con- } \\
\text { flicting * }\end{array}$ & LP (PM1,PM2,PP2,PP3) & NO & 0 & 1 \\
\hline GNp.(Gly1167Arg) & c.3499G $>A$ & 1 & 0 & 1 & Gly & $\begin{array}{l}2 / \\
249,476 \\
\end{array}$ & $L P, P, V U S$ & $\mathrm{P}, \mathrm{LP}$ & P (PS1,PM1,PM2,PP2,PP3,PP5) & YES & 1 & 3 \\
\hline p.(Gly1198Ser) & c. $3592 \mathrm{G}>\mathrm{A}$ & 1 & 0 & 1 & Gly & None & NO & LP & $\begin{array}{l}\text { LP } \\
\text { (PM1,PM2,PM5,PP2,PP3,PP5) }\end{array}$ & YES & 1 & 0 \\
\hline${ }^{\mathrm{GN}} \mathrm{p} .(\mathrm{Gly} 1207 \mathrm{Arg})$ & c. $3619 \mathrm{G}>\mathrm{C}$ & 1 & 0.532 & 0.9981 & Gly & $\begin{array}{l}1 / \\
234,658 \\
\end{array}$ & $\begin{array}{l}\text { Other } x 1, \\
\mathrm{P}\end{array}$ & $\mathrm{P}$ & VUS (PM1PM2,PP2) & NO & 1 & 0 \\
\hline p.(Gly1231Ser) & c. $3691 \mathrm{G}>\mathrm{A}$ & 1 & 0.006 & 1 & Gly & None & $\begin{array}{l}\text { Other } \times 1, \\
\mathrm{P}\end{array}$ & VUS & LP (PM1,PM2,PP2,PP3) & YES & 0 & 3 \\
\hline${ }^{G N}$ p.(Gly1254Arg) & c. $3760 \mathrm{G}>\mathrm{C}$ & 1 & 0.002 & 1 & Gly & $\begin{array}{l}1 / \\
249,506 \\
\end{array}$ & $\mathrm{P}$ & & LP (PM1,PM2,PP2,PP3) & YES & 3 & 1 \\
\hline p.(Gly1268Glu) & c. $3803 \mathrm{G}>\mathrm{A}$ & 1 & 0 & 1 & Gly & None & NO & & LP (PM1,PM2,PP2,PP3) & YES & 0 & 1 \\
\hline${ }^{\mathrm{GN}} \mathrm{p} .(\mathrm{Gly} 1277 \mathrm{Ser})$ & c. $3829 \mathrm{G}>\mathrm{A}$ & 1 & 0 & 1 & Gly & $\begin{array}{l}102 / \\
280,750 \\
\end{array}$ & $\begin{array}{l}\text { LP, P or } \\
\text { VUS }\end{array}$ & VUS & P (PS1,PM1,PM2,PP2,PP3,PP5) & NO & 2 & 28 \\
\hline p.(Gly1280Cys) & c.3838G>T & 1 & 0 & 1 & Gly & None & NO & & LP (PM1,PM2,PP2,PP3) & YES & 1 & 1 \\
\hline p.(Gly1283Glu) & c. $3848 \mathrm{G}>\mathrm{A}$ & 1 & 0 & 1 & Gly & None & NO & & LP (PM1,PM2,PP2,PP3) & YES & 0 & 1 \\
\hline p.(Gly1298Arg) & c. $3892 \mathrm{G}>\mathrm{C}$ & 1 & 0.001 & 1 & Gly & None & NO & & LP (PM1,PM2,PP2,PP3) & YES & 1 & 0 \\
\hline p.(Gly1304Arg) & c.3910G >A & 1 & 0 & 1 & Gly & None & NO & & LP (PM1,PM2,PP2,PP3) & YES & 0 & 2 \\
\hline p.(Gly1313Glu) & c.3938G >A & 1 & 0 & 1 & Gly & None & NO & & LP (PM1,PM2,PP2,PP3) & YES & 0 & 1 \\
\hline p.(Gly1328Val) & c. $3983 \mathrm{G}>\mathrm{T}$ & 1 & 0.002 & 1 & Gly & $\begin{array}{l}8 / \\
280,792\end{array}$ & NO & & LP (PM1,PM2,PP2,PP3) & YES & 0 & 2 \\
\hline GNp.(Gly1367Arg) & c. $4099 \mathrm{G}>\mathrm{C}$ & 1 & 0 & 1 & Gly & $\begin{array}{l}1 / \\
249,320\end{array}$ & NO & & LP (PM1,PM2,PP2,PP3) & YES & 0 & 1 \\
\hline p.(Gly1376Arg) & c. $4126 \mathrm{G}>\mathrm{C}$ & 1 & 0.001 & 1 & Gly & None & NO & & LP (PM1,PM2,PP2,PP3) & YES & 0 & 1 \\
\hline GNp.(Gly1397Glu) & c. $4190 \mathrm{G}>\mathrm{A}$ & 1 & 0.002 & 1 & Gly & $\begin{array}{l}1 / \\
249,248\end{array}$ & NO & & LP (PM1,PM2,PP2,Pp3) & YES & 0 & 1 \\
\hline${ }^{\mathrm{GN} p}$.(Gly1400Glu) & c. $4199 \mathrm{G}>\mathrm{A}$ & 1 & 0 & 1 & Gly & $\begin{array}{l}2 / \\
249,256\end{array}$ & NO & & LP (PM1,PM2,PP2,PP3) & YES & 0 & 1 \\
\hline p.(Gly1418Glu) & c. $4253 \mathrm{G}>\mathrm{A}$ & 1 & 0 & 1 & Gly & None & LP & & LP (PM1,PM2,PP2,PP3) & YES & 1 & 0 \\
\hline \multicolumn{11}{|c|}{ Total number with a predicted pathogenic variant with haematuria or without respectively } & 21 & 85 \\
\hline \multicolumn{11}{|c|}{ Total number with haematuria or without haematuria respectively } & 2,221 & 37,200 \\
\hline
\end{tabular}

Variants with any evidence not consistent with pathogenicity are highlighted in blue. Otherwise notes are the same as for Suppl Table 3. 
Suppl Table 5: Assessment of COL4A4 variants in the 100,000 Genomes Project database and correlation with haematuria

\begin{tabular}{|c|c|c|c|c|c|c|c|c|c|c|c|c|}
\hline $\begin{array}{l}\text { Protein } \\
\text { consequence }\end{array}$ & Transcript & PP2 & SIFT & M T & Cons & $\begin{array}{l}\text { gnomAD } \\
\text { (Hem/ } \\
\text { Het, total } \\
\text { alleles) }\end{array}$ & $\begin{array}{l}\text { Previously } \\
\text { reported; or } \\
\text { substitution } \\
\text { with other } \\
\text { residue (LOVD) }\end{array}$ & ClinVar & Varsome & $\begin{array}{l}\text { Predicted } \\
\text { pathogenic }\end{array}$ & $\begin{array}{l}\text { Number in } \\
100,000 \\
\text { Genomes } \\
\text { Project with } \\
\text { haematuria }\end{array}$ & $\begin{array}{l}\text { Number in } \\
100,000 \\
\text { Genomes } \\
\text { Project } \\
\text { without } \\
\text { haematuria }\end{array}$ \\
\hline p.(Gly1459Val) & $\begin{array}{l}\text { c.4376G> } \\
T\end{array}$ & 0.627 & $\begin{array}{l}0.044 \\
\text { (Dam) }\end{array}$ & \begin{tabular}{|l|}
0.999 \\
4 (Pol)
\end{tabular} & Gly & $\begin{array}{l}2 / \\
274,180\end{array}$ & LP & & VUS (PM1,PM2,PP2, BP4) & NO & 0 & 1 \\
\hline${ }^{\mathrm{GN}} \mathrm{p} .(\mathrm{Gly} 1430 \mathrm{Arg})$ & $\begin{array}{l}\text { c.4288G> } \\
\mathrm{A}\end{array}$ & 1 & 0.001 & 1 & Gly & $\begin{array}{l}1 / \\
249,468\end{array}$ & NO & $\mathrm{P}^{*}$ & LP (PM1,PM2PP2,PP3,PP5) & YES & 0 & 1 \\
\hline p.(Gly1418Asp) & $\begin{array}{l}\text { c. } 4253 \mathrm{G}> \\
\mathrm{A}\end{array}$ & 1 & 0.003 & 1 & Gly & None & NO & & LP (PM1,PM2PP2,PP3) & YES & 0 & 1 \\
\hline $\begin{array}{l}\text { p.(Gly1401ProfsT } \\
\text { er31) }\end{array}$ & $\begin{array}{l}\text { c.4200_42 } \\
01 \text { del }\end{array}$ & 1 & & & Gly & None & $\mathrm{P}$ & & P (PVS1,PM2,PP3) & YES & 0 & 1 \\
\hline p.(Gly1380Asp) & $\begin{array}{l}\text { c. } 4139 \mathrm{G}> \\
\mathrm{A}\end{array}$ & 1 & 0.001 & 0.999 & Gly & None & NO & & LP (PM1,PM2,PP2,PP3) & YES & 0 & 2 \\
\hline GNp.(Gly1346Val) & $\begin{array}{l}\text { c.4037G> } \\
\mathrm{T}\end{array}$ & 1 & 0.002 & 1 & Gly & $\begin{array}{l}4 / \\
249,206\end{array}$ & NO & & LP (PM1,PM2,PP2,PP3) & YES & 0 & 2 \\
\hline GNp.(Gly1325Arg) & $\begin{array}{l}\text { c.3973G> } \\
\text { C }\end{array}$ & 1 & 0 & $\begin{array}{r}0.999 \\
9\end{array}$ & Gly & $\begin{array}{l}9 / \\
249,006\end{array}$ & NO & & $\mathrm{P}$ (PVS1,PM2,PP2,PP3) & YES & 0 & 4 \\
\hline p.(Gly1319Arg) & $\begin{array}{l}\text { c.3955G> } \\
\text { A }\end{array}$ & 1 & 0 & $\begin{array}{r}0.999 \\
9\end{array}$ & Gly & None & NO & & LP (PM1,PM2,PP2,PP3) & YES & 0 & 1 \\
\hline p.(Gly1295Asp) & $\begin{array}{l}\text { c.3884G> } \\
\mathrm{A}\end{array}$ & 1 & 0.002 & $\begin{array}{r}0.999 \\
8 \\
\end{array}$ & Gly & None & NO & & LP (PM1,PM2,PP2,PP3) & YES & 0 & 1 \\
\hline p.(Gly1292Asp) & $\begin{array}{l}\text { c.3875G> } \\
\text { A }\end{array}$ & 1 & 0 & 1 & Gly & None & $\mathrm{LP}$ & LP & LP (PM1,PM2,PP2,PP3,PP5) & YES & 1 & 0 \\
\hline p.(Gly1258Asp) & $\begin{array}{l}\text { c.3773G> } \\
\mathrm{A}\end{array}$ & 1 & 0.005 & 0.998 & Gly & None & NO & & LP (PM1,PM2,PP2,PP3) & YES & 0 & 2 \\
\hline GNp.(Gly1248Glu) & $\begin{array}{l}\text { c. } 3743 G> \\
\mathrm{A}\end{array}$ & 1 & 0.003 & $\begin{array}{r}0.999 \\
9 \\
\end{array}$ & Gly & $\begin{array}{l}17 / \\
280,842 \\
\end{array}$ & VUS & LP* & LP (PM1,PM2,PP2,PP3,PP5) & YES & 0 & 1 \\
\hline p.(Gly1230Asp) & $\begin{array}{l}\text { C.3689G> } \\
\mathrm{A}\end{array}$ & 1 & 0.003 & $\begin{array}{r}0.999 \\
9 \\
\end{array}$ & Gly & None & NO & & LP (PM1,PM2,PP2,PP3) & YES & 0 & 1 \\
\hline GNp.(Gly1201Asp) & $\begin{array}{l}\text { c. } 3602 \mathrm{G}> \\
\mathrm{A}\end{array}$ & 1 & 0 & $\begin{array}{r}0.999 \\
9 \\
\end{array}$ & Gly & $\begin{array}{l}1 / \\
249,040\end{array}$ & LP & & LP (PM1,PM2,PM5,PP2,PP3) & YES & 0 & 1 \\
\hline GNp.(Gly1178Ser) & $\begin{array}{l}\text { c.3532G> } \\
\mathrm{A}\end{array}$ & 1 & 0 & $\begin{array}{r}0.999 \\
9 \\
\end{array}$ & Gly & $\begin{array}{l}24 / \\
280,386 \\
\end{array}$ & LP, VUS & & LP (PM1,PM2,PP2,PP3) & YES & 0 & 3 \\
\hline
\end{tabular}




\begin{tabular}{|c|c|c|c|c|c|c|c|c|c|c|c|c|}
\hline GNp.(Gly1151Ala) & $\begin{array}{l}\text { c. } 3452 \mathrm{G}> \\
\text { C }\end{array}$ & 1 & 0 & 1 & Gly & $\begin{array}{l}10 / \\
249,432\end{array}$ & NO & & LP (PM1,PM2,PP2.PP3) & YES & 0 & 7 \\
\hline GNp.(Gly1136Ala) & $\begin{array}{l}\mathrm{c} .3407 \mathrm{G}> \\
\mathrm{C}\end{array}$ & 1 & 0 & $\begin{array}{r}0.999 \\
9 \\
\end{array}$ & Gly & $\begin{array}{l}3 / \\
280,616 \\
\end{array}$ & NO & & LP (PM1,PM2,PP2,PP3) & YES & 0 & 1 \\
\hline p.(Gly1124Val) & $\begin{array}{l}\mathrm{c} .3371 \mathrm{G}> \\
\mathrm{T}\end{array}$ & 1 & 0.001 & 1 & Gly & None & NO & & LP (PM1,PM2,PP2,PP3) & YES & 0 & 1 \\
\hline GNp.(Gly1103Arg) & $\begin{array}{l}\mathrm{C} .3307 \mathrm{G}> \\
\mathrm{A}\end{array}$ & 1 & 0.001 & $\begin{array}{r}0.999 \\
9 \\
\end{array}$ & Gly & $\begin{array}{l}5 / \\
249,156 \\
\end{array}$ & $P, V U S$ & LP* & LP (PM1,PM2,PP2,PP3,PP5) & YES & 0 & 2 \\
\hline p.(Gly1082Val) & $\begin{array}{l}\text { c.3245G> } \\
T\end{array}$ & 1 & 0.002 & 1 & Gly & None & NO & & LP (PM1,PM2,PP2,PP3) & YES & 0 & 1 \\
\hline $\begin{array}{l}\text { p.(Gly1069SerfsTe } \\
\text { r4) }\end{array}$ & $\begin{array}{l}\text { c.3204_32 } \\
05 \\
\text { insTCTT }\end{array}$ & 1 & & & Gly & None & NO & & $\mathrm{P}$ (PVS1,PM2,PP3) & YES & 2 & 0 \\
\hline p.(Gly1018Arg) & $\begin{array}{l}\text { c.3052G> } \\
\text { C }\end{array}$ & 1 & 0 & 1 & Gly & $\begin{array}{l}4 / \\
249,580\end{array}$ & NO & & LP (PM1,PM2,PP2,PP3) & YES & 0 & 2 \\
\hline p.(Gly1015Arg) & $\begin{array}{l}\text { c.3043G> } \\
\text { A }\end{array}$ & 1 & 0.001 & 1 & Gly & None & Other $\times 1 \mathrm{LP}$ & & $\begin{array}{l}\text { LP } \\
\text { (PM1,PM2,PM5,PP2,PP3) }\end{array}$ & YES & 0 & 1 \\
\hline p.(Gly1015Glu) & $\begin{array}{l}\text { c.3044G> } \\
\text { A }\end{array}$ & 1 & 0.002 & 1 & Gly & $\begin{array}{l}14 / \\
280,944\end{array}$ & Other x1 LP & $\begin{array}{l}\text { Con- } \\
\text { flicting* }\end{array}$ & LP ( (PM1,PM2,PP2,PP3,PP5) & NO & 1 & 8 \\
\hline GNp.(Gly996Arg) & $\begin{array}{l}\text { c. } 2986 G> \\
\mathrm{A}\end{array}$ & 1 & 0 & 1 & Gly & $\begin{array}{l}10 / \\
280,858\end{array}$ & $P$, VUS & $\mathrm{P}^{*}$ & LP (PM1,PM2,PP2,PP3,PP5) & YES & 0 & 4 \\
\hline GN p.(Gly990Asp) & $\begin{array}{l}\text { C.2969G> } \\
\mathrm{A}\end{array}$ & 1 & 0 & 1 & Gly & $\begin{array}{l}4 / \\
249,330 \\
\end{array}$ & NO & & LP (PM1,PM2,PP2,PP3) & YES & 0 & 1 \\
\hline GN p.(Gly963Glu) & $\begin{array}{l}\text { c.2888G> } \\
\mathrm{A}\end{array}$ & 0.997 & 0.272 (Tol) & 0.9999 & Gly & $\begin{array}{l}2 / \\
249,566\end{array}$ & NO & & LP (PM1.PM2,PP2,PP3) & NO & 1 & 0 \\
\hline GN p.(Gly960Arg) & $\begin{array}{l}\text { c.2878G> } \\
\mathrm{A}\end{array}$ & 1 & 0.002 & 1 & Gly & $\begin{array}{l}3 / \\
249,540\end{array}$ & NO & $\begin{array}{l}\mathrm{LP} \text { or } \mathrm{P} \\
* *\end{array}$ & $\begin{array}{l}\text { P } \\
\text { (PS1,PM1,PM2,PP2,PP3,PP5) }\end{array}$ & YES & 0 & 1 \\
\hline p.(Gly948Ala) & $\begin{array}{l}\text { c.2843G> } \\
\text { C }\end{array}$ & 1 & 0 & 0.9999 & Gly & $\begin{array}{l}3 / \\
280,952\end{array}$ & NO & & LP (PM1,PM2,PP2,PP3) & YES & 1 & 0 \\
\hline p.(Gly939Ser) & $\begin{array}{l}\mathrm{C} .2815 \mathrm{G}> \\
\mathrm{A}\end{array}$ & 1 & 0 & 0.9999 & Gly & None & NO & & LP (PM1,PM2,PP2,PP3) & YES & 0 & 1 \\
\hline p.(Gly927Glu) & $\begin{array}{l}\text { C.2780G> } \\
\mathrm{A}\end{array}$ & 1 & 0 & 1 & Gly & None & NO & & LP(PM1,PM2,PP2,PP3) & YES & 0 & 1 \\
\hline p.(Gly873Arg) & $\begin{array}{l}\text { c.2617G> } \\
\text { A }\end{array}$ & 1 & 0 & 1 & Gly & None & $P, L P$ & $\begin{array}{l}\text { Con- } \\
\text { flicting* }\end{array}$ & $\mathrm{P}$ (PM1,PM2,PP2,PP3) & NO & 1 & 1 \\
\hline GNp.(Gly864Arg) & $\begin{array}{l}\text { C.2590G> } \\
\text { A }\end{array}$ & 1 & 0 & 1 & Gly & $\begin{array}{ll}1 / \\
31,324\end{array}$ & $L P, P$ & LP* & LP (PM1,PM2,PP2,PP3,PP5) & YES & 0 & 2 \\
\hline p.(Gly861Glu) & $\begin{array}{l}\text { c. } 2582 \mathrm{G}> \\
\mathrm{A}\end{array}$ & 1 & 0.001 & 0.9999 & Gly & None & NO & & LP (PM1,PM2,PP2,PP3) & YES & 1 & 0 \\
\hline
\end{tabular}




\begin{tabular}{|c|c|c|c|c|c|c|c|c|c|c|c|c|}
\hline p.(Gly840Arg) & $\begin{array}{l}\text { C.2518G> } \\
\text { A }\end{array}$ & 1 & 0 & 1 & Gly & None & NO & & LP (PM1,PM2,PP2,PP3) & YES & 1 & 0 \\
\hline p.(Gly837Arg) & $\begin{array}{l}\text { c.2509G> } \\
\mathrm{C}\end{array}$ & 1 & 0 & 1 & Gly & None & Other $\mathrm{x} 1, \mathrm{P}$ & & LP (PM1,PM2,PM5,PP2,PP3) & YES & 0 & 2 \\
\hline p.(Gly825Ala) & $\begin{array}{l}\text { c.2474G> } \\
\text { C }\end{array}$ & 0.125 & 0.048 & 0.9981 & Gly & None & NO & & LP (PM1,PM2,PP2,PP3) & NO & 0 & 1 \\
\hline GNp.(Gly816Val) & $\begin{array}{l}\mathrm{c} .2447 \mathrm{G}> \\
\mathrm{T}\end{array}$ & 1 & 0 & 1 & Gly & $\begin{array}{l}4 / \\
249,158 \\
\end{array}$ & NO & & LP (PM1,PM2,PP2,PP3) & YES & 0 & 2 \\
\hline p.(Gly816Glu) & $\begin{array}{l}\text { c.2447G> } \\
\text { A }\end{array}$ & 1 & 0 & 1 & Gly & $\begin{array}{l}24 / \\
249,158\end{array}$ & NO & & LP (PM1,PM2,PP2,PP3) & YES & 0 & 1 \\
\hline${ }^{G N}$ p.(Gly801Ala) & $\begin{array}{l}\text { c.2402G> } \\
\text { C }\end{array}$ & 1 & 0 & 0.9999 & Gly & $\begin{array}{l}1 / \\
249,424\end{array}$ & NO & & LP (PM1,PM2,PP2,PP3) & YES & 0 & 1 \\
\hline p.(Gly801Glu) & $\begin{array}{l}\text { c.2402G> } \\
\mathrm{A}\end{array}$ & 1 & 0 & 1 & Gly & None & NO & & LP (PM1,PM2,PP2,PP3) & YES & 0 & 1 \\
\hline p.(Gly798Ser) & $\begin{array}{l}\text { c.2392G> } \\
\mathrm{A}\end{array}$ & 1 & 0.005 & 0.9999 & Gly & $\begin{array}{l}3 / \\
280,816 \\
\end{array}$ & NO & VUS* & LP (PM1,PM2,PP2,PP3) & YES & 0 & 3 \\
\hline p.(Gly783Arg) & $\begin{array}{l}\mathrm{c} .2347 \mathrm{G}> \\
\mathrm{A}\end{array}$ & 1 & 0 & 1 & Gly & None & NO & VUS* & LP (PM1,PM2,PP2,PP3) & YES & 0 & 1 \\
\hline GNp.(Gly774Arg) & $\begin{array}{l}\text { c.2320G> } \\
\text { C }\end{array}$ & 1 & 0 & 1 & Gly & $\begin{array}{l}19 / \\
280,942\end{array}$ & LP,P, VUS & $\mathrm{LP} * *$ & LP (PM1,PM2,PP2,PP3,PP5) & YES & 0 & 1 \\
\hline GNp.(Gly765Val) & $\begin{array}{l}\mathrm{c} .2294 \mathrm{G}> \\
\mathrm{T}\end{array}$ & 1 & 0.009 & 1 & Gly & $\begin{array}{l}9 / \\
249,576\end{array}$ & NO & & LP (PM1,PM2,PP2,PP3) & YES & 0 & 2 \\
\hline p.(Gly751Arg) & $\begin{array}{l}\text { c.2251G> } \\
\text { A }\end{array}$ & 1 & 0 & 1 & Gly & None & NO & & LP (PM1,PM2,PP2,PP3) & YES & 0 & 1 \\
\hline GNp.(Gly748Ser) & $\begin{array}{l}\text { c.2242G> } \\
\text { A }\end{array}$ & 0.954 & 0 & 1 & Gly & $\begin{array}{l}9 / \\
280,758\end{array}$ & NO & $\mathrm{LP} * *$ & LP (PM1,PM2,PP2,PP3,PP5) & YES & 2 & 3 \\
\hline GNp.(Gly734Ser) & $\begin{array}{l}\mathrm{C} .2200 \mathrm{G}> \\
\mathrm{A}\end{array}$ & 1 & 0.009 & 0.9999 & Gly & $\begin{array}{l}2 / \\
249,028\end{array}$ & NO & & LP (PM1,PM2,PP2,PP3) & YES & 0 & 1 \\
\hline p.(Gly698Arg) & $\begin{array}{l}\text { c.2092G> } \\
\text { A }\end{array}$ & 1 & 0 & 1 & Gly & None & NO & VUS* & LP (PM1,PM2,PP2,PP3) & YES & 1 & 0 \\
\hline p.(Gly660Asp) & $\begin{array}{l}\text { c.1979G> } \\
\text { A }\end{array}$ & 1 & 0.006 & 0.9999 & Gly & None & LP & & LP (PM1,PM2,PP2,PP3) & YES & 0 & 1 \\
\hline p.(Gly651Cys) & $\begin{array}{l}\text { c.1951G> } \\
\mathrm{T}\end{array}$ & 1 & 0 & 0.9999 & Gly & None & Other $\times 1$, LP & & LP (PM1,PM2,PP2,PP3) & YES & 0 & 1 \\
\hline p.(Gly625Glu) & $\begin{array}{l}\text { c.1874G> } \\
\mathrm{A}\end{array}$ & 1 & 0.002 & 0.9999 & Gly & None & NO & & LP (PM1,PM2,PP2,PP3) & YES & 1 & 1 \\
\hline p.(Gly596Arg) & $\begin{array}{l}\text { c.1786G> } \\
\text { A }\end{array}$ & 1 & 0 & 1 & Gly & None & NO & & LP (PM1,PM2,PP2,PP3) & YES & 0 & 1 \\
\hline $\begin{array}{l}\text { p.(Gly551TrpfsTer } \\
\text { 8) }\end{array}$ & c.1649dup & 1 & & & Gly & None & $\mathrm{LP}$ & & LP (PM1,PM2,PP2,PP3) & YES & 1 & 0 \\
\hline
\end{tabular}




\begin{tabular}{|c|c|c|c|c|c|c|c|c|c|c|c|c|}
\hline p.(Gly545Asp) & $\begin{array}{l}\text { c.1634G> } \\
\text { A }\end{array}$ & 1 & 0 & 0.9999 & Gly & None & B,VUS & & LP (PM1,PM2,PP2,PP3) & NO & 0 & 1 \\
\hline p.(Gly533Asp) & $\begin{array}{l}\text { c.1598G> } \\
\mathrm{A}\end{array}$ & 0.999 & 0 & 0.9982 & Gly & None & LP, $\mathrm{P}$ & $\mathrm{P}^{*}$ & LP (PM1,PM2,PP2,PP3,PP5) & YES & 2 & 1 \\
\hline p.(Gly524Glu) & $\begin{array}{l}\text { c.1571G> } \\
\text { A }\end{array}$ & 0.576 & 0.002 & 0.9999 & Gly & $\begin{array}{l}1 / \\
249,290\end{array}$ & LB & & LP (PM1,PM2,PP2,PP3) & NO & 0 & 1 \\
\hline $\begin{array}{l}\text { p.(Gly503Trpfs } \\
\text { Ter12) }\end{array}$ & c.1505dup & 1 & & & Gly & None & NO & & LP (PM1,PM2,PP2,PP3) & YES & 0 & 1 \\
\hline p.(Gly500Val) & $\begin{array}{l}\text { c.1499G> } \\
T\end{array}$ & 1 & 0.03 & 1 & Gly & None & NO & & LP (PM1,PM2,PP2,PP3) & YES & 0 & 1 \\
\hline p.(Gly500Ala) & $\begin{array}{l}\text { c.1499G> } \\
\text { C }\end{array}$ & 1 & 0.186 Tol) & 0.9998 & Gly & None & NO & & LP (PM1,PM2,PP2,PP3) & NO & 0 & 1 \\
\hline p.(Gly479Arg) & $\begin{array}{l}\text { c.1435G> } \\
\text { C }\end{array}$ & 0 & 0.466 (Tol) & $\begin{array}{c}0.9975 \\
\text { (Pol) }\end{array}$ & Gly & $\begin{array}{l}106 / \\
280,814\end{array}$ & & $\begin{array}{l}\text { Con- } \\
\text { flicting* }\end{array}$ & VUS (PM1,PM2,PP2, BP4) & NO & 2 & 8 \\
\hline p.(Gly475Ala) & $\begin{array}{l}\text { c.1424G> } \\
\text { C }\end{array}$ & 0.991 & 1 & 0.9999 & Gly & None & NO & LP & LP (PM1,PM2,PM5,PP2,PP3) & YES & 0 & 1 \\
\hline p.(Gly451Asp) & $\begin{array}{l}\text { C.1352G> } \\
\text { A }\end{array}$ & 1 & 0.002 & 0.9999 & Gly & None & Other $x 1$ VUS & & LP (PM1,PM2,PP2,PP3) & YES & 1 & 0 \\
\hline p.(Gly442Ser) & $\begin{array}{l}\text { c.1324G> } \\
\text { A }\end{array}$ & 0.997 & 0.06 & 0.9999 & Gly & None & NO & & LP (PM1,PM2,PP2,PP3) & YES & 0 & 1 \\
\hline GNp.(Gly426Arg) & $\begin{array}{l}\text { C.1276G> } \\
\text { A }\end{array}$ & 1 & 0.001 & 0.9999 & Gly & $\begin{array}{l}4 / \\
248,782\end{array}$ & NO & & LP (PM1,PM2,PP2,PP3) & YES & 0 & 3 \\
\hline GNp.(Gly402Asp) & $\begin{array}{l}\text { c.1205G> } \\
\text { A }\end{array}$ & 1 & 0.003 & 0.9999 & Gly & $\begin{array}{l}5 / \\
247,742\end{array}$ & NO & & LP (PM1,PM2,PP2,PP3) & YES & 0 & 3 \\
\hline p.(Gly382Ala) & $\begin{array}{l}\text { c.1145G> } \\
\text { C }\end{array}$ & 0.999 & 0 & 0.9999 & Gly & None & NO & & LP (PM1,PM2,PP2,PP3) & YES & 1 & 0 \\
\hline p.(Gly379Arg) & $\begin{array}{l}\text { c.1135G> } \\
\text { A }\end{array}$ & 1 & 0 & 0.9999 & Gly & None & NO & & LP (PM1,PM2,PP2,PP3) & YES & 1 & 0 \\
\hline p.(Gly379Ala) & $\begin{array}{l}\text { c.1136G> } \\
\mathrm{C}\end{array}$ & 0.999 & 0 & 0.9999 & Gly & None & NO & & LP (PM1,PM2,PP2,PP3) & YES & 0 & 1 \\
\hline GNp.(Gly373Glu) & $\begin{array}{l}\text { c.1118G> } \\
\text { A }\end{array}$ & 1 & 0 & 0.9999 & Gly & $\begin{array}{l}2 / \\
248,186\end{array}$ & LP, VUS & $\mathrm{P}^{*}$ & LP (PM1,PM2,PP2,PP3,PP5) & YES & 2 & 2 \\
\hline p.(Gly335Val) & $\begin{array}{l}\text { c.1004G> } \\
\mathrm{T}\end{array}$ & 1 & 0 & 1 & Gly & None & NO & & LP (PM1,PM2,PP2,PP3) & YES & 1 & 0 \\
\hline p.(Gly308Glu) & c. $923 \mathrm{G}>\mathrm{A}$ & 1 & 0 & 0.9999 & Gly & None & NO & & LP (PM1,PM2,PP2,PP3) & YES & 0 & 2 \\
\hline p.(Gly291Glu) & c. $872 \mathrm{G}>\mathrm{A}$ & 1 & 0.004 & 1 & Gly & None & NO & & LP (PM1,PM2,PP2,PP3) & YES & 0 & 1 \\
\hline p.(Gly252Ser) & c. $754 \mathrm{G}>\mathrm{A}$ & 1 & 0.009 & 0.9999 & Gly & None & Other $\times 1$ VUS & & LP (PM1,PM2,PM5,PP2,PP3) & YES & 0 & 1 \\
\hline $\mathrm{GN}_{\mathrm{p}}$.(Gly190Arg) & c. $568 \mathrm{G}>\mathrm{C}$ & 1 & 0 & 1 & Gly & $\begin{array}{l}1 / \\
249,424 \\
\end{array}$ & NO & & LP (PM1,PM2,PP2,PP3) & YES & 1 & 0 \\
\hline p.(Gly164Val) & c. $491 \mathrm{G}>\mathrm{T}$ & 1 & 0 & 1 & Gly & None & NO & & LP (PM1,PM2,PP2,PP3) & YES & 0 & 1 \\
\hline
\end{tabular}




\begin{tabular}{|c|c|c|c|c|c|c|c|c|c|c|c|c|}
\hline p.(Gly161Val) & c. $482 \mathrm{G}>\mathrm{T}$ & 1 & 0 & 1 & Gly & $\begin{array}{l}4 / \\
249,086\end{array}$ & $\begin{array}{l}\text { VUS; Other } \times 2 \\
\text { LP, VUS }\end{array}$ & LP* & $\begin{array}{l}\text { LP } \\
\text { (PM1,PM2,PM5,PP2,PP3,PP5) }\end{array}$ & YES & 0 & 2 \\
\hline GNp.(Gly161Arg) & c. $481 \mathrm{G}>\mathrm{C}$ & 1 & 0 & 1 & Gly & $\begin{array}{l}3 / \\
280,524 \\
\end{array}$ & NO & LP* & $\begin{array}{l}\text { LP } \\
\text { (PM1,PM2,PM5,PP2,PP3,PP5) }\end{array}$ & YES & 0 & 2 \\
\hline p.(Gly143Asp) & c. $428 \mathrm{G}>\mathrm{A}$ & 1 & 0 & 1 & Gly & None & NO & & $\mathrm{LP}(\mathrm{PM} 1, \mathrm{PM} 2, \mathrm{PP} 2, \mathrm{PP} 3)$ & YES & 0 & 1 \\
\hline GNp.(Gly137Ser) & c. $409 \mathrm{G}>\mathrm{A}$ & 1 & 0 & 1 & Gly & $\begin{array}{l}1 / \\
248,804\end{array}$ & LP, VUS & & LP (PM1,PM2,PP2,PP3) & YES & 0 & 1 \\
\hline p.(Gly122Ser) & c. $364 \mathrm{G}>\mathrm{A}$ & 1 & 0 & 0.9999 & Gly & None & NO & & LP (PM1,PM2,PP2,PP3) & YES & 0 & 1 \\
\hline p.(Gly113Asp) & c. $338 \mathrm{G}>\mathrm{A}$ & 1 & 0 & 0.9999 & Gly & $\begin{array}{l}14 / \\
257,452\end{array}$ & NO & VUS & LP (PM1,PM2,PP2,PP3) & YES & 0 & 2 \\
\hline GNp.(Gly98Ser) & c. $292 \mathrm{G}>\mathrm{A}$ & 1 & 0 & 0.9999 & Gly & $\begin{array}{l}1 / \\
247,318\end{array}$ & NO & & LP (PM1,PM2,PP2,PP3) & YES & 0 & 3 \\
\hline $\begin{array}{l}\text { p.(Gly92Glufs } \\
\text { Ter2) }\end{array}$ & c.275del & 1 & 0 & 1 & Gly & None & NO & & LP (PM1,PM2,PP2,PP3) & YES & 0 & 1 \\
\hline \multicolumn{11}{|c|}{ Total number with a predicted pathogenic variant with and without haematuria respectively } & 19 & 95 \\
\hline Total number & haematur & $\mathrm{nd} n$ & 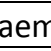 & aturia re & spect & & & & & & 2,221 & 37,200 \\
\hline
\end{tabular}

Variants with any evidence not consistent with pathogenicity are highlighted in blue. Otherwise notes are the same as for Suppl Table 3. 
Suppl Table 6: Predicted pathogenic variants in COL4A5 in the Exome Variant Server database

\begin{tabular}{|c|c|c|c|c|c|c|c|c|c|c|c|}
\hline $\begin{array}{l}\text { Protein } \\
\text { consequence }\end{array}$ & Transcript & $\begin{array}{l}\text { Affected } \\
\text { individuals/ } \\
\text { total allele } \\
\text { count }\end{array}$ & PP2 & SIFT & $\begin{array}{l}\text { Mutation } \\
\text { taster }\end{array}$ & Conserved & $\begin{array}{l}\text { Previously } \\
\text { reported; or } \\
\text { substitution with } \\
\text { other residue } \\
\text { (LOVD) }\end{array}$ & ClinVar & Varsome & Alamut & $\begin{array}{l}\text { Predicted } \\
\text { pathogenic }\end{array}$ \\
\hline${ }^{\mathrm{GN}}$ p.(Gly42Ser) & c. $124 \mathrm{G}>\mathrm{A}$ & $\begin{array}{l}1 \mathrm{M} / \\
10,563\end{array}$ & Benign (0.013) & $\begin{array}{l}\text { Damaging } \\
(0.047)\end{array}$ & $\begin{array}{l}\text { Disease } \\
\text { causing } \\
(0.99)\end{array}$ & Gly & NO & & $\begin{array}{l}\text { Likely } \\
\text { pathogenic } \\
\text { (PM1, PM2, } \\
\text { PP2, PP3) }\end{array}$ & Weak & NO \\
\hline GNp.(Gly624Asp) & c. $1871 \mathrm{G}>\mathrm{A}$ & $\begin{array}{l}1 F / \\
10,563\end{array}$ & $\begin{array}{l}\text { Prob } \\
\text { damaging } \\
(0.999)\end{array}$ & $\begin{array}{l}\text { Damaging } \\
(0.119)\end{array}$ & $\begin{array}{l}\text { Disease } \\
\text { causing } \\
(1.00)\end{array}$ & Gly & Slajpah, 2007 & $\begin{array}{l}\text { Pathogenic } \\
* *\end{array}$ & $\begin{array}{l}\text { Pathogenic } \\
\text { (PM1, PP2, } \\
\text { PP3, PP5) }\end{array}$ & Strong & YES \\
\hline p.(Gly905Ser) & c. $2713 \mathrm{G}>\mathrm{A}$ & $1 F / 10,563$ & $\begin{array}{l}\text { Probably } \\
\text { damaging } \\
1.00\end{array}$ & $\begin{array}{l}\text { Damaging } \\
(0.00)\end{array}$ & $\begin{array}{l}\text { Disease } \\
\text { causing } \\
(1.00)\end{array}$ & Gly & NO & & $\begin{array}{l}\text { Likely } \\
\text { pathogenic } \\
\text { (PM1, PM2, } \\
\text { PP2, PP3) }\end{array}$ & Strong & YES \\
\hline GNp.(Gly953Val) & c. $2858 \mathrm{G}>\mathrm{T}$ & $\begin{array}{l}2 \mathrm{M} / \\
10,563\end{array}$ & $\begin{array}{l}\text { Probably } \\
\text { damaging } \\
1.00\end{array}$ & $\begin{array}{l}\text { Damaging } \\
(0.002)\end{array}$ & $\begin{array}{l}\text { Disease } \\
\text { causing } \\
(1.00)\end{array}$ & Gly & $\begin{array}{l}\text { Knebelmann, } \\
1996\end{array}$ & $\begin{array}{l}\text { Conflicting } \\
\text { B, LB, VUS }\end{array}$ & $\begin{array}{l}\text { Benign } \\
\text { (PM1, PP2, } \\
\text { PP3,PP5, } \\
\text { BS1, BS2) } \\
\end{array}$ & Weak & NO \\
\hline GNp.(Gly1074Arg) & c. $3220 \mathrm{G}>\mathrm{C}$ & $\begin{array}{l}1 \mathrm{M} / \\
10,563\end{array}$ & Benign (0.09) & $\begin{array}{l}\text { Damaging } \\
\text { (0) }\end{array}$ & $\begin{array}{l}\text { Disease } \\
\text { causing } \\
(0.999)\end{array}$ & Gly & NO & & $\begin{array}{l}\text { Likely } \\
\text { pathogenic } \\
\text { (PM1, } \\
\text { PM2,PP2,P } \\
\text { P3) }\end{array}$ & Weak & NO \\
\hline${ }^{G N} p .(G l y 1244 S e r)$ & c. $3730 \mathrm{G}>\mathrm{A}$ & $\begin{array}{l}1 F / \\
10,563\end{array}$ & Benign (0.315) & $\begin{array}{l}\text { Damaging } \\
(0.06)\end{array}$ & $\begin{array}{l}\text { Disease } \\
\text { causing } \\
(1.00)\end{array}$ & Gly & NO & & $\begin{array}{l}\text { Likely } \\
\text { pathogenic } \\
\text { (PM1, PM2, } \\
\text { PM5, } \\
\text { PP2,PP3) }\end{array}$ & & NO \\
\hline${ }^{\mathrm{GN}} \mathrm{p}$. (Gly1424Ser) & c. $4270 \mathrm{G}>\mathrm{A}$ & $\begin{array}{l}1 \mathrm{M} / \\
10,563\end{array}$ & $\begin{array}{l}\text { Probably } \\
\text { damaging } \\
1.00\end{array}$ & $\begin{array}{l}\text { Damaging } \\
\text { (0) }\end{array}$ & $\begin{array}{l}\text { Disease } \\
\text { causing } \\
(1.00)\end{array}$ & Gly & $\begin{array}{l}\text { LOVD, } \\
\text { p.(Gly1424Glu), } \\
\text { Zhang, } 2011\end{array}$ & & $\begin{array}{l}\text { Likely } \\
\text { pathogenic, } \\
\text { PM2,PM3,P } \\
\text { M5,PP2,PP } \\
3\end{array}$ & Weak & YES \\
\hline
\end{tabular}

Evidence against pathogenicity is highlighted in blue. Three variants were predicted to be pathogenic in the cohort of 7042 individuals; $p$.(Gly624Asp), p.(Gly905Ser); and p.(Gly1424Ser). This cohort had no frameshift, termination codons or splice site variants. M male; F female. PP2 Polyphen-2, SIFT,

Mutation taster computational tools assess pathogenicity. Conserved residue in vertebrates. ClinVar - star system used for quality of assertion $*$ to $* * * *$.

P Pathogenic, LP, Likely pathogenic, B - benign, LB likely benign, VUS - variant of uncertain significance. PVS, PM, PP, BS are ACMG/AMP criteria referring to criteria for $\mathrm{P}$ pathogenicity; and $\mathrm{B}$ benign nature; $\mathrm{p}$.(Leu691Phefs* 7$)^{1}$ where ${ }^{1}$ read depth $=14$ was excluded where all other read depths were $>30$. 
Suppl Table 7: Predicted pathogenic variants in COL4A5 in the TOPMed database

\begin{tabular}{|c|c|c|c|c|c|c|c|c|c|c|}
\hline $\begin{array}{l}\text { Protein } \\
\text { consequence }\end{array}$ & Transcript & $\begin{array}{l}\text { Affected } \\
\text { individuals/ } \\
\text { total allele } \\
\text { count = } \\
125,568\end{array}$ & PP2 & SIFT & $\begin{array}{l}\text { Mutation } \\
\text { taster }\end{array}$ & Conserved & $\begin{array}{l}\text { Previous } \\
\text { reports of } \\
\text { variant, or } \\
\text { other variants } \\
\text { at this } \\
\text { location } \\
\text { (LOVD) }\end{array}$ & ClinVar & Varsome & $\begin{array}{l}\text { Predicted } \\
\text { pathogenicity }\end{array}$ \\
\hline \multirow[t]{2}{*}{ In gnomAD } & c. $231+2 T>C$ & $1 \mathrm{~F}$ & & & $\begin{array}{l}\text { Disease- } \\
\text { causing }\end{array}$ & & & Not found & $\begin{array}{l}\text { Pathogenic (PVS1, } \\
\text { PM2,PP3) }\end{array}$ & YES \\
\hline & $\begin{array}{l}\text { c.1024_103 } \\
\text { 2+22delCCT } \\
\text { GGACTTGT } \\
\text { AAGTTTTTT } \\
\text { TTTTTTTAG } \\
\text { TCT }\end{array}$ & $1 \mathrm{~F}$ & & & $\begin{array}{l}\text { Disease- } \\
\text { causing }\end{array}$ & & & Not found & $\begin{array}{l}\text { Pathogenic } \\
\text { (PVS1,PM2,PP3) }\end{array}$ & YES \\
\hline p.(Gly57Arg) & c. $169 \mathrm{G}>\mathrm{C}$ & $1 \mathrm{~F}$ & 1.00 & $\begin{array}{l}\text { Damaging } \\
\text { (0) }\end{array}$ & $\begin{array}{l}\text { Disease- } \\
\text { causing }\end{array}$ & Gly & NO & Not found & $\begin{array}{l}\text { Likely pathogenic } \\
\text { (PM1,PM2,PP2,PP3) }\end{array}$ & YES \\
\hline p.(Gly111Ser) & c. $331 \mathrm{G}>\mathrm{A}$ & $1 \mathrm{~F}$ & 1.00 & $\begin{array}{l}\text { Damaging } \\
(0)\end{array}$ & $\begin{array}{l}\text { Disease- } \\
\text { causing }\end{array}$ & Gly & NO & Not found & $\begin{array}{l}\text { Likely pathogenic } \\
\text { (PM1,PM2,PP2,PP3) }\end{array}$ & YES \\
\hline $\mathrm{GN}_{\mathrm{p} . \text { (Gly279Arg }}$ & c. $835 \mathrm{G}>\mathrm{C}$ & $2 \mathrm{~F}$ & 0.999 & $\begin{array}{l}\text { Damaging } \\
(0.003)\end{array}$ & $\begin{array}{l}\text { Disease- } \\
\text { causing }\end{array}$ & Gly & NO & Not found & $\begin{array}{l}\text { Likely pathogenic } \\
\text { (PM1,PM2,PP2,PP3) }\end{array}$ & YES \\
\hline p.(Gly282Ser) & c. $844 \mathrm{G}>\mathrm{A}$ & $1 \mathrm{~F}$ & 1.00 & $\begin{array}{l}\text { Damaging } \\
(0)\end{array}$ & $\begin{array}{l}\text { Disease- } \\
\text { causing }\end{array}$ & Gly & NO & Not found & $\begin{array}{l}\text { Likely pathogenic } \\
\text { (PM1,PM2,PP2,PP3) }\end{array}$ & YES \\
\hline $\mathrm{GN}_{\mathrm{p} . \text { (Gly512Glu) }}$ & c. $1535 \mathrm{G}>\mathrm{A}$ & $2 \mathrm{~F}$ & 1.00 & Damaging & $\begin{array}{l}\text { Disease- } \\
\text { causing }\end{array}$ & Gly & NO & Not found & $\begin{array}{l}\text { Likely pathogenic } \\
\text { (PM1,PM2,PP2,PP3) }\end{array}$ & YES \\
\hline $\mathrm{GN}_{\text {p.(Gly624Asp) }}$ & c. $2073 \mathrm{G}>\mathrm{A}$ & $2 \mathrm{M}, 7 \mathrm{~F}$ & 0.99 & $\begin{array}{l}\text { Damaging } \\
\text { (0) }\end{array}$ & $\begin{array}{l}\text { Disease- } \\
\text { causing }\end{array}$ & Gly & YES & Pathogenic ** & $\begin{array}{l}\text { Pathogenic (PM1, } \\
\text { PP2, PP3, PP5) }\end{array}$ & YES \\
\hline $\mathrm{GN}_{\text {p.(Gly } 752 \text { Val) }}$ & c. $2255 \mathrm{G}>\mathrm{T}$ & $1 \mathrm{~F}$ & 1.00 & $\begin{array}{l}\text { Damaging } \\
(0.031)\end{array}$ & $\begin{array}{l}\text { Disease- } \\
\text { causing }\end{array}$ & Gly & NO & Not found & $\begin{array}{l}\text { Likely pathogenic } \\
\text { (PM1,PM2,PP2,PP3) }\end{array}$ & YES \\
\hline p.(Gly769Glu) & c. $2306 \mathrm{G}>\mathrm{A}$ & $1 \mathrm{~F}$ & 1.00 & $\begin{array}{l}\text { Damaging } \\
\text { (0) }\end{array}$ & $\begin{array}{l}\text { Disease- } \\
\text { causing }\end{array}$ & Gly & NO & Not found & $\begin{array}{l}\text { Likely pathogenic } \\
\text { (PM1,PM2,PM5,PP2 } \\
\text {,PP3) }\end{array}$ & YES \\
\hline p.(Gly817Arg) & c. $2449 \mathrm{G}>\mathrm{C}$ & $2 \mathrm{M}, 4 \mathrm{~F}$ & 1.00 & $\begin{array}{l}\text { Damaging } \\
(0.001)\end{array}$ & $\begin{array}{l}\text { Disease- } \\
\text { causing }\end{array}$ & Gly & NO & Not found & $\begin{array}{l}\text { Likely pathogenic } \\
\text { (PM1,PM2,PP2,PP3) }\end{array}$ & YES \\
\hline p.(Gly905Ser) & c. $2713 \mathrm{G}>\mathrm{A}$ & $2 \mathrm{~F}$ & 1.00 & $\begin{array}{l}\text { Damaging } \\
(0.001)\end{array}$ & $\begin{array}{l}\text { Disease- } \\
\text { causing }\end{array}$ & Gly & NO & Not found & $\begin{array}{l}\text { Likely pathogenic } \\
\text { (PM1,PM2,PM5,PP2 } \\
\text {,PP3) }\end{array}$ & YES \\
\hline
\end{tabular}




\begin{tabular}{|c|c|c|c|c|c|c|c|c|c|c|}
\hline p.(Gly973Ser) & c. $2917 \mathrm{G}>\mathrm{A}$ & $1 \mathrm{~F}$ & 1.00 & $\begin{array}{l}\text { Damaging } \\
\text { (0) }\end{array}$ & $\begin{array}{l}\text { Disease- } \\
\text { causing }\end{array}$ & Gly & NO & Not found & $\begin{array}{l}\text { Pathogenic (PVS1, } \\
\text { PM1,PM2,PM5, } \\
\text { PP2,PP3) }\end{array}$ & YES \\
\hline p.(Gly1069Val) & c. $3206 \mathrm{G}>\mathrm{T}$ & $1 \mathrm{~F}$ & 1.00 & $\begin{array}{l}\text { Damaging } \\
\text { (0) }\end{array}$ & $\begin{array}{l}\text { Disease- } \\
\text { causing }\end{array}$ & Gly & VUS & Conflicting * & $\begin{array}{l}\text { Likely pathogenic } \\
\text { (PM1,PM2,PP2,PP3) }\end{array}$ & NO \\
\hline p.(Gly1113Ala) & c. $3338 \mathrm{G}>\mathrm{C}$ & $1 \mathrm{~F}$ & 1.00 & $\begin{array}{l}\text { Damaging } \\
(0.01)\end{array}$ & $\begin{array}{l}\text { Disease- } \\
\text { causing }\end{array}$ & Gly & NO & Not found & $\begin{array}{l}\text { Likely pathogenic } \\
\text { (PM1,PM2,PP2,PP3) }\end{array}$ & YES \\
\hline p.(Gly1119Ala) & c. $3356 \mathrm{G}>\mathrm{C}$ & $1 \mathrm{~F}$ & 0.999 & $\begin{array}{l}\text { Damaging } \\
\text { (0) }\end{array}$ & $\begin{array}{l}\text { Disease- } \\
\text { causing }\end{array}$ & Gly & NO & Not found & $\begin{array}{l}\text { Likely pathogenic } \\
\text { (PM1,PM2,PP2,PP3) }\end{array}$ & YES \\
\hline p.(Gly1153Arg) & c. $3457 \mathrm{G}>\mathrm{C}$ & $3 \mathrm{M}, 2 \mathrm{~F}$ & 0.077 & Tol (0.515) & $\begin{array}{l}\text { Pol } \\
(0.9981)\end{array}$ & Gly & NO & Not found & VUS (PM1,PP2,BP4) & NO \\
\hline p.(Gly1303Cys) & c. $3907 \mathrm{G}>\mathrm{T}$ & $1 \mathrm{M}, 1 \mathrm{~F}$ & 1.00 & 0 & $\begin{array}{l}\text { Disease- } \\
\text { causing }\end{array}$ & Gly & NO & Not found & $\begin{array}{l}\text { Likely pathogenic } \\
\text { (PM1,PM2,PP2,PP3) }\end{array}$ & YES \\
\hline p.(Gly1330Ser) & c. $3988 \mathrm{G}>\mathrm{A}$ & $1 \mathrm{~F}$ & 0.998 & 0 & $\begin{array}{l}\text { Disease- } \\
\text { causing }\end{array}$ & Gly & NO & Not found & $\begin{array}{l}\text { Likely pathogenic } \\
\text { (PM1,PM2,PP2,PP3) }\end{array}$ & YES \\
\hline p.(Gly1339Ala) & c. $4016>C$ & $1 \mathrm{M}, 1 \mathrm{~F}$ & 1.00 & $\begin{array}{l}\text { Damaging } \\
(0.007)\end{array}$ & $\begin{array}{l}\text { Disease- } \\
\text { causing }\end{array}$ & Gly & NO & Not found & $\begin{array}{l}\text { Pathogenic } \\
\text { (PM1,PM2,PM5,PP2 } \\
\text {,PP3) }\end{array}$ & YES \\
\hline \multirow[t]{2}{*}{$\mathrm{GN}_{\mathrm{p} .(\mathrm{Gly} 1424 \mathrm{Ser})}$} & c. $4270 \mathrm{G}>\mathrm{A}$ & $3 F$ & $\begin{array}{l}\text { Probably } \\
\text { damaging } \\
0.998 \\
\end{array}$ & $\begin{array}{l}\text { Damaging } \\
\text { (0) }\end{array}$ & $\begin{array}{l}\text { Disease } \\
\text { causing } \\
(1.00)\end{array}$ & Gly & $\begin{array}{l}\text { LOVD, } \\
\text { p.(Gly1424Glu), } \\
\text { Zhang, 2011 }\end{array}$ & Not found & $\begin{array}{l}\text { Likely pathogenic } \\
\text { (PM1,PM2,PM5,PP2 } \\
\text {,PP3) }\end{array}$ & YES \\
\hline & & $\begin{array}{l}6 \mathrm{M}, 34 \mathrm{~F} \text { in } \\
83,712= \\
1 / 2093\end{array}$ & & & & & & & & \\
\hline
\end{tabular}

\section{$\mathrm{GN}$ also in gnomAD}


Suppl Table 8: Mitigating features for the clinical effects of predicted pathogenic COL4A5 variants resulting in position 1 Gly substitutions in gnomAD: associations with gender, location, and replacement residues

\begin{tabular}{|c|c|c|c|c|c|}
\hline Mutation & Alleles & Gender & Exons 1-20 & $\begin{array}{l}\text { Adjacent to non- } \\
\text { collagenous domain } \\
\text { or interruption }\end{array}$ & $\begin{array}{l}\text { Substitution } \\
\text { with Ala, Ser, } \\
\text { Cys }\end{array}$ \\
\hline p.(Gly69Arg) & 1 & $\mathrm{~F}$ & Yes & & \\
\hline p.(Gly84Glu) & 1 & $\mathrm{~F}$ & Yes & & \\
\hline p.(Gly105Ala) & 1 & $M$ & Yes & & Yes \\
\hline p.(Gly114Ala) & 1 & $\mathrm{~F}$ & Yes & & Yes \\
\hline p.(Gly126Glu) & 1 & $\mathrm{~F}$ & Yes & & \\
\hline p.(Gly201Ala) & 1 & $M$ & Yes & & Yes \\
\hline p.(Gly279Ala) & 1 & $M$ & Yes & & Yes \\
\hline p.(Gly283Val) & 1 & $\mathrm{~F}$ & Yes & Yes & \\
\hline p.(Gly286Cys) & 1 & $\mathrm{~F}$ & Yes & & Yes \\
\hline p.(Gly356Glu) & 1 & $M$ & Yes & Yes & \\
\hline p.(Gly356Ala) & 1 & $\mathrm{~F}$ & Yes & Yes & Yes \\
\hline p.(Gly512Glu)* & 1 & $M$ & & & \\
\hline p.(Gly536Ser) & 1 & $\mathrm{~F}$ & & & Yes \\
\hline p.(Gly624Asp) & 16 & $\begin{array}{l}M(n=4), F \\
(n=12)\end{array}$ & & Yes & \\
\hline p.(Gly656Ser) & 1 & $\mathrm{M}$ & & Yes & Yes \\
\hline p.(Gly702Ser) & 1 & $\mathrm{~F}$ & & & Yes \\
\hline p.(Gly752Val) & 2 & $M(n=2)$ & & Yes & \\
\hline p.(Gly787Ala) & 1 & $\mathrm{~F}$ & & & Yes \\
\hline p.(Gly822Glu)* & 1 & $M$ & & & \\
\hline p.(Gly893Val) & 1 & $\mathrm{~F}$ & & & \\
\hline p.(Gly967Arg) & 1 & $\mathrm{~F}$ & & & \\
\hline p.(Gly1054Asp)* & 1 & $M$ & & & \\
\hline p.(Gly1134Cys) & 1 & $\mathrm{~F}$ & & & Yes \\
\hline p.(Gly1170Ser) & 1 & $\mathrm{~F}$ & & & Yes \\
\hline p.(Gly1185Ser) & 1 & $\mathrm{~F}$ & & & Yes \\
\hline p.(Gly1249Arg) & 1 & $\mathrm{~F}$ & & Yes & \\
\hline p.(Gly1282Glu) & 1 & $M$ & & & \\
\hline p.(Gly1300Ala) & 1 & $\mathrm{~F}$ & & & Yes \\
\hline p.(Gly1303Ala) & 1 & $M$ & & & Yes \\
\hline p.(Gly1321Val) & 1 & $\mathrm{~F}$ & & & \\
\hline p.(Gly1324Glu) & 1 & $\mathrm{~F}$ & & & \\
\hline p.(Gly1333Cys) & 1 & $\mathrm{M}$ & & & Yes \\
\hline p.(Gly1394Cys) & 2 & $F(n=2)$ & & & Yes \\
\hline p.(Gly1424Ser) & 1 & $\mathrm{~F}$ & & & Yes \\
\hline $\mathrm{N}=34$ & 51 & $17 \mathrm{M}, 34 \mathrm{~F}$ & $\mathrm{~N}=11$ & $\mathrm{~N}=7$ & $\mathrm{~N}=18$ \\
\hline
\end{tabular}

$\mathrm{M}$ male, $\mathrm{F}$ female * variants where there were no biochemical features potentially mitigating clinical phenotype 
Suppl Table 9: Variants in COL4A3 and COL4A4 in gnomAD with inconsistent assessments subsequently excluded from population frequency studies

\begin{tabular}{|c|c|c|c|c|c|c|c|c|c|c|c|c|c|c|c|c|c|c|c|}
\hline hg19 & rs ID & Variant & $\begin{array}{l}\text { Trans } \\
\text { cript }\end{array}$ & PP2 & SIFT & MT & $\begin{array}{c}\text { Cons } \\
\text { erved in } \\
\text { verte- } \\
\text { brates } \\
\end{array}$ & $\begin{array}{l}\text { Clin } \\
\text { Var }\end{array}$ & Varsome & gnomad & $\begin{array}{l}\text { Cohort } \\
\text { size }\end{array}$ & A & $\mathbf{L}$ & AJ & EA & $\mathbf{F}$ & EUR & 0 & SA \\
\hline \multicolumn{20}{|l|}{ COLAA3 } \\
\hline 228102723 & rs13424243 & p.(Gly43Arg) & c. $127 \mathrm{G}>\mathrm{A}$ & 1 & DEL & $\mathbf{P}$ & Ser & Benign & VUS (PM1,PM2,PP2,BP4) & 3 & 280794 & 0 & 0 & 1 & 0 & 0 & 2 & 0 & 0 \\
\hline 228102723 & rs13424243 & p.(Gly43Arg) & c. $127 \mathrm{G}>\mathrm{C}$ & 1 & DEL & $\mathbf{P}$ & Ser & Benign & $\begin{array}{c}\text { Benign } \\
(\mathrm{PM} 1, \mathrm{PP} 2, \mathrm{BA} 1, \mathrm{BP} 4, \mathrm{BP} 6) \\
\end{array}$ & 80620 & 280794 & 7524 & 7749 & 3493 & 740 & 8390 & 46200 & 2242 & 4282 \\
\hline 228102723 & rs13424243 & p.(Gly43Trp) & $\begin{array}{l}c .127 \mathrm{G}>\mathrm{T} \\
\end{array}$ & 1 & DEL & $\mathbf{P}$ & Ser & Benign & VUS (PM1,PM2,PP2,BP4) & 80623 & 280794 & 1 & 0 & 0 & 0 & 0 & 1 & 0 & 0 \\
\hline 228102724 & rs776294835 & p.(Gly43Glu) & c. $128 \mathrm{G}>\mathrm{A}$ & 1 & DEL & $\mathbf{P}$ & Ser & Not found & VUS (PM1,PM2,PP2,BP4) & 3 & 249528 & 3 & 0 & 0 & 0 & 0 & 0 & 0 & 0 \\
\hline 228102732 & rs200866082 & p.(Gly46Arg) & c. $136 \mathrm{G}>\mathrm{A}$ & 1 & DEL & DC & Gly & Likely benign & $\begin{array}{c}\text { Likely pathogenic } \\
\text { (PM1,PM2,PP2,PP3,BP6) }\end{array}$ & 61 & 280882 & 57 & 3 & 0 & 0 & 0 & 0 & 1 & 0 \\
\hline 228131759 & rs745472969 & p.(Gly487Arg) & c. $1459 \mathrm{G}>\mathrm{C}$ & 1 & DEL & \begin{tabular}{|c|}
0.606 \\
$-P$
\end{tabular} & Gly & VUS & $\begin{array}{c}\text { Likely pathogenic } \\
\text { (PM1,PM2,PP2,PP3) }\end{array}$ & 1 & 249514 & 0 & 0 & 0 & 0 & 0 & 1 & 0 & 0 \\
\hline 228142243 & rs1331805495 & p.(Gly700Val) & c. $2099 \mathrm{G}>\mathrm{T}$ & 0.977 & \begin{tabular}{|c|} 
TOL \\
$(0.06)$ \\
\end{tabular} & DC & Gly & Not found & $\begin{array}{c}\text { Likely pathogenic } \\
\text { (PM1,PM2,PP2,PP3) }\end{array}$ & 1 & 245134 & 0 & 0 & 0 & 0 & 0 & 1 & 0 & 0 \\
\hline 228145668 & rs774838919 & p.(Gly812Ser) & c. $2434 \mathrm{G}>\mathrm{A}$ & 0.339 & $\begin{array}{c}\text { TOL } \\
(0.21) \\
\end{array}$ & $\mathbf{P}$ & Cys & Not found & $\begin{array}{c}\text { VUS (PM1, } \\
\text { PM2,PP2,BP4) }\end{array}$ & 10 & 178112 & 0 & 4 & 0 & 0 & 0 & 5 & 1 & 0 \\
\hline 228142227 & rs200287952 & p.(Gly695Arg) & c. $2083 \mathrm{G}>\mathrm{A}$ & 1 & $\begin{array}{l}\text { TOL } \\
0.08\end{array}$ & DC & Gly & Pathogenic & $\begin{array}{c}\text { Pathogenic } \\
\text { (PM1,PM2,PP2,PP3,PP5) }\end{array}$ & 31 & 278152 & 1 & 0 & 0 & 0 & 0 & 27 & 3 & 0 \\
\hline 228104886 & rs184730597 & p.(Gly58Ser) & c. $172 \mathrm{G}>\mathrm{A}$ & 1 & DEL & $\mathrm{DC}$ & Gly & vus & $\begin{array}{l}\text { Likely pathogenic } \\
\text { (PM1,PM2,PP2,PP3) }\end{array}$ & 6 & 243384 & 0 & 3 & 0 & 0 & 0 & 2 & 1 & 0 \\
\hline 228112275 & rs775373641 & p.(Gly148Val) & c. $443 \mathrm{G}>\mathrm{T}$ & 1 & DEL & DC & Gly & vUS & $\begin{array}{c}\text { Likely pathogenic } \\
\text { (PM1,PM2,PP2,PP3) }\end{array}$ & 4 & 280874 & 0 & 0 & 1 & 0 & 0 & 3 & 0 & 0 \\
\hline 228113159 & rs764451365 & p.(Gly157Arg) & c. $469 \mathrm{G}>\mathrm{C}$ & 1 & DEL & DC & Gly & vUS & $\begin{array}{c}\text { Likely pathogenic } \\
\text { (PM1,PM2,PP2,PP3) }\end{array}$ & 4 & 249350 & 0 & 0 & 0 & 0 & 0 & 1 & 1 & 2 \\
\hline 228131759 & rs745472969 & p.(Gly487Ser) & c. $1459 \mathrm{G}>\mathrm{A}$ & 1 & DEL & DC & Gly & vUS & $\begin{array}{c}\text { Likely pathogenic } \\
\text { (PM1,PM2,PM5,PP2,PP3) }\end{array}$ & 31 & 280912 & 0 & 15 & 0 & 0 & 0 & 10 & 1 & 5 \\
\hline 228149007 & rs1265432530 & p.(Gly943Arg) & c. $2827 \mathrm{G}>\mathrm{A}$ & 1 & DEL & DC & Gly & vUs & $\begin{array}{c}\text { Likely pathogenic } \\
\text { (PM1,PM2,PP2,PP3) }\end{array}$ & 2 & 249332 & 0 & 0 & 0 & 1 & 0 & 1 & 0 & 0 \\
\hline 228168602 & rs372237167 & p.(Gly1328Ala) & c. $3983 \mathrm{G}>\mathrm{C}$ & 1 & DEL & DC & Gly & vus & $\begin{array}{c}\text { Likely pathogenic } \\
\text { (PM1,PM2,PP2,PP3) }\end{array}$ & 1 & 249382 & 0 & 0 & 0 & 0 & 0 & 0 & 1 & 0 \\
\hline \multicolumn{20}{|l|}{ COL4A4 } \\
\hline 227984654 & rs1370340334 & p.(Gly110Ala) & c. $329 \mathrm{G}>\mathrm{C}$ & 1 & TOL & DC & Gly & Not found & $\begin{array}{c}\text { Likely pathogenic } \\
\text { (PM1,PM2, PP2,PP3) }\end{array}$ & 1 & 221310 & 0 & 0 & 0 & 0 & 0 & 1 & 0 & 0 \\
\hline 227984645 & rs766085522 & p.(Gly113Asp) & c. $338 \mathrm{G}>\mathrm{A}$ & 1 & TOL & DC & Gly & Not found & $\begin{array}{c}\text { Likely pathogenic } \\
\text { (PM1,PM2, PP2,PP3) }\end{array}$ & 14 & 257452 & 9 & 5 & 0 & 0 & 0 & 0 & 0 & 0 \\
\hline 227983440 & rs377511303 & p.(Gly137Asp) & c. $410 \mathrm{G}>\mathrm{A}$ & 1 & DEL & DC & Gly & VUS & $\begin{array}{c}\text { Likely pathogenic } \\
\text { (PM1,PM2, PP2,PP3) }\end{array}$ & 2 & 248802 & 2 & 0 & 0 & 0 & 0 & 0 & 0 & 0 \\
\hline 227983392 & rs773360119 & p.(Gly153Val) & $\begin{array}{lc}c .458 \mathrm{G}>\mathrm{T} \\
\end{array}$ & 0.712 & DEL & $\mathrm{DC}$ & Gly & Not found & $\operatorname{VUS}(\mathrm{PM} 1, \mathrm{PM} 2, \mathrm{PP} 2, \mathrm{BP} 4)$ & 2 & 249180 & 0 & 0 & 0 & 0 & 0 & 2 & 0 & 0 \\
\hline
\end{tabular}




\begin{tabular}{|c|c|c|c|c|c|c|c|c|c|c|c|c|c|c|c|c|c|c|c|}
\hline 227958987 & rs1026613471 & p.(Gly408Glu) & c.1223G>A & 1 & DEL & DC & Gly & $\begin{array}{c}\text { Conflicting; } \\
\text { Benign }\end{array}$ & $\begin{array}{l}\text { Likely pathogenic } \\
\text { (PM1,PM2,PP2,PP3) }\end{array}$ & 6 & 248574 & 0 & 0 & 5 & 0 & 0 & 1 & 0 & 0 \\
\hline 227954608 & rs202210475 & p.(Gly479Arg) & c. $1435 \mathrm{G}>\mathrm{C}$ & 0 & TOL & $\mathbf{P}$ & Gly & Conflcting;VUS & VUS (PM1,PM2,PP2,BP4) & 106 & 280814 & 0 & 6 & 75 & 0 & 0 & 16 & 8 & 1 \\
\hline 227946893 & rs 1800516 & p.(Gly545Ala) & $c .1634 \mathrm{G}>C$ & 1 & DEL & $\mathbf{P}$ & Gly & Benign & \begin{tabular}{|l|} 
Benign (PM1,PP2, \\
PP3,BS1,BS2,BP6) \\
\end{tabular} & 7694 & 280884 & 798 & 675 & 446 & 0 & 548 & 4724 & 262 & 241 \\
\hline 227924914 & rs781014928 & p.(Gly701Val) & c. $2102 \mathrm{G}>\mathrm{T}$ & 0.82 & TOL & DC & Gly & Not found & $\begin{array}{l}\text { Likely pathogenic } \\
\text { (PM1,PM2, PP2,PP3) }\end{array}$ & 1 & 248786 & 0 & 0 & 0 & 0 & 0 & 0 & 0 & 1 \\
\hline 227922308 & rs760803228 & p.(Gly798Ser) & c.2392G>A & 1 & DEL & DC & Gly & VUS & $\begin{array}{l}\text { Likely pathogenic } \\
\text { (PM1,PM2,PP2,PP3) }\end{array}$ & 3 & 280816 & 0 & 0 & 0 & 0 & 0 & 3 & 0 & 0 \\
\hline 227920820 & rs752296059 & p.(Gly853Arg) & c. $2557 \mathrm{G}>\mathrm{A}$ & 0 & TOL & $P$ & Gly & Not found & VUS (PM1,PM2,PP2,BP4) & 1 & 249324 & 0 & 0 & 0 & 1 & 0 & 0 & 0 & 0 \\
\hline 227915847 & rs13027659 & p.(Gly999Glu) & c.2996G>A & 1 & DEL & DC & Gly & $\begin{array}{c}\text { Conflicting; } \\
\text { benign; likely } \\
\text { benign }\end{array}$ & $\begin{array}{c}\text { VUS (PM1, PP2,PP3,PP5, } \\
\text { BS1,BS2) }\end{array}$ & 3321 & 280922 & 68 & 278 & 27 & 0 & 228 & 2508 & 104 & 108 \\
\hline 227915821 & rs371172166 & p.(Gly1008Arg) & c. $3022 \mathrm{G}>\mathrm{A}$ & 1 & DEL & DC & Gly & VUS & $\begin{array}{l}\text { Likely pathogenic } \\
\text { (PM1,PM2,PP2,PP3) }\end{array}$ & 9 & 280942 & 6 & 2 & 0 & 0 & 0 & 1 & 0 & 0 \\
\hline 227915799 & rs764323652 & p.(Gly1015Glu) & c.3044G>A & 1 & DEL & DC & Gly & $\begin{array}{c}\text { Conflicting; } \\
\text { Likely } \\
\text { pathogenic; VUS }\end{array}$ & $\begin{array}{l}\text { Likely pathogenic } \\
\text { (PM1,PM2,PP2,PP3) }\end{array}$ & 14 & 280944 & 0 & 0 & 0 & 0 & 0 & 14 & 0 & 0 \\
\hline 227915754 & rs772699709 & p.(Gly1030Val) & c. $3089 \mathrm{G}>\mathrm{T}$ & 1 & DEL & DC & Gly & vUS & $\begin{array}{l}\text { Likely pathogenic (PM1, } \\
\text { PM2,PM5,PP2,PP3,PP5) }\end{array}$ & 1 & 249562 & 0 & 0 & 0 & 0 & 0 & 1 & 0 & 0 \\
\hline 227983440 & rs377511303 & p.(Gly137Asp) & c. $410 \mathrm{G}>\mathrm{A}$ & 1 & DEL & DC & Gly & vUs & $\begin{array}{c}\text { Likely pathogenic } \\
\text { (PM1,PM2, PP2,PP3) }\end{array}$ & 2 & 248802 & 2 & 0 & 0 & 0 & 0 & 0 & 0 & 0 \\
\hline 227876903 & rs779769090 & p.(Gly1443Arg) & c. $4327 \mathrm{G}>\mathrm{A}$ & 0.005 & \begin{tabular}{|c|} 
DEL \\
$(0.22)$
\end{tabular} & $\mathbf{P}$ & Gly & Not found & VUS (PM1,PM2,PP2,BP4) & 1 & 249498 & 0 & 0 & 0 & 0 & 0 & 1 & 0 & 0 \\
\hline 227876903 & rs779769090 & p.(Gly1443Arg) & c. $4327 \mathrm{G}>\mathrm{C}$ & 0.005 & $\begin{array}{c}\text { DEL } \\
(0.22)\end{array}$ & $\mathbf{P}$ & Gly & Not found & VUS (PM1,PM2,PP2,BP4) & 13 & 249498 & 0 & 0 & 0 & 0 & 0 & 0 & 0 & 13 \\
\hline 227875175 & rs1287040507 & p.(Gly1459Val) & $c .4376 \mathrm{G}>\mathrm{T}$ & 0.627 & DEL & $\mathbf{P}$ & Gly & Not found & VUS (PM1,PM2,PP2,BP4) & 2 & 274180 & 0 & 0 & 0 & 0 & 0 & 2 & 0 & 0 \\
\hline
\end{tabular}

Evidence supporting pathogenicity is highlighted in red; and not supporting pathogenicity is in blue. PP2, Polyphen-2, SIFT, MT Mutation taster. PP2 scores $\mathbf{> 0 . 8}$ indicate Pathgoenic or Likely Pathogenic. SIFT scores DEL for deleterious, TOL for tolerated. MT scores DC for disease-causing and $P$ for polymorphism. Varsome online tool to assess ACMG criteria (varsome.com), gnomad variant database used to assess variant prevalence (approximate population $n=256,562$ for COL4A3, and 233,529 for COL4A4). Cohort size varied for different variants, A African ( $n=15,944)$ L Latino ( $n=32,544)$, AJ Ashenazi Jewish ( $n=9528)$, EA East Asian $(n=17,021)$, F Finnish ( $n=21,559)$, Eur European (non-Finnish, $n=111,786)$, Other $(n=6112)$, SE South Asian $(n=28,471)$

Of especial interest were p.(Gly545Ala) and p.(Gly999Glu) in COL4A4, which were not predicted pathogenic and excluded from further analysis. The p.(Gly545Ala) variant was found 7,626 times in gnomAD, including 145 times in the homozygous form. It was considered pathogenic in two out of three computational tools, affected a conserved residue, but had Benign assessments from ClinVar and Varsome (based on one moderate (PM1) and 2 supporting criteria for pathogenic (PP2, PP3) and two strong (BS1,BS2) and one supporting criteria for benign (BP6)). 
The p.(Gly999Glu) variant was found 3,259 times, including 33 times in the homozygous form. It was considered pathogenic in all three computational tools, and affected a conserved residue, but was considered a Variant of Uncertain Significance by both ClinVar and Varsome based on one moderate (PM1), and three supporting (PP2,PP3 and PP5) criteria for pathogenicity, and two strong criteria for benign (BS1 and BS2). 
Suppl Table 10: Predicted pathogenic founder variants in COL4A3 and COL4A4 in different ancestries in gnomAD

\begin{tabular}{|c|c|c|c|c|c|c|c|c|c|c|c|}
\hline Gene & Variant & Transcript & Total & African & Latino & Ashkenazi & $\begin{array}{l}\text { East } \\
\text { Asian }\end{array}$ & $\begin{array}{l}\text { Eur } \\
\text { (Finnish) }\end{array}$ & $\begin{array}{l}\text { Eur } \\
\text { (non- } \\
\text { Finnish) }\end{array}$ & Other & $\begin{array}{l}\text { South } \\
\text { Asian }\end{array}$ \\
\hline COL4A3 & None & & & & & & & & & & \\
\hline \multirow[t]{7}{*}{ COL4A4 } & p.(Gly445Ala) & c. $1334 \mathrm{G}>\mathrm{C}$ & 22 & 19 & & & & & 3 & & \\
\hline & p.(Gly481Ser) & c. $1441 \mathrm{G}>\mathrm{A}$ & 21 & & 21 & & & & & & \\
\hline & p.(Gly816Glu) & c. $2447 \mathrm{G}>\mathrm{A}$ & 24 & & & & 24 & & & & \\
\hline & p.(Gly1005Glu) & c. $3014 \mathrm{G}>\mathrm{A}$ & 25 & 1 & 23 & & & & 1 & & \\
\hline & p.(Gly1178Ser) & c. $3532 \mathrm{G}>\mathrm{A}$ & 24 & & 3 & & & 3 & 16 & 2 & \\
\hline & p.(Gly774Arg) & c. $2320 \mathrm{G}>\mathrm{C}$ & 19 & & & & & 1 & 5 & 13 & \\
\hline & p.(Ser969Ter) & c. $2906 C>G$ & 18 & 1 & & & & & 16 & 1 & \\
\hline
\end{tabular}

Variants were arbitrarily designated founder variants if they resulted in a position 1 Gly substitution, frameshift, nonsense or canonical splice variant that was predicted to be pathogenic and occurred $\geq 18$ times in gnomAD 
Suppl Table 11: Estimated population frequencies for predicted pathogenic heterozygous, compound heterozygous, and digenic variants

\begin{tabular}{|c|c|c|c|c|}
\hline COL4A3 & COL4A4 & Classification & Frequency & $\begin{array}{l}\text { Total population } \\
\text { frequency* }\end{array}$ \\
\hline$+/+$ & $+/+$ & Wildtype & $9.91 \times 10^{-1}$ & $\begin{array}{l}9.91 \times 10^{-1} \\
\text { or } 99 \text { in } 100 \\
\end{array}$ \\
\hline$+/+$ & $+/-$ & $\begin{array}{c}\text { Heterozygous } \\
\text { predicted pathogenic }\end{array}$ & $2.45 \times 10^{-3}$ & \multirow{4}{*}{ or one in 106} \\
\hline$+/+$ & $-/+$ & $\begin{array}{c}\text { Heterozygous } \\
\text { predicted pathogenic }\end{array}$ & $2.45 \times 10^{-3}$ & \\
\hline$+/-$ & $+/+$ & $\begin{array}{c}\text { Heterozygous } \\
\text { predicted pathogenic }\end{array}$ & $2.26 \times 10^{-3}$ & \\
\hline$-/+$ & $+/+$ & $\begin{array}{c}\text { Heterozygous } \\
\text { predicted pathogenic }\end{array}$ & $2.26 \times 10^{-3}$ & \\
\hline$+/-$ & $+/-$ & $\begin{array}{c}\text { Digenic predicted } \\
\text { pathogenic variants }\end{array}$ & $5.58 \times 10^{-6}$ & \multirow{4}{*}{$\begin{array}{l}2.23 \times 10^{-5} \\
\text { or one in } 44,793\end{array}$} \\
\hline$+/-$ & $-/+$ & $\begin{array}{c}\text { Digenic predicted } \\
\text { pathogenic variants }\end{array}$ & $5.58 \times 10^{-6}$ & \\
\hline$-/+$ & $+/-$ & $\begin{array}{c}\text { Digenic predicted } \\
\text { pathogenic variants }\end{array}$ & $5.58 \times 10^{-6}$ & \\
\hline$-/+$ & $-/+$ & $\begin{array}{c}\text { Digenic predicted } \\
\text { pathogenic variants }\end{array}$ & $5.58 \times 10^{-6}$ & \\
\hline$+/+$ & $-/-$ & $\begin{array}{l}\text { Compound } \\
\text { heterozygous predicted } \\
\text { pathogenic variants }\end{array}$ & $6.06 \times 10^{-6}$ & \multirow{7}{*}{$\begin{array}{c}1.13 \times 10^{-5} \\
\text { or one in } \\
88,866 \text { individuals }\end{array}$} \\
\hline$+/-$ & $-/-$ & $\begin{array}{l}\text { Compound } \\
\text { heterozygous predicted } \\
\text { pathogenic variants }\end{array}$ & $1.38 \times 10^{-8}$ & \\
\hline$-/+$ & $-/-$ & $\begin{array}{c}\text { Compound } \\
\text { heterozygous predicted } \\
\text { pathogenic variants }\end{array}$ & $1.38 \times 10^{-8}$ & \\
\hline$-/-$ & $+/+$ & $\begin{array}{c}\text { Compound } \\
\text { heterozygous predicted } \\
\text { pathogenic variants }\end{array}$ & $5.14 \times 10^{-6}$ & \\
\hline$-1-$ & $+/-$ & $\begin{array}{l}\text { Compound } \\
\text { heterozygous predicted } \\
\text { pathogenic variants }\end{array}$ & $1.27 \times 10^{-8}$ & \\
\hline$-/-$ & $-/+$ & $\begin{array}{c}\text { Compound } \\
\text { heterozygous predicted } \\
\text { pathogenic variants }\end{array}$ & $1.27 \times 10^{-8}$ & \\
\hline$-/-$ & $-/-$ & $\begin{array}{l}\text { Compound } \\
\text { heterozygous predicted } \\
\text { pathogenic variants }\end{array}$ & $3.14 \times 10^{-11}$ & \\
\hline
\end{tabular}

+ wildtype, - variant allele. The frequencies thave been calculated from the sum of the likelihood of the individual combinations of alleles. These calculations are based on the number of predicted pathogenic 
variants in the mean alleles examined that is 559/245,889 for COL4A3 and 577/233,916 for COL4A4 and assume that $\mathrm{COL} 4 A 3$ and $C O L 4 A 4$ variants occur together independently. If all predicted pathogenic variants are pathogenic, then heterozygous predicted pathogenic variants correspond to Thin basement membrane nephropathy or AD Alport syndrome; digenic predicted pathogenic variants correspond to digenic Alport syndrome; and compound heterozygous pathogenic variants correspond to the compound heterozygous form of AR Alport syndrome. It is not possible to use this method to calculate how commonly homozygous forms of predicted pathogenic variants occur because they are usually the result of consanguineous relationships and do not occur by chance. 


\section{References}

1. Gubler, M, Levy, M, Broyer, M, Naizot, C, Gonzales, G, Perrin, D, Habib, R: Alport's syndrome. A report of 58 cases and a review of the literature. Am J Med, 70: 493-505, 1981.

2. Barker, DF, Hostikka, SL, Zhou, J, Chow, LT, Oliphant, AR, Gerken, SC, Gregory, MC, Skolnick, MH, Atkin, CL, Tryggvason, K: Identification of mutations in the COL4A5 collagen gene in Alport syndrome. Science, 248: 1224-1227, 1990.

3. Mochizuki, T, Lemmink, HH, Mariyama, M, Antignac, C, Gubler, MC, Pirson, Y, Verellen-Dumoulin, C, Chan, B, Schroder, CH, Smeets, HJ, et al.: Identification of mutations in the alpha 3(IV) and alpha 4(IV) collagen genes in autosomal recessive Alport syndrome. Nat Genet, 8: 77-81, 1994.

4. Buzza, M, Wilson, D, Savige, J: Segregation of hematuria in thin basement membrane disease with haplotypes at the loci for Alport syndrome. Kidney Int, 59: 1670-1676, 2001.

5. Buzza, M, Dagher, H, Wang, YY, Wilson, D, Babon, JJ, Cotton, RG, Savige, J: Mutations in the COL4A4 gene in thin basement membrane disease. Kidney Int, 63: 447-453, 2003.

6. Savige, J: Should We Diagnose Autosomal Dominant Alport Syndrome When There Is a Pathogenic Heterozygous COL4A3 or COL4A4 Variant? Kidney Int Rep, 3: 1239-1241, 2018.

7. Savige, J, Rana, K, Tonna, S, Buzza, M, Dagher, H, Wang, YY: Thin basement membrane nephropathy. Kidney Int, 64: 1169-1178, 2003.

8. Mencarelli, MA, Heidet, L, Storey, H, van Geel, M, Knebelmann, B, Fallerini, C, Miglietti, N, Antonucci, MF, Cetta, F, Sayer, JA, van den Wijngaard, A, Yau, S, Mari, F, Bruttini, M, Ariani, F, Dahan, K, Smeets, B, Antignac, C, Flinter, F, Renieri, A: Evidence of digenic inheritance in Alport syndrome. $J$ Med Genet, 52: 163-174, 2015.

9. Voskarides, K, Damianou, L, Neocleous, V, Zouvani, I, Christodoulidou, S, Hadjiconstantinou, V, Ioannou, K, Athanasiou, Y, Patsias, C, Alexopoulos, E, Pierides, A, Kyriacou, K, Deltas, C: COL4A3/COL4A4 mutations producing focal segmental glomerulosclerosis and renal failure in thin basement membrane nephropathy. J Am Soc Nephrol, 18: 3004-3016, 2007.

10. Wickman, L, Hodgin, JB, Wang, SQ, Afshinnia, F, Kershaw, D, Wiggins, RC: Podocyte Depletion in Thin GBM and Alport Syndrome. PLoS One, 11: e0155255, 2016.

11. Baldovino, S, Moliner, AM, Taruscio, D, Daina, E, Roccatello, D: Rare Diseases in Europe: from a Wide to a Local Perspective. Isr Med Assoc J, 18: 359-363, 2016.

12. Hertz, JM, Thomassen, M, Storey, H, Flinter, F: Clinical utility gene card for: Alport syndrome - update 2014. Eur J Hum Genet, 23, 2015.

13. Hasstedt, SJ, Atkin, CL: X-linked inheritance of Alport syndrome: family P revisited. Am J Hum Genet, 35 : 1241-1251, 1983.

14. Persson, U, Hertz, JM, Wieslander, J, Segelmark, M: Alport syndrome in southern Sweden. Clin Nephrol, 64: 85-90, 2005.

15. Pajari, H, Kaariainen, H, Muhonen, T, Koskimies, O: Alport's syndrome in 78 patients: epidemiological and clinical study. Acta Paediatr, 85: 1300-1306, 1996.

16. Dodge, WF, West, EF, Smith, EH, Bruce, H, 3rd: Proteinuria and hematuria in schoolchildren: epidemiology and early natural history. J Pediatr, 88: 327-347, 1976.

17. Ritchie, CD, Bevan, EA, Collier, SJ: Importance of occult haematuria found at screening. Br Med J (Clin Res Ed), 292: 681-683, 1986.

18. Dische, FE, Anderson, VE, Keane, SJ, Taube, D, Bewick, M, Parsons, V: Incidence of thin membrane nephropathy: morphometric investigation of a population sample. J Clin Pathol, 43: 457-460, 1990.

19. Savige, J, Gregory, M, Gross, O, Kashtan, C, Ding, J, Flinter, F: Expert guidelines for the management of Alport syndrome and thin basement membrane nephropathy. J Am Soc Nephrol, 24: 364-375, 2013.

20. Jais, JP, Knebelmann, B, Giatras, I, De Marchi, M, Rizzoni, G, Renieri, A, Weber, M, Gross, O, Netzer, KO, Flinter, F, Pirson, Y, Verellen, C, Wieslander, J, Persson, U, Tryggvason, K, Martin, P, Hertz, JM, Schroder, C, Sanak, M, Krejcova, S, Carvalho, MF, Saus, J, Antignac, C, Smeets, H, Gubler, MC: Xlinked Alport syndrome: natural history in 195 families and genotype- phenotype correlations in males. J Am Soc Nephrol, 11: 649-657, 2000.

21. Jais, JP, Knebelmann, B, Giatras, I, De Marchi, M, Rizzoni, G, Renieri, A, Weber, M, Gross, O, Netzer, KO, Flinter, F, Pirson, Y, Dahan, K, Wieslander, J, Persson, U, Tryggvason, K, Martin, P, Hertz, JM, Schroder, C, Sanak, M, Carvalho, MF, Saus, J, Antignac, C, Smeets, H, Gubler, MC: X-linked Alport syndrome: natural history and genotype-phenotype correlations in girls and women belonging to 195 families: a "European Community Alport Syndrome Concerted Action" study. J Am Soc Nephrol, 14: 2603-2610, 2003.

22. Savige, J, Colville, D, Rheault, M, Gear, S, Lennon, R, Lagas, S, Finlay, M, Flinter, F: Alport Syndrome in Women and Girls. Clin J Am Soc Nephrol, 11: 1713-1720, 2016. 
23. Savige, J, Ariani, F, Mari, F, Bruttini, M, Renieri, A, Gross, O, Deltas, C, Flinter, F, Ding, J, Gale, DP, Nagel, M, Yau, M, Shagam, L, Torra, R, Ars, E, Hoefele, J, Garosi, G, Storey, H: Expert consensus guidelines for the genetic diagnosis of Alport syndrome. Pediatr Nephrol, 2018.

24. Richards, S, Aziz, N, Bale, S, Bick, D, Das, S, Gastier-Foster, J, Grody, WW, Hegde, M, Lyon, E, Spector, E, Voelkerding, K, Rehm, HL, Committee, ALQA: Standards and guidelines for the interpretation of sequence variants: a joint consensus recommendation of the American College of Medical Genetics and Genomics and the Association for Molecular Pathology. Genet Med, 17: 405-424, 2015.

25. Myllyharju, J, Kivirikko, KI: Collagens and collagen-related diseases. Ann Med, 33: 7-21, 2001.

26. Mariyama, M, Leinonen, A, Mochizuki, T, Tryggvason, K, Reeders, ST: Complete primary structure of the human alpha 3(IV) collagen chain. Coexpression of the alpha 3(IV) and alpha 4(IV) collagen chains in human tissues. J Biol Chem, 269: 23013-23017, 1994.

27. Leinonen, A, Mariyama, M, Mochizuki, T, Tryggvason, K, Reeders, ST: Complete primary structure of the human type IV collagen alpha 4(IV) chain. Comparison with structure and expression of the other alpha (IV) chains. J Biol Chem, 269: 26172-26177, 1994.

28. Zhou, J, Hertz, JM, Leinonen, A, Tryggvason, K: Complete amino acid sequence of the human alpha 5 (IV) collagen chain and identification of a single-base mutation in exon 23 converting glycine 521 in the collagenous domain to cysteine in an Alport syndrome patient. J Biol Chem, 267: 12475-12481, 1992.

29. Demosthenous, P, Voskarides, K, Stylianou, K, Hadjigavriel, M, Arsali, M, Patsias, C, Georgaki, E, Zirogiannis, P, Stavrou, C, Daphnis, E, Pierides, A, Deltas, C, Hellenic Nephrogenetics Research, C: $\mathrm{X}$-linked Alport syndrome in Hellenic families: phenotypic heterogeneity and mutations near interruptions of the collagen domain in COL4A5. Clin Genet, 81: 240-248, 2012.

30. Lanktree, MB, Haghighi, A, Guiard, E, Iliuta, IA, Song, X, Harris, PC, Paterson, AD, Pei, Y: Prevalence Estimates of Polycystic Kidney and Liver Disease by Population Sequencing. J Am Soc Nephrol, 29: 2593-2600, 2018.

31. Lek, M, Karczewski, KJ, Minikel, EV, Samocha, KE, Banks, E, Fennell, T, O'Donnell-Luria, AH, Ware, JS, Hill, AJ, Cummings, BB, Tukiainen, T, Birnbaum, DP, Kosmicki, JA, Duncan, LE, Estrada, K, Zhao, F, Zou, J, Pierce-Hoffman, E, Berghout, J, Cooper, DN, Deflaux, N, DePristo, M, Do, R, Flannick, J, Fromer, M, Gauthier, L, Goldstein, J, Gupta, N, Howrigan, D, Kiezun, A, Kurki, MI, Moonshine, AL, Natarajan, P, Orozco, L, Peloso, GM, Poplin, R, Rivas, MA, Ruano-Rubio, V, Rose, SA, Ruderfer, DM, Shakir, K, Stenson, PD, Stevens, C, Thomas, BP, Tiao, G, Tusie-Luna, MT, Weisburd, B, Won, HH, Yu, D, Altshuler, DM, Ardissino, D, Boehnke, M, Danesh, J, Donnelly, S, Elosua, R, Florez, JC, Gabriel, SB, Getz, G, Glatt, SJ, Hultman, CM, Kathiresan, S, Laakso, M, McCarroll, S, McCarthy, MI, McGovern, D, McPherson, R, Neale, BM, Palotie, A, Purcell, SM, Saleheen, D, Scharf, JM, Sklar, P, Sullivan, PF, Tuomilehto, J, Tsuang, MT, Watkins, HC, Wilson, JG, Daly, MJ, MacArthur, DG, Exome Aggregation, C: Analysis of protein-coding genetic variation in 60,706 humans. Nature, 536: 285-291, 2016.

32. The National Genomics Research and Healthcare Knowledgebase v5, Genomics England. 5, 2019.

33. van der Ven, AT, Connaughton, DM, Ityel, H, Mann, N, Nakayama, M, Chen, J, Vivante, A, Hwang, DY, Schulz, J, Braun, DA, Schmidt, JM, Schapiro, D, Schneider, R, Warejko, JK, Daga, A, Majmundar, AJ, Tan, W, Jobst-Schwan, T, Hermle, T, Widmeier, E, Ashraf, S, Amar, A, Hoogstraaten, CA, Hugo, H, Kitzler, TM, Kause, F, Kolvenbach, CM, Dai, R, Spaneas, L, Amann, K, Stein, DR, Baum, MA, Somers, MJG, Rodig, NM, Ferguson, MA, Traum, AZ, Daouk, GH, Bogdanovic, R, Stajic, N, Soliman, NA, Kari, JA, El Desoky, S, Fathy, HM, Milosevic, D, Al-Saffar, M, Awad, HS, Eid, LA, Selvin, A, Senguttuvan, P, Sanna-Cherchi, S, Rehm, HL, MacArthur, DG, Lek, M, Laricchia, KM, Wilson, MW, Mane, SM, Lifton, RP, Lee, RS, Bauer, SB, Lu, W, Reutter, HM, Tasic, V, Shril, S, Hildebrandt, F: Whole-Exome Sequencing Identifies Causative Mutations in Families with Congenital Anomalies of the Kidney and Urinary Tract. J Am Soc Nephrol, 29: 2348-2361, 2018.

34. Biesecker, LG, Harrison, SM, ClinGen Sequence Variant Interpretation Working, G: The ACMG/AMP reputable source criteria for the interpretation of sequence variants. Genet Med, 20: 1687-1688, 2018.

35. Richards, CS, Aziz, N, Bale, S, Bick, D, Das, S, Gastier-Foster, J, Grody, WW, Hegde, M, Lyon, E, Spector, E, Voelkerding, K, Rehm, HL, Group, AAIoSVW: Response to Biesecker and Harrison. Genet Med, 20: 1689-1690, 2018.

36. Abou Tayoun, AN, Pesaran, T, DiStefano, MT, Oza, A, Rehm, HL, Biesecker, LG, Harrison, SM, ClinGen Sequence Variant Interpretation Working, G: Recommendations for interpreting the loss of function PVS1 ACMG/AMP variant criterion. Hum Mutat, 39: 1517-1524, 2018.

37. Hashimura, Y, Nozu, K, Kaito, H, Nakanishi, K, Fu, XJ, Ohtsubo, H, Hashimoto, F, Oka, M, Ninchoji, T, Ishimori, S, Morisada, N, Matsunoshita, N, Kamiyoshi, N, Yoshikawa, N, Iijima, K: Milder clinical aspects of X-linked Alport syndrome in men positive for the collagen IV alpha5 chain. Kidney Int, 85: 1208-1213, 2014. 
38. Savige, J, Storey, H, Il Cheong, H, Gyung Kang, H, Park, E, Hilbert, P, Persikov, A, Torres-Fernandez, C, Ars, E, Torra, R, Hertz, JM, Thomassen, M, Shagam, L, Wang, D, Wang, Y, Flinter, F, Nagel, M: XLinked and Autosomal Recessive Alport Syndrome: Pathogenic Variant Features and Further Genotype-Phenotype Correlations. PLoS One, 11: e0161802, 2016.

39. Persikov, AV, Pillitteri, RJ, Amin, P, Schwarze, U, Byers, PH, Brodsky, B: Stability related bias in residues replacing glycines within the collagen triple helix (Gly-Xaa-Yaa) in inherited connective tissue disorders. Hum Mutat, 24: 330-337, 2004.

40. Storey, H, Savige, J, Sivakumar, V, Abbs, S, Flinter, FA: COL4A3/COL4A4 mutations and features in individuals with autosomal recessive Alport syndrome. J Am Soc Nephrol, 24: 1945-1954, 2013.

41. Lennon, R, Stuart, HM, Bierzynska, A, Randles, MJ, Kerr, B, Hillman, KA, Batra, G, Campbell, J, Storey, H, Flinter, FA, Koziell, A, Welsh, GI, Saleem, MA, Webb, NJ, Woolf, AS: Coinheritance of COL4A5 and MYO1E mutations accentuate the severity of kidney disease. Pediatr Nephrol, 30: 1459-1465, 2015.

42. Knebelmann, B, Breillat, C, Forestier, L, Arrondel, C, Jacassier, D, Giatras, I, Drouot, L, Deschenes, G, Grunfeld, JP, Broyer, M, Gubler, MC, Antignac, C: Spectrum of mutations in the COL4A5 collagen gene in X-linked Alport syndrome. Am J Hum Genet, 59: 1221-1232, 1996.

43. Inoue, YJ, Nishio, H, Shirakawa, T, Nakanishi, K, Nakamura, H, Sumino, K, Nishiyama, K, Iijima, K, Yoshikawa, N: Detection of mutations in the COL4A5 gene in over $90 \%$ of male patients with Xlinked Alport's syndrome by RT-PCR and direct sequencing. Am J Kidney Dis, 34: 854-862, 1999.

44. Zhang, Y, Ding, J, Wang, S, Zhang, H, Zhong, X, Liu, X, Xu, K, Wang, F: Reassessing the pathogenicity of c.2858G>T(p.(G953V)) in COL4A5 Gene: report of 19 Chinese families. Eur J Hum Genet, 28: 244252, 2020.

45. Slajpah, M, Gorinsek, B, Berginc, G, Vizjak, A, Ferluga, D, Hvala, A, Meglic, A, Jaksa, I, Furlan, P, Gregoric, A, Kaplan-Pavlovcic, S, Ravnik-Glavac, M, Glavac, D: Sixteen novel mutations identified in COL4A3, COL4A4, and COL4A5 genes in Slovenian families with Alport syndrome and benign familial hematuria. Kidney Int, 71: 1287-1295, 2007.

46. Macheroux, EP, Braunisch, MC, Pucci Pegler, S, Satanovskij, R, Riedhammer, KM, Gunthner, R, Gross, O, Nagel, M, Renders, L, Hoefele, J: The Hypomorphic Variant p.(Gly624Asp) in COL4A5 as a Possible Cause for an Unexpected Severe Phenotype in a Family With X-Linked Alport Syndrome. Front Pediatr, 7: 485, 2019.

47. Zurowska, AM, Bielska, O, Daca-Roszak, P, Jankowski, M, Szczepanska, M, Roszkowska-Bjanid, D, Kuzma-Mroczkowska, E, Panczyk-Tomaszewska, M, Moczulska, A, Drozdz, D, Hadjipanagi, D, Deltas, C, Ostalska-Nowicka, D, Rabiega, A, Taraszkiewicz, J, Taranta-Janusz, K, WieczorkiewiczPlaza, A, Jobs, K, Mews, J, Musial, K, Jakubowska, A, Nosek, H, Jander, AE, Koutsofti, C, Stanislawska-Sachadyn, A, Kuleszo, D, Zietkiewicz, E, Lipska-Zietkiewicz, BS: Mild X-linked Alport syndrome due to the COL4A5 G624D variant originating in the Middle Ages is predominant in Central/East Europe and causes kidney failure in midlife. Kidney Int, 2020.

48. AM, Z: Mild X-linked Alport syndrome due to the COL4A5 G624D variant origtinating in the Middle Ages is predominant in Central/East Europe and causes kidney failure in mid-life. Kidney Int, 2021.

49. Persikov, AV, Ramshaw, JA, Kirkpatrick, A, Brodsky, B: Amino acid propensities for the collagen triplehelix. Biochemistry, 39: 14960-14967, 2000.

50. Fallerini, C, Baldassarri, M, Trevisson, E, Morbidoni, V, La Manna, A, Lazzarin, R, Pasini, A, Barbano, G, Pinciaroli, AR, Garosi, G, Frullanti, E, Pinto, AM, Mencarelli, MA, Mari, F, Renieri, A, Ariani, F: Alport syndrome: impact of digenic inheritance in patients management. Clin Genet, 92: 34-44, 2017.

51. Feingold, J, Bois, E, Chompret, A, Broyer, M, Gubler, MC, Grunfeld, JP: Genetic heterogeneity of Alport syndrome. Kidney Int, 27: 672-677, 1985.

52. Lemmink, HH, Nillesen, WN, Mochizuki, T, Schroder, CH, Brunner, HG, van Oost, BA, Monnens, LA, Smeets, HJ: Benign familial hematuria due to mutation of the type IV collagen alpha4 gene. J Clin Invest, 98: 1114-1118, 1996.

53. Makareeva, E, Mertz, EL, Kuznetsova, NV, Sutter, MB, DeRidder, AM, Cabral, WA, Barnes, AM, McBride, DJ, Marini, JC, Leikin, S: Structural heterogeneity of type I collagen triple helix and its role in osteogenesis imperfecta. J Biol Chem, 283: 4787-4798, 2008.

54. Gross, O, Licht, C, Anders, HJ, Hoppe, B, Beck, B, Tonshoff, B, Hocker, B, Wygoda, S, Ehrich, JH, Pape, L, Konrad, M, Rascher, W, Dotsch, J, Muller-Wiefel, DE, Hoyer, P, Study Group Members of the Gesellschaft fur Padiatrische, N, Knebelmann, B, Pirson, Y, Grunfeld, JP, Niaudet, P, Cochat, P, Heidet, L, Lebbah, S, Torra, R, Friede, T, Lange, K, Muller, GA, Weber, M: Early angiotensinconverting enzyme inhibition in Alport syndrome delays renal failure and improves life expectancy. Kidney Int, 81: 494-501, 2012.

55. Kashtan, CE: Alport Syndrome. Gene Reviews 
In: Adam MP, Ardinger HH, Pagon RA, et al., editors. GeneReviews® [Internet]. Seattle (WA): University of Washington, Seattle; 1993-2020. Available from: https://www.ncbi.nlm.nih.gov/books/NBK1207/. 2001.

56. Pierides, A, Voskarides, K, Athanasiou, Y, Ioannou, K, Damianou, L, Arsali, M, Zavros, M, Pierides, M, Vargemezis, V, Patsias, C, Zouvani, I, Elia, A, Kyriacou, K, Deltas, C: Clinico-pathological correlations in 127 patients in 11 large pedigrees, segregating one of three heterozygous mutations in the COL4A3/ COL4A4 genes associated with familial haematuria and significant late progression to proteinuria and chronic kidney disease from focal segmental glomerulosclerosis. Nephrol Dial Transplant, 24: 2721-2729, 2009.

57. Matthaiou, M, Poulii, T, Matthaiou, M, Matthaiou, M, Poulli, T, Deltas, C: Prevalence of clinical, pathological and molecular features of glomerular basement membrane nephropathy caused by COLAA3 or COLAA4 mutations: a systematic review. Clinical Kidney Journal 1-12, 2020.

58. Gillion, V, Dahan, K, Cosyns, JP, Hilbert, P, Jadoul, M, Goffin, E, Godefroid, N, De Meyer, M, Mourad, M, Pirson, Y, Kanaan, N: Genotype and Outcome After Kidney Transplantation in Alport Syndrome. Kidney Int Rep, 3: 652-660, 2018.

59. Groopman, EE, Marasa, M, Cameron-Christie, S, Petrovski, S, Aggarwal, VS, Milo-Rasouly, H, Li, Y, Zhang, J, Nestor, J, Krithivasan, P, Lam, WY, Mitrotti, A, Piva, S, Kil, BH, Chatterjee, D, Reingold, R, Bradbury, D, DiVecchia, M, Snyder, H, Mu, X, Mehl, K, Balderes, O, Fasel, DA, Weng, C, Radhakrishnan, J, Canetta, P, Appel, GB, Bomback, AS, Ahn, W, Uy, NS, Alam, S, Cohen, DJ, Crew, RJ, Dube, GK, Rao, MK, Kamalakaran, S, Copeland, B, Ren, Z, Bridgers, J, Malone, CD, Mebane, CM, Dagaonkar, N, Fellstrom, BC, Haefliger, C, Mohan, S, Sanna-Cherchi, S, Kiryluk, K, Fleckner, J, March, R, Platt, A, Goldstein, DB, Gharavi, AG: Diagnostic Utility of Exome Sequencing for Kidney Disease. N Engl J Med, 380: 142-151, 2019.

60. Fallerini, C, Dosa, L, Tita, R, Del Prete, D, Feriozzi, S, Gai, G, Clementi, M, La Manna, A, Miglietti, N, Mancini, R, Mandrile, G, Ghiggeri, GM, Piaggio, G, Brancati, F, Diano, L, Frate, E, Pinciaroli, AR, Giani, M, Castorina, P, Bresin, E, Giachino, D, De Marchi, M, Mari, F, Bruttini, M, Renieri, A, Ariani, F: Unbiased next generation sequencing analysis confirms the existence of autosomal dominant Alport syndrome in a relevant fraction of cases. Clin Genet, 86: 252-257, 2014.

61. Bullich, G, Domingo-Gallego, A, Vargas, I, Ruiz, P, Lorente-Grandoso, L, Furlano, M, Fraga, G, Madrid, A, Ariceta, G, Borregan, M, Pinero-Fernandez, JA, Rodriguez-Pena, L, Ballesta-Martinez, MJ, LlanoRivas, I, Menica, MA, Ballarin, J, Torrents, D, Torra, R, Ars, E: A kidney-disease gene panel allows a comprehensive genetic diagnosis of cystic and glomerular inherited kidney diseases. Kidney Int, 94: 363-371, 2018.

62. Gross, O, Tonshoff, B, Weber, LT, Pape, L, Latta, K, Fehrenbach, H, Lange-Sperandio, B, Zappel, H, Hoyer, P, Staude, H, Konig, S, John, U, Gellermann, J, Hoppe, B, Galiano, M, Hoecker, B, Ehren, R, Lerch, C, Kashtan, CE, Harden, M, Boeckhaus, J, Friede, T, German Pediatric Nephrology Study, G, Investigators, EP-TA: A multicenter, randomized, placebo-controlled, double-blind phase 3 trial with open-arm comparison indicates safety and efficacy of nephroprotective therapy with ramipril in children with Alport's syndrome. Kidney Int, 97: 1275-1286, 2020.

63. Hertz, JM, Juncker, I, Persson, U, Matthijs, G, Schmidtke, J, Petersen, MB, Kjeldsen, M, Gregersen, N: Detection of mutations in the COL4A5 gene by SSCP in X-linked Alport syndrome. Hum Mutat, 18: 141-148, 2001.

64. Plant, KE, Green, PM, Vetrie, D, Flinter, FA: Detection of mutations in COL4A5 in patients with Alport syndrome. Hum Mutat, 13: 124-132, 1999.

65. King, K, Flinter, FA, Green, PM: A two-tier approach to mutation detection in the COL4A5 gene for Alport syndrome. Hum Mutat, 27: 1061, 2006.

66. Cruz-Robles, D, Garcia-Torres, R, Antignac, C, Forestier, L, de la Puente, SG, Correa-Rotter, R, GarciaLopez, E, Orozco, L: Three novel mutations in the COL4A5 gene in Mexican Alport syndrome patients. Clin Genet, 56: 242-243, 1999.

67. Bekheirnia, MR, Reed, B, Gregory, MC, McFann, K, Shamshirsaz, AA, Masoumi, A, Schrier, RW: Genotype-phenotype correlation in X-linked Alport syndrome. J Am Soc Nephrol, 21: 876-883, 2010.

68. Mohammad, M, Nanra, R, Colville, D, Trevillian, P, Wang, Y, Storey, H, Flinter, F, Savige, J: A female with X-linked Alport syndrome and compound heterozygous COL4A5 mutations. Pediatr Nephrol, 29: 481-485, 2014.

69. Inoue, Y, Nishio, H, Shirakawa, T, Nakanishi, K, Nakamura, H, Sumino, K, Nishiyama, K, Iijima, K, Yoshikawa, N: Detection of mutations in the COL4A5 gene in over $90 \%$ of male patients with Xlinked Alport's syndrome by RT-PCR and direct sequencing. Am J Kidney Dis, 34: 854-862, 1999.

70. Zhang, H, Ding, J, Wang, F, Zhao, D: Mutation detection of COL4An gene based on mRNA of peripheral blood lymphocytes and prenatal diagnosis of Alport syndrome in China. Nephrology (Carlton), 16: 377-380, 2011. 
